$$
N p=-4901749
$$

\title{
MINERAL REVENUES: THE 1983 REPORT ON RECEIPTS FROM FEDERAL AND INDIAN LEASES
}

WITH SUMMARY DATA FROM 1920 TO 1983

\section{NOTICE}

PORTIONS OF THIS REPORT ARE ILLEGIBLE. II fias been reproduced from the best available copy to permit the broadest pessible avail. ability.
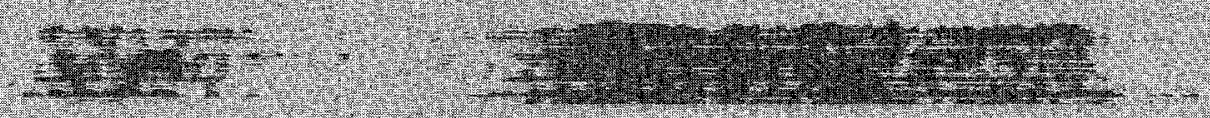

$$
\mathrm{NP}-4901749
$$

TI84 901749 


\section{DISCLAIMER}

This report was prepared as an account of work sponsored by an agency of the United States Government. Neither the United States Government nor any agency Thereof, nor any of their employees, makes any warranty, express or implied, or assumes any legal liability or responsibility for the accuracy, completeness, or usefulness of any information, apparatus, product, or process disclosed, or represents that its use would not infringe privately owned rights. Reference herein to any specific commercial product, process, or service by trade name, trademark, manufacturer, or otherwise does not necessarily constitute or imply its endorsement, recommendation, or favoring by the United States Government or any agency thereof. The views and opinions of authors expressed herein do not necessarily state or reflect those of the United States Government or any agency thereof. 


\section{DISCLAIMER}

Portions of this document may be illegible in electronic image products. Images are produced from the best available original document. 


\section{U.S. DEPARTMENT OF THE INTERIOR WILLIAM P. CLARK, Secretary}

MINERALS MANAGEMENT SERVICE William D. Bettenberg, Director

\section{ACKNOWLEDGMENTS:}

This report was prepared with the assistance of Walter Harris, Marilyn Johnson, and Bruce McFarlane; and edited and designed by Patrice La Liberté-all of the Royalty Management Program, Minerals Management Service.

Special thanks are also due to Jane Timmins and Susan Kingsbury, of the U.S. Geological Survey, and to Donal Ziehl, of the Bureau of Land Management.

Note: Some information in this report necessarily includes estimated data that may either be revised or corrected subsequent to publication.

U.S. Government Printing Office / 1984

Washington, D.C. 20402 


\section{CONTENTS}

Foreword vii

\section{MINERAL REVENUE MANAGEMENT IN 19831}

Figure 1:

Royalties and revenues other than bonuses in millions from Federal and Indian mineral leases, 1979-83 I

Table 1-a:

1983 mineral revenues from Federal and Indian mineral leases in the United States 2

Table 1-be

1983 disbursements of mineral revenues from Federal and Indian mineral leases in the United States 3

Table 2.

Summary of royalty revenues collected from Federal and Indian mineral leases in the United States, 1920-83 4

Figure 2

Royalties and revenues other than bonuses in millions from Federal and Indian mineral leases, 1920-90 5

Table 3:

1983 volume, value, royalties and other revenues from selected solid minerals other than coal from Federal and Indian leases 6

Table 4:

Summary of volume, value, royalties and other revenues from selected minerals from Federal and Indian leases, 1920-83 6

\section{OFFSHORE FEDERAL MINERAL REVENUES 8}

Figure 3

Royalties and revenues other than bonuses in millions from offshore Federal mineral leases, 1979-83 8

Table 5:

1983 volume, value, royalties and other revenues from offshore Federal mineral leases by OCS area and commodity 10 


\section{OFFSHORE FEDERAL MINERAL REVENUES (cont'd):}

Table 6:

Summary of total volume, value, royalties, and other revenues from offshore Federal mineral leases by OCS area and commodity, 1953-83 11

Table 7:

Summary of volume, value, royalties and other revenues from offshore Federal mineral leases by OCS area and year, 1953-83 12

Figure 4:

1983 offshore royalties and other mineral revenues as percentages of total Federal and Indian collections 14

\section{Table 8:}

Summary of all volume, value, royalties and other revenues from all offshore Federal mineral leases, 1953-83 15

Table 9:

1983 bonuses paid on sales of offshore Federal oil \& gas leases by OCS areas 16

Table 10:

Summary of bonuses paid on sales of offshore Federal oil \& gas leases, 1954-83 16

\section{ONSHORE FEDERAL MINERAL REVENUES 18}

Figure 5

Royalties and revenues other than bonuses in millions from onshore Federal mineral leases, 1979-83 18

Figure 6:

1983 onshore royalties and other revenues as percentages of total Federal and Indian collections from oil, gas, and coal 19

Table 11:

Summary of volume, value, royalties and other revenues from onshore Federal mineral leases by State and commodity, 1920-83 20

Table 12:

1983 bonuses paid on sales of onshore Federal mineral leases by State and commodity 35

Table 13:

Summary of bonuses paid on sales of onshore Federal mineral leases, 1954-83 36 


\section{INDIAN MINERAL REVENUES 38}

Figure 7:

Indian royalties and revenues other than bonuses in millions from Indian mineral leases, $1979-8338$

\section{List:}

Indian Tribes, Nations, and Councils with mineral leases producing in 198339

Table 14:

Summary of volume, value, royalties and other revenues from Indian mineral leases by State and commodity, 1937-83 40

\section{DISTRIBUTION OF FEDERAL AND INDIAN MINERAL REVENUES, 48}

Figure 8:

1983 disbursement of mineral revenues in millions from Federal and Indian mineral leases 48

\section{Figure $9:$}

1983 distribution to States of $\$ 454.4$ million share in royalties and other revenues from Federal mineral leases 49

Table 15:

1983 royalties, rents, and bonuses from mineral leases in Federal and Indian lands 51

Table 16:

Summary of distribution of mineral revenue shares to the 26 States in which Federal mineral leases are located, 1920-83 52

Table 17:

Summary of Indian royalties and other mineral receipts by year and commodity, 1937-83 53

Figure 10:

Royalties and revenues other than bonuses in millions by fiscal year from Federal and Indian mineral leases, 1979-83 53

\section{APPENDIX: LEASE MANAGEMENT DATA, 57}

Table 18:

Summary of royalty-in-kind oil taken as royalty payments for Federal lease production, 1976-83 57 


\section{APPENDIX: LEASE MANAGEMENT DATA (cont'd):}

Table 19:

Producing and producible Federal and Indian oil \& gas leases onshore in 26 States and offshore in 4 OCS areas, as of December 31, 198358

Table 20:

Producing Federal and Indian coal leases in 10 States, as of December 31, 1983 59

Table 21:

Producing Federal and Indian leases of other minerals in 12 States, as of December 31, 198360

Table 22:

Summary of oil \& gas and other mineral leases, licenses, and permits under supervision of the Department of the Interior in the of fshore Federal lands, as of December 31, 198361

Table 23:

Summary of oil \& gas and other mineral leases, contracts, licenses, and permits under the supervision of the Department of the Interior in the Federal and Indian onshore lands, as of December 31, 198362

Table 24:

Summary of the general terms in Federal and Indian mineral leases 64

Table 25:

Summary of oil, gas, and coal production from Federal and Indian leases as a percentage of total U.S. production of oil, gas, and coal, 1950-83 68 


\section{$x$ \\ FOREWORD}

For the third year the Minerals Management Service is pleased to bring you Mineral Revenues, a report on revenues collected and distributed from Federal and Indian mineral leases.

As the pages of our publication clearly show, mineral revenues from Federal and Indian lands are subject to the same economic vagaries that affect the whole minerals extraction industry. While overall revenues increased by more than $\$ 1$ billion from 1982 , royalty collections were reduced in 1983 by about $\$ 452$ million, reflecting modest declines in oil prices and somewhat sharper declines in gas production.

The highlight of 1983 was the accomplishment of the first areawide sales under the 5-Year OCS Leasing Program. The highly successful Gulf of Mexico offerings accounted for a substantial increase in OCS bonus receipts.

The renovation begun in October 1980 of the Royalty Management Program continues in the Minerals Management Service. Each month measurable progress is made toward our goal of assuring the American public that all mineral revenues due and owing from Federal and Indian lands have been properly collected, accounted for, and distributed to the recipients designated by law. It is a large job; a job requiring continued dedication on the part of the more than $500 \mathrm{em}$ ployees who now make up the Royalty Management Program.

William D. Bettenberg

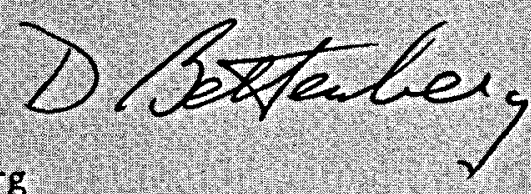

Director 


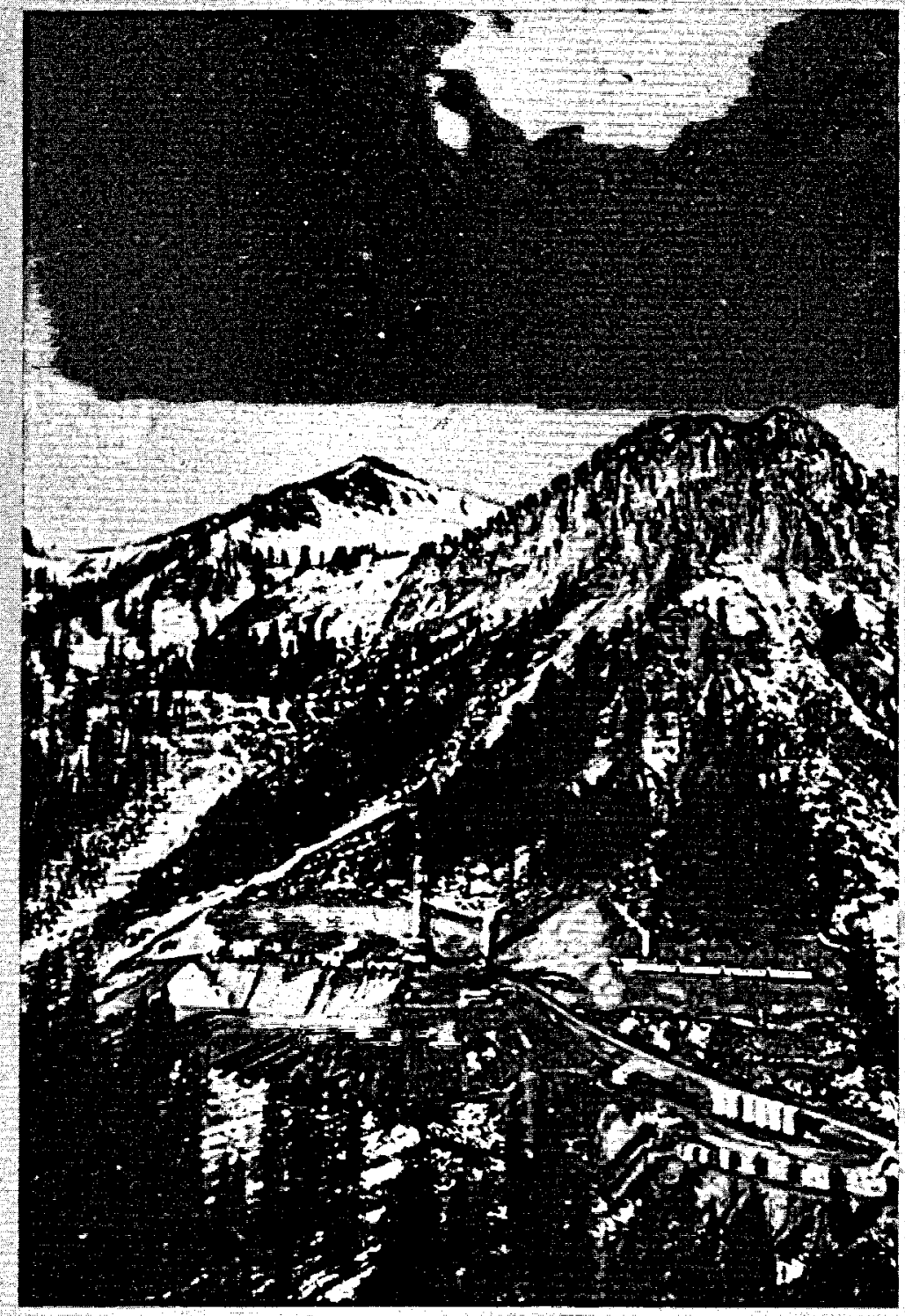

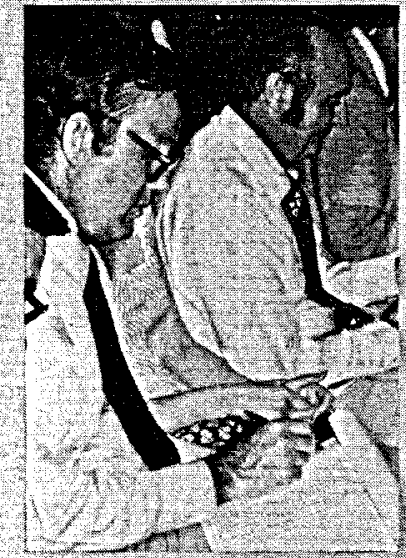

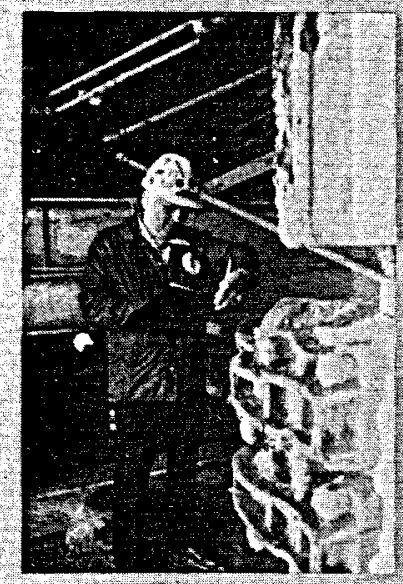

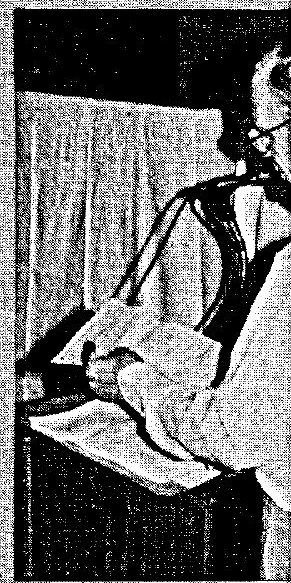

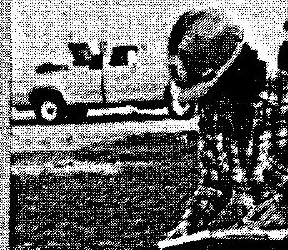




\section{MINERAL REVENUE MANAGEMENT IN 1983}

The year was one of dramatic change for the Federal Government's management of revenues from Federal and Indian lands. Many of the provisions of the Federal Oil and Gas Royalty Management Act of 1982 (FOGRMA) took effect during 1983, including the provisions for monthly payments of royal ties to those $S$ tates that share Federal onshore revenues. Additionally, the Act requires that detailed information by lease be made available to each $\mathrm{S}$ tate and Indian Tribe that receives royal ty revenues.

During 1983 the Royalty Management Program's efforts to audit royalty payors continued: $\$ 28.2$ million in royalty revenue was recovered as a result of these audits, with the continuing cooperative effort with the States providing $\$ 9.8$ million of the total. This brings the cumula tive collection of underpaid and unpaid royalties to $\$ 86.1$ million in a little more than 2 years.

In February the Secretary of the Interior dedicated the new fiscal accounting system for royalties, the Auditing and Financial System (AFS). By yearend this system was processing over $\$ 3$ billion in annual royalty revenues. Also at yearend, its companion system for tracking production from Federal and Indian leases, the Production Accounting and Auditing System (PAAS), was being tested through a coopera tive effort with 14 companies who volunteered to report da ta in the new PAAS report format. Thus Royalty Management was able to start trying out the new system on real rather than test data.

Development of a third system, the Bonus and Rental ACcounting Support System (BRASS), was also in process at the end of 1983. BRASS accommodates the Secretarial decision to shift the collection of onshore rentals on nonproducing leases from the Bureau of Land Management to the Minerals Management Service.

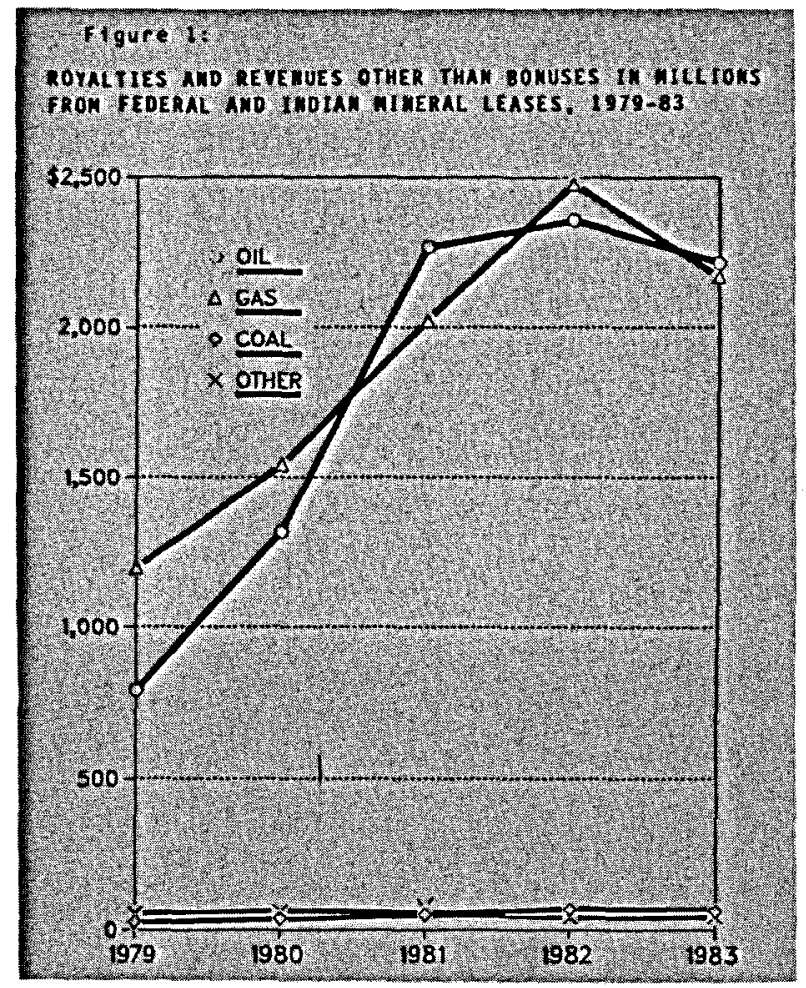


Hence, at the close of 1983, the Department had well underway all its major efforts for overhauling the Federal royalty management process.

In this third annual statistical report we update summary tabulations of all royalty collections from Federal mineral leases in the offshore lands (Table 5) and in the onshore lands (Table 11), as well as those from the Indian mineral leases in the Indian lands ( $\mathrm{Ta}$ ble 14). We also break out from the Federal revenues a detailed account of the royalty-in-kind program (Table 18).

Outer Continental Shelf (OCS) lease bonuses received during 1983 amounted to $\$ 5.749$ billion. Again, this reflects industry's intense interest in offshore oil and gas development and in the Department's expanded offshore leasing program (Table 9). The lease offering in the Central OCS area of the Gulf of Mexico brought in $\$ 3.367$ billion alone-nearly as much as for all OCS areas in 1982.

Onshore leasing declined somewhat from 1982 levels. But the 601 competitive leases that were issued generated over $\$ 62$ million in bonus bids. Of these, 505 were oil and gas leases, 85 geothermal, and 11 coal (Table 12). Another 12,349 leases were issued noncompetitively. All told, the Department of the Interior had more than 152,000 mineral leases, licenses, and permits under supervi-

Table 1-a:

1983 MIIERAL REVEMUES FROM TEDERAL ATD IMDIAN IINERAL LEASES TN THE UNTTED STATES

OFFSHORE IEDERAL LAMDS:

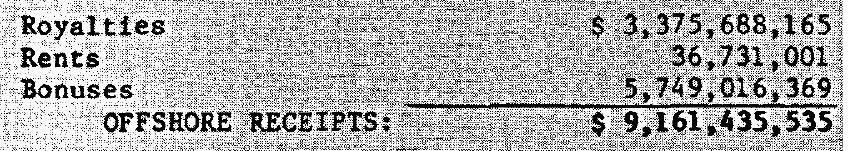

ONSHORE FEDERAT ILWDS :

*Fiscal year data frop Public Land statiscics, 1983 and other Bureau of land Managenent records.
Royalt tes

Rents

Bonuses

ONSHORE RECEIPTS:

INDLN IRIBAL LANDS \& AIIOTHENTS:

Royalties

Rents

Bonuses

INDIAN RECEIPTS:

TOTAL RECETPTS:

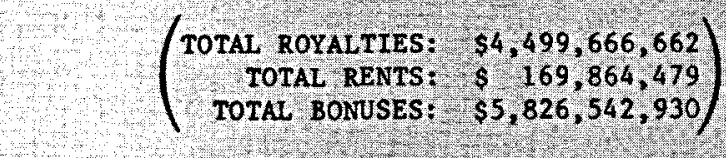

s. $967,851,047$

$129,511,928$

$62,108,205$.

3 1,159,471,180

S. 156,127,450

$3,621,550$

$15,418,356:$

\$. 175,167,356

$\$ 10,496,074,071$ 
Table $1-b$ :

1983 DISBURSEARTS OF INIERAL REYENUES GROA FEDERAL AMD TMDTAY ITERAL LBASES IA TEB DNT TED STATES

OHFSHORE TEDBRAL RECEIPTS:

Histortc Preservation Fund

Land 6 Water Conservation Fund

U.S. Treasury

Wind fall Profit Tax suB TOLAL,
$\$ 150,000,000 *$ $814,693,040 *$

$7,763,568,895$

$433,173,600$

$\$ 9,161,435,535$

ONSHORE TBDERAL RECEIPTS:

Reclamation Fund

State Shares (26. States 1 . 1983)

U.5. Treasury

Wind fall Proflt Tax sUBTOTAL:

S. $350,978,246 * *$

$454,359,341 * t$

$222,689,893$

$131,443,700$

s, $1,159,471,180$

s. $175,167,356$

To Tribes of Allottees SUB TOTAL

$175,167,356$

TOTAL: $\$ 10,496,074,071$

* Fiscal year data reflect prescribed transfers by the Minerals Management Service to U.S. Treasury accounts malntained for these funds.

Fiscal year data frou Public Land statistics, 1983, Bureau of $L$ and Management, and a variety of other sources.

HOTE: AII revenues from wineral leases on Indlan tribal lands (1tst, page 39) or allotuents 80 directly to the respective owners. shares. sion in the onshore Federal and Indian lands as of December 31, 1983 (Table 23).

State revenues from onshore Federal leases decreased 25 percent, from $\$ 609.7$ million in calendar 1982 to $\$ 454.4$ million in calendar 1983 ( $T$ able 16). Indian tribal and allottee royalties decreased from $\$ 162.7$ million to $\$ 156.1 \mathrm{mil}-$ lion during that same period (Table 14, page 46). Nevertheless, mineral leasing revenues continued to be an important source of revenue to Sta te and Indian governments.

Oil and natural gas were the most significant commodities extracted from Federal and Indian lands, as in all previous years. DOI managed 20,717 producible oil and gas leases, which yielded 511 million barrels of crude oil and 5.0 trillion cubic feet of natural gas-together worth more than $\$ 28.6$ billion (Tables $2 \& 19$ ). Production of these two commodities represented 30.6 percent of the natural gas and 16.2 percent of the crude oil produced in the United States during the year (Table 25).

1983 was a depressed year for the minerals industry with better than 22 percent of the total U.S. exploration and production capacity dormant because of sluggish demand and continuing world-wide surpluses. However, continuing interest in both onshore and of fshore leasing gives evidence of industry's commitment to development of the Nation's own energy resources. 
Table 2:

SUMYART Of ROTALTY REVETUES COLLECTED PROH FEDERAL ARD IRDLAM RIMERAL LEASES IN

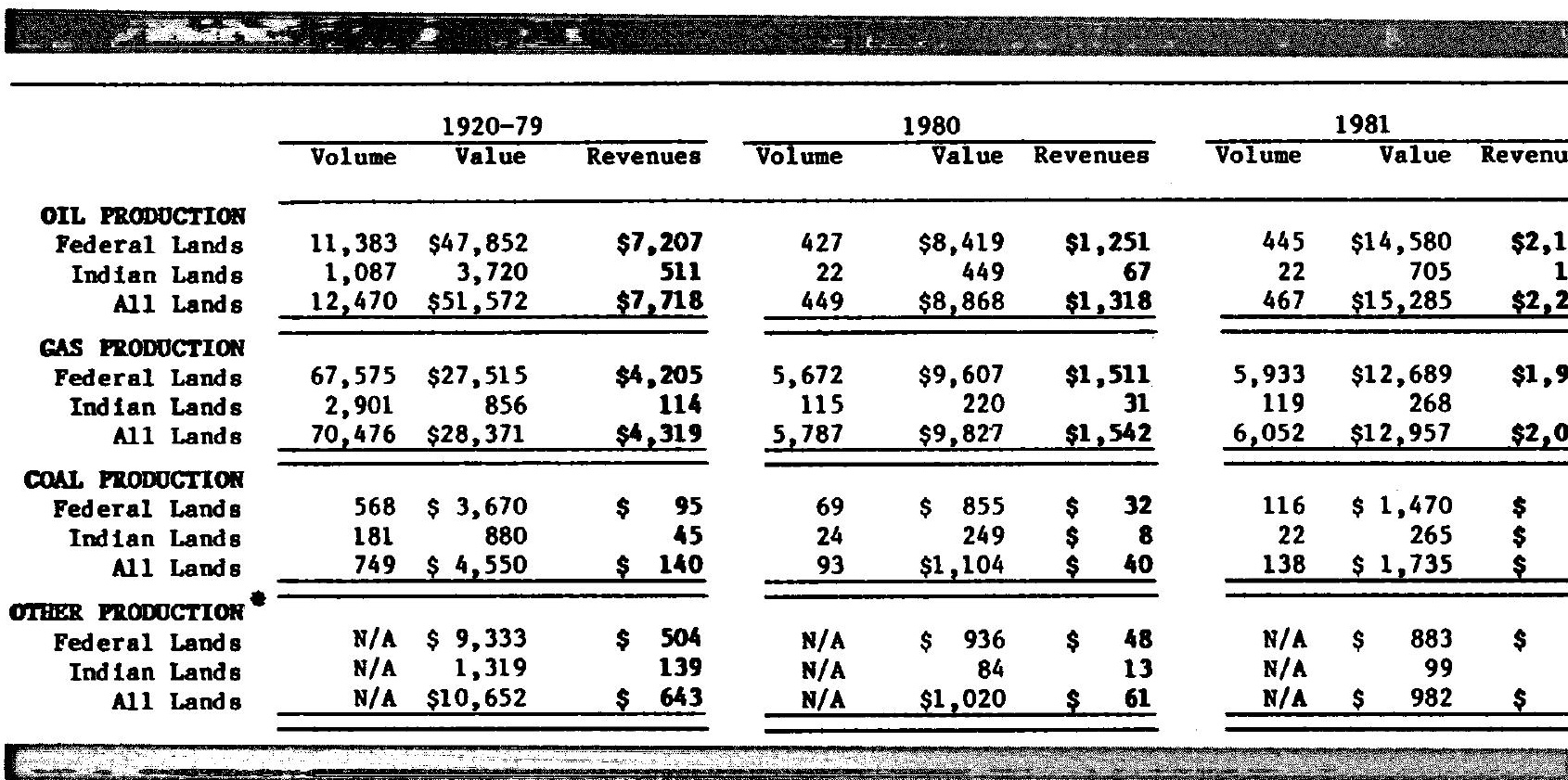

- Ortirg prooucrion includes the following solid and fluld minerals: barite, bentonite, carbon dloxide, chat, clay, copper, feldspar, fluorspar, gas lost, gasoline \& LPG, geothermal energy, gllsonite, gypsum, Iron ore, lead, limestone, molybdenum, oll lost, oll shale, phosphate, potash, quartz crystals, salt, sand-gravel, silfca sand, sodlum, sulfur, uranium, wavellite, and zinc. Certain of these minerals are summarized in Tables 3 and 4.

HOTB: Data are rounded: dollars in millions for both market value and revenues, o11 in millions of barrels (42 U.S. gallons), gas in millions of Mcf (thousand cubic feet), coal in millions of short tons $(\$ 2,000)$.

sourcs: U.S. Geological Survey and MInerals Management Service, Department of the Interior. 
THE UNITED STATES, 1920-83

\begin{tabular}{|c|c|c|c|c|c|c|c|c|}
\hline \multicolumn{3}{|c|}{1982} & \multicolumn{3}{|c|}{1983} & \multicolumn{2}{|l|}{$1920-83$} & \\
\hline olume & Value & Revenues & Volume & Value & Revenues & Volume & Revenues & \\
\hline $\begin{array}{r}471 \\
20 \\
491 \\
\end{array}$ & $\begin{array}{r}\$ 14,425 \\
612 \\
\$ 15,037 \\
\end{array}$ & $\begin{array}{r}\$ 2,265 \\
92 \\
\$ 2,357 \\
\end{array}$ & $\begin{array}{r}491 \\
20 \\
511 \\
\end{array}$ & $\begin{array}{r}\$ 14,142 \\
598 \\
\$ 14,740 \\
\end{array}$ & $\begin{array}{r}\$ 2,127 \\
90 \\
\$ 2,217 \\
\end{array}$ & $\begin{array}{rr}13,217 & \$ 99,418 \\
1,171 & 6,084 \\
14,388 & \$ 105,502 \\
\end{array}$ & $\begin{array}{r}\$ 15,030 \\
864 \\
\$ 15,894 \\
\end{array}$ & $\begin{array}{r}\text { Federal Land } 8 \\
\text { Ind1an Lands } \\
\text { A11 Land } 8\end{array}$ \\
\hline $\begin{array}{r}5,841 \\
133 \\
5,974 \\
\end{array}$ & $\begin{array}{r}\$ 15,201 \\
375 \\
\$ 15,576 \\
\end{array}$ & $\begin{array}{r}\$ 2,424 \\
54 \\
\$ 2,478 \\
\end{array}$ & $\begin{array}{r}4,926 \\
118 \\
5,044 \\
\end{array}$ & $\begin{array}{r}\$ 13,787 \\
355 \\
\$ 14,142 \\
\end{array}$ & $\begin{array}{r}\$ 2,120 \\
51 \\
\$ 2,171 \\
\end{array}$ & $\begin{array}{r}89,947 \$ 78,799 \\
3,386 \quad 2,074 \\
93,333 \$ 80,873 \\
\end{array}$ & $\begin{array}{r}\$ 12,247 \\
287 \\
\$ 12,534 \\
\end{array}$ & $\begin{array}{r}\text { CAS PRODOCTIOd } \\
\text { Federal Land } 8 \\
\text { Ind Ian Land } 8 \\
\text { A11 Land } 8\end{array}$ \\
\hline $\begin{array}{r}101 \\
29 \\
130 \\
\end{array}$ & $\begin{array}{r}\$ 1,521 \\
409 \\
\$ 1,930 \\
\end{array}$ & $\begin{array}{r}\$ \quad 61 \\
\$ \\
\$ \quad 70 \\
\end{array}$ & $\begin{array}{r}105 \\
29 \\
134 \\
\end{array}$ & $\begin{array}{r}\$ 1,550 \\
478 \\
\$ 2,028 \\
\end{array}$ & $\begin{array}{r}56 \\
9 \\
65 \\
\end{array}$ & $\begin{array}{r}959 \$ \quad 9,066 \\
285 \\
1,244 \$ 11,281 \\
\end{array}$ & $\begin{array}{r}\$ 287 \\
80 \\
\$ \quad 367 \\
\end{array}$ & $\begin{array}{r}\text { Federal Land } 8 \\
\text { Indian Land } 8 \\
A 11 \text { Land } 8\end{array}$ \\
\hline $\begin{array}{l}N / A \\
N / A \\
N / A\end{array}$ & $\begin{array}{r}\$ 888 \\
60 \\
\$ \quad 948 \\
\end{array}$ & $\begin{array}{r}37 \\
7 \\
44 \\
\end{array}$ & $\begin{array}{l}N / A \\
N / A \\
N / A\end{array}$ & $\begin{array}{r}739 \\
79 \\
\$ \quad 818 \\
\end{array}$ & $\begin{array}{r}40 \\
6 \\
46 \\
\end{array}$ & $\begin{array}{rr}\text { N/A } \$ 12,779 \\
\text { N/A } 1,641 \\
\text { N/A } \$ 14,420 \\
\end{array}$ & $\begin{array}{r}\$ \quad 672 \\
177 \\
\$ \quad 849 \\
\end{array}$ & $\begin{array}{r}\text { Federal Lands } \\
\text { Indian Lands } \\
\text { All Lands }\end{array}$ \\
\hline
\end{tabular}

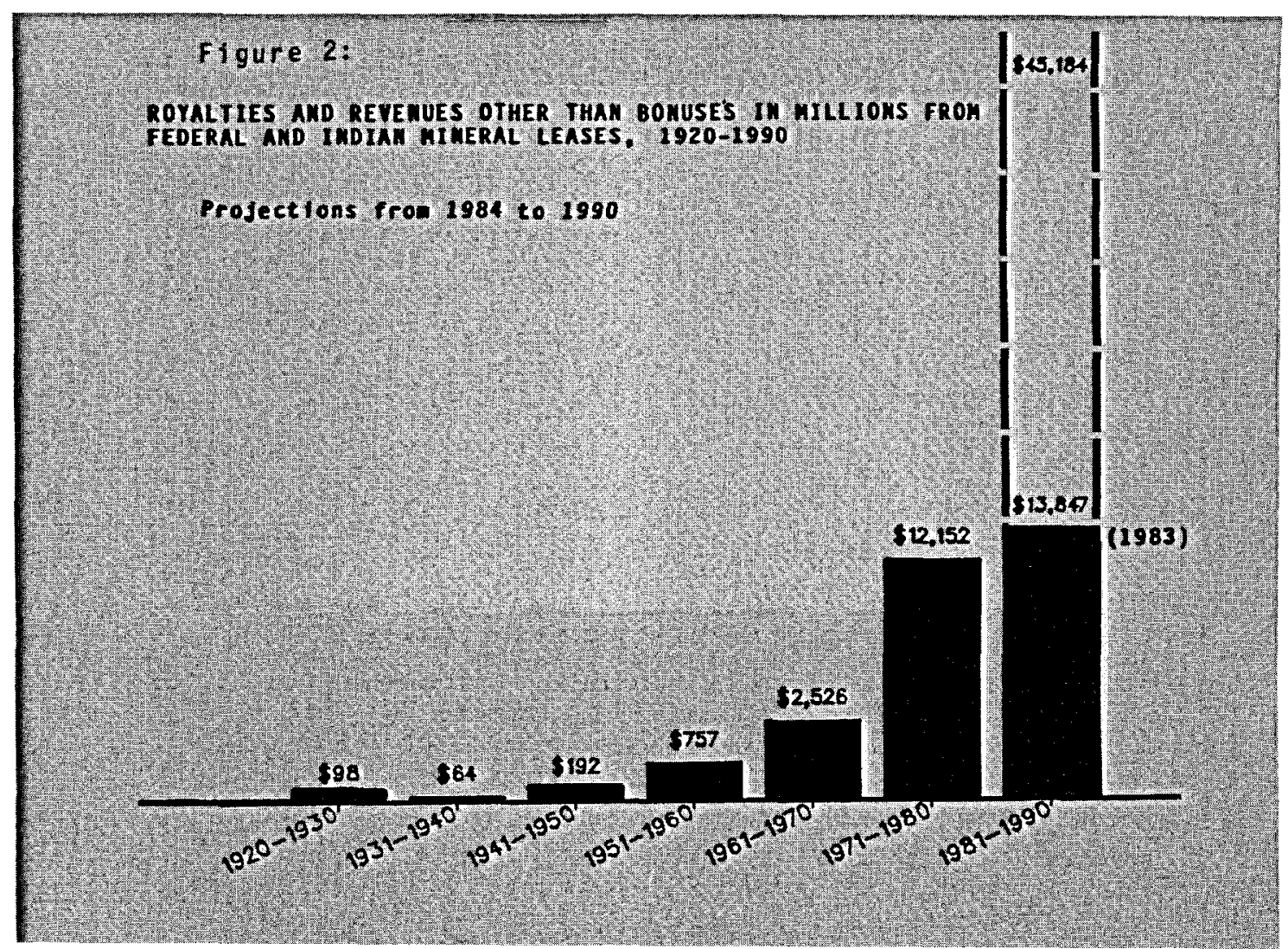




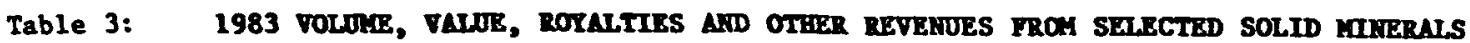
OTHER THAR COAL FROA FEDERAT ATD IDDTAR LBASES

\begin{tabular}{|c|c|c|c|}
\hline & $\begin{array}{l}\text { VOLUME } \\
\text { IN TONS }\end{array}$ & $\begin{array}{l}\text { MARKET } \\
\text { VALUE }\end{array}$ & $\begin{array}{l}\text { REVENUES } \\
\text { RECEIVED }\end{array}$ \\
\hline \multirow[t]{2}{*}{$\begin{array}{l}\text { Sodiun } \\
\text { Potash } \\
\text { Lead } \\
\text { Uraniua } \\
\text { Zinc } \\
\text { Phosphate } \\
\text { Copper }\end{array}$} & $\begin{array}{c}4,209,628 \\
3,094,286 \\
493,943 \\
460,866 \star \\
145,362 \\
1,802,969 \\
11,135 \\
\end{array}$ & $\begin{array}{r}\$ 207,935,306 \\
195,411,233 \\
59,101,000 \\
59,000,813 \\
31,263,384 \\
21,677,590 \\
2,099,703 \\
\end{array}$ & $\begin{array}{r}\$ 9,850,697 \\
6,607,154 \\
2,951,458 \\
8,612,862 \\
1,560,541 \\
1,086,961 \\
104,985\end{array}$ \\
\hline & $\mathbf{T}$ & $\$ 576,489,029$ & $\$ 30,774,658$ \\
\hline
\end{tabular}

*Weight of unprocessed ore at primary point of delivery.

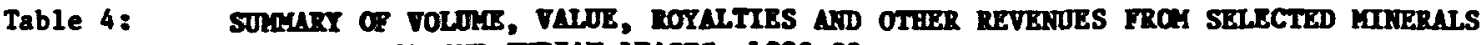

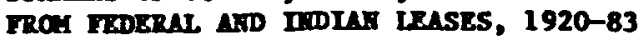

\begin{tabular}{lcc}
\hline PRODUCTION & MARKET & REVENUES \\
VOLUME & VALUE & RECEIVED \\
\hline
\end{tabular}

\begin{tabular}{|c|c|c|c|c|}
\hline $\begin{array}{l}\text { FLUID MINERALS: } \\
\text { Gasoline C LPG } \\
\text { Sulfur } \\
\text { Salt } \\
\text { Carbon Dioxide } \\
\text { O11 Lost } \\
\text { Ges Lost } \\
\text { Geotheral* } \\
\text { Hot Water* }\end{array}$ & $\begin{array}{r}33,258,261,498 \\
18,626,616 \\
4,765,679 \\
11,834,353 \\
213,203 \\
162,608,340 \\
2,454,667,260 \\
26,638,200\end{array}$ & $\begin{array}{l}\text { gal. } \\
\text { t. } \\
\text { t. } \\
\text { Mcf. } \\
\text { bbl. } \\
\text { Mcf. } \\
\text { kWh. } \\
\text { gal. }\end{array}$ & $\begin{array}{r}\$ 3,473,436,017 \\
411,806,509 \\
1,494,712 \\
2,352,606 \\
2,231,170 \\
112,140,965 \\
56,275,862 \\
409,896\end{array}$ & $\begin{array}{r}\$ 217,458,897 \\
46,034,990 \\
235,150 \\
242,523 \\
272,482 \\
16,338,305 \\
7,032,416 \\
40,991\end{array}$ \\
\hline \multirow[t]{2}{*}{$\begin{array}{l}\quad \text { SOLID MINERALS: } \\
\text { Copper } \\
\text { Fluorspar } \\
\text { Lead } \\
\text { Linestone } \\
\text { Phosphate } \\
\text { Potash } \\
\text { Sand-Gravel } \\
\text { Sodium } \\
\text { Uranium } \\
\text { Zinc } \\
\text { Hiscellany }\end{array}$} & $\begin{array}{r}381,508 \\
322,423 \\
11,458,012 \\
3,620,612 \\
116,582,593 \\
131,669,551 \\
144,809,335 \\
70,117,614 \\
34,334,199 \\
1,397,114 \\
20,345,148\end{array}$ & $\begin{array}{l}t . \\
t . \\
t . \\
t . \\
t . \\
t . \\
t . \\
t . \\
t . \\
t .\end{array}$ & $\begin{array}{r}181,999,546 \\
20,678,370 \\
1,789,608,625 \\
5,709,780 \\
587,470,463 \\
3,769,318,582 \\
154,681,969 \\
2,792,920,809 \\
737,533,508 \\
271,522,287 \\
90,488,411\end{array}$ & $\begin{array}{r}13,438,988 \\
832,145 \\
92,165,212 \\
296,921 \\
38,493,863 \\
151,845,210 \\
12,989,770 \\
127,160,901 \\
104,164,549 \\
12,560,744 \\
5,797,155\end{array}$ \\
\hline & $\mathbf{T} 0 \mathbf{T}$ & & $\$ 14,462,080,087$ & $\$ 847,401,212$ \\
\hline
\end{tabular}

*Energy extracted from geothermal or heated water sources is measured in terms of Btu's, but is accounted for commerclally in kllowatt hours or gallons.

NOT: "Miscellany" also Includes certain other minerals footnoted in Table 2. 


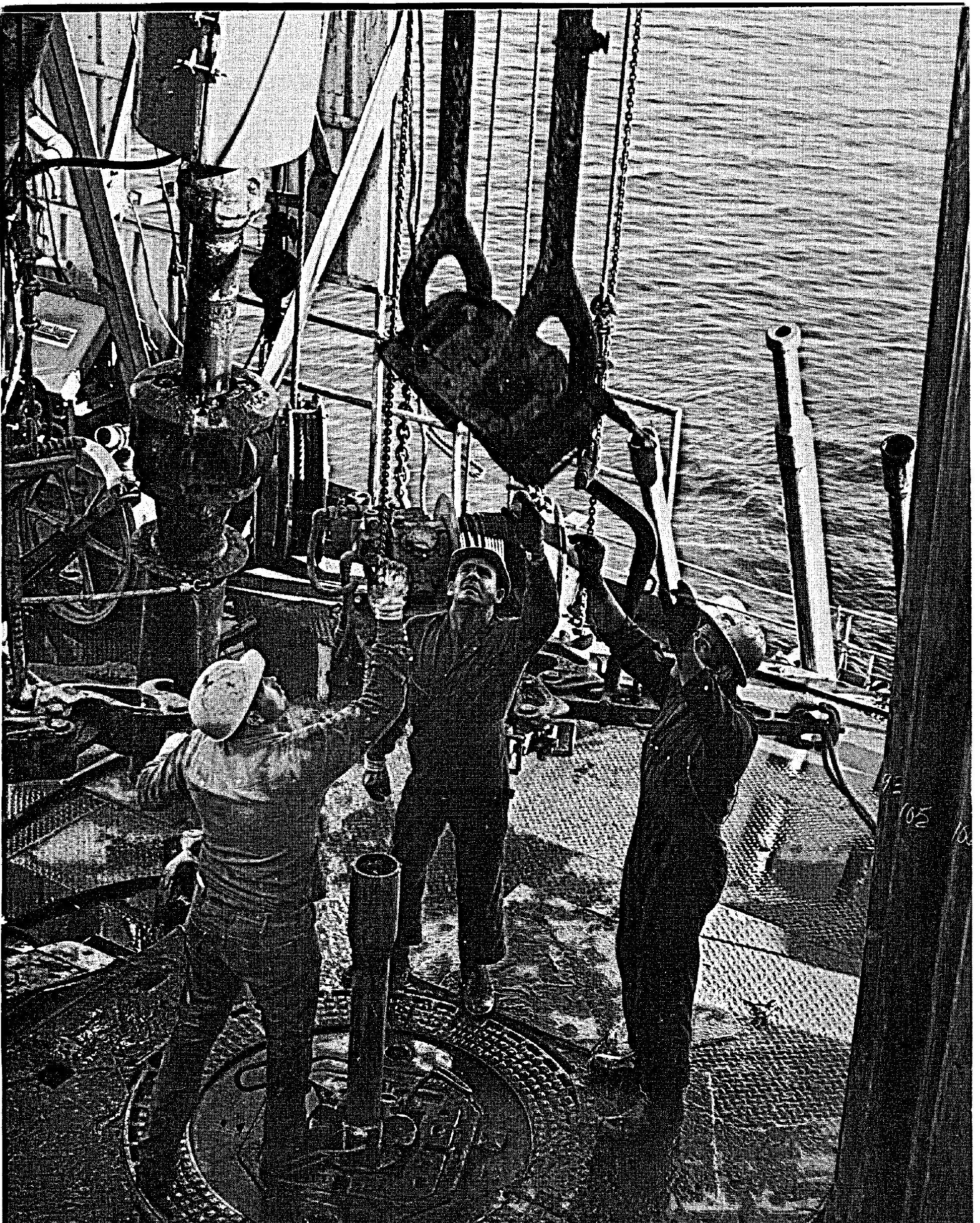




\section{OFFSHORE FEDERAL MINERAL REVENUES}

Offshore oil and gas bonuses and royalties provided the majority of Federal mineral leasing revenues again in 1983. The Department held 7 new lease sales and the second phase of the last 1982 sale, resulting altogether in $\$ 5.749$ billion in lease bonuses paid and 1,251 new leases issued. At the end of the year, there were 3,787 active oil and gas leases on the Outer Continental Shelf (Table 22) and more than one-third were in production. These 1,381 leases yielded 341 million barrels of oil (up 6.2 percent from 1982), 3.9 trillion cubic feet of natural gas (down 15.8 percent from the previous year), and $\$ 3.4$ billion in rental and royalty income. Gas output remained depressed as production continued to fall throughout the year. In 1983, 10 percent of the oil and 24 percent of the gas produced in the United States came from Federal OCS leases.

Revenues from Federal offshore oil and gas production now represent 72 percent of all oil royalties and 82 percent of all gas royalties. These revenues are expected to increase as the Department's leasing initiatives accelerate development of new fields over the next decade.

As the accelerated 5-year program completed its second year, expanded leasing continued to show results on the California OCS, al though at a slower pace. Oil production increased from 28 to 30 million barrels during the past year. Market value increased proportionately, from $\$ 679$ million to $\$ 709$ million. Oil royalties were up slightly--1.3 percent. For gas there was a substantial increase in royalties--up 56 percent, to $\$ 17.1$ from

Flgure 3:

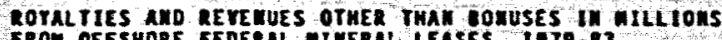
FROA OF FHORE FEOERAL NIWERAL LEASES, I579-83

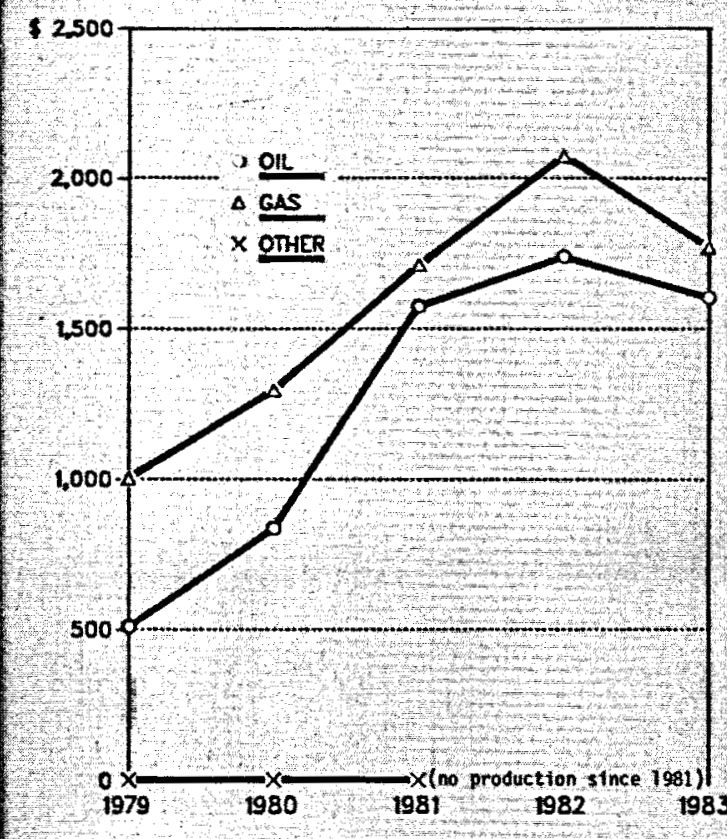

$\$ 10.9$ million for the area. Gas production increased also--from 17.7 to 23.2 million Mcf. Moreover, market value climbed from $\$ 41$ to $\$ 66$ million because of gas price increases. Revenues were up 4.9 percent overall-from $\$ 171.9$ to $\$ 180.2$ million for oil and gas (Table 7).

In spite of lower demands for both oil and gas, 1983 was a good year for offshore oil and gas because it was a year highlighted by the first effects of the Department's new areawide program for the OCS. Additional discoveries in the offshore California waters further enhanced that area's potential 
as a major source of oil and gas reserves through the end of the century.

The Central Gulf of Mexico sale on May 25 sparked industry's largest response ever to a single offshore sale since passage of the OCS Lands Act of 1953. The 7,050 tract offering equalled the entire number offered during the
22-1/2 years between October 13, 1954, and April 13, 1976. The May sale brought in $\$ 3,367,606,134$ in bonus monies alone. Altogether, the 1983 offerings in the Alaska, Atlantic, Gulf, and Pacific OCS resulted in $\$ 5.7$ billion in bonuses for the U.S. Treasury and 6.6 million acres leased to the minerals industry (Tables 9 \& 10).

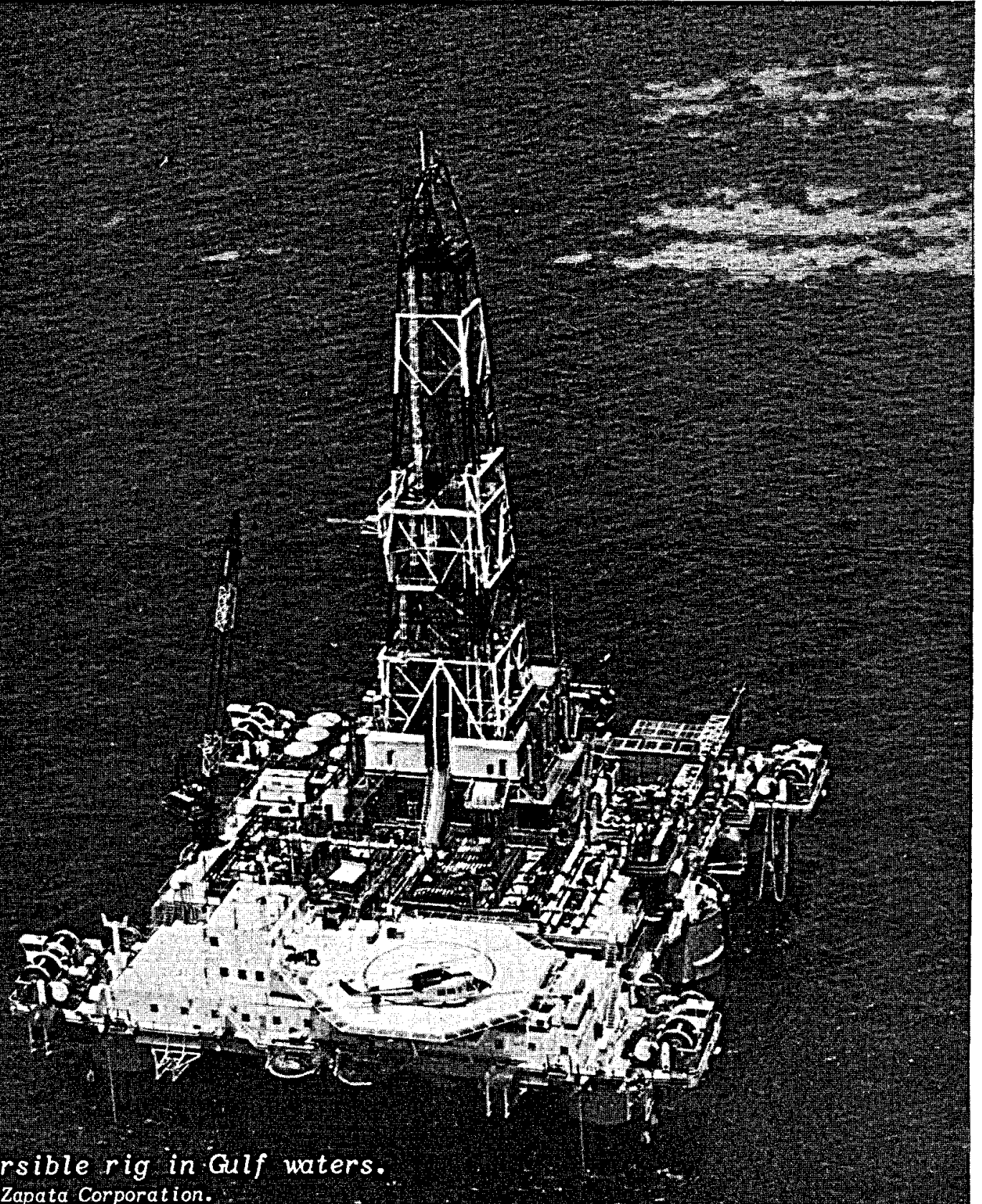

Semi subrersible rig in Gulf waters. 
10 : OFFSHORE MINERAL REVENUES

Table 5:

1983 VOLJRE, VALUE, ROTALTIES AKD OTHER REVENUES FROM OFFSHORE FEDERAL MINERAL LEASES BY OCS AREA ARD COMOMODTT

OIL IN BARRELS/GAS IN MCF

REVENUES

Volume Market Value

RECEIVED

OFPSHORE CALIFORHLA

011

$30,169,217$

\$ $708,976,600$

$23,159,696$

$65,773,537$

Other

N/A

$\$$

\$ $163,064,618$

$17,101,120$

$\$$

o

S U B T O T A L :

$\$$

$774,750,137$

$\$ 180,165,738$

OFFSEORE LOUISLAKA

011

Gas

Other
$\$ 8,738,847,500$ $8,397,629,750$

s
$\$ 1,349,359,400$

$1,343,619,320$

\$

$\$ 2,692,978,720$

OFFSHORE TEXAS

011

Gas

Other
$\$ 17,136,477,250$

S U B T O TA L :

\begin{tabular}{rrr}
$19,723,885$ & $\$$ & $592,899,900$ \\
$829,306,204$ & & $2,529,383,920$ \\
N/A & $\$$ & 0 \\
\hline
\end{tabular}

S U B T O TA L :

$\$ 3,122,283,820$

$\$ 21,033,511,207$

TOT A L :
\$ $\quad 91,518,820$

$411,024,887$

\$

$\$ \quad 502,543,707$

$\$ 3,375,688,165$

1983 SUMPARY OF VOLUME, VALUE, ROTALTIES AND OTHER REVERUES FROM OFFSHORE FEDERAL MTIEERAL LEASES BY COMAODITY

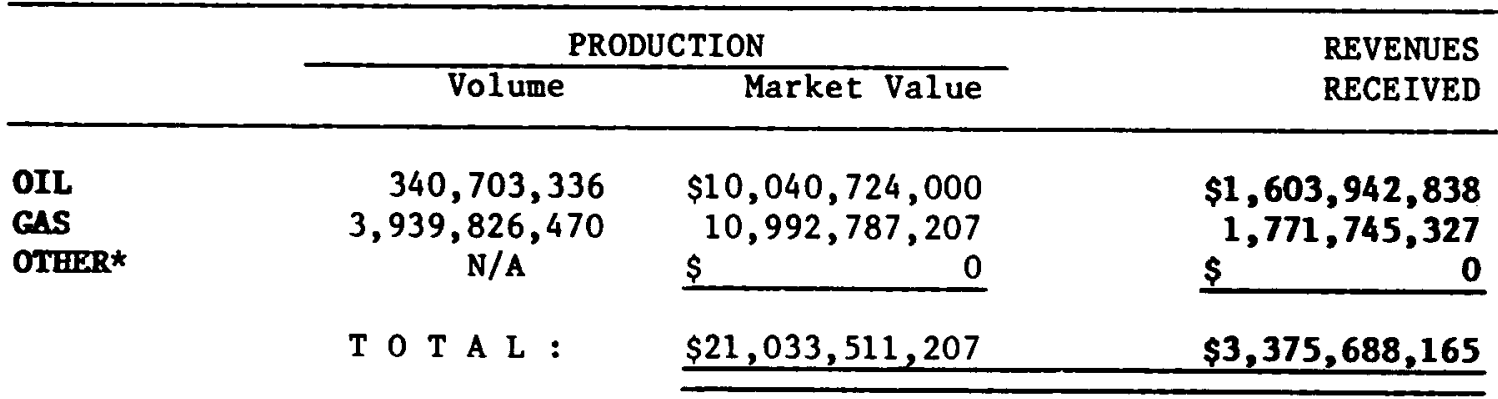

* No production in 1983.

NOTE: "Other" includes certain of the fluid minerals footnoted in Table 2. 
Table 6:

SUMAARY OF TOTAL VOLUKE, VALUE, ROYALTIES AND OTHER REVEHUES FROM OFFSHORE FEDERAL MTHERAL LEASES BY OCS AREA AND COMYODITY, 1953-83

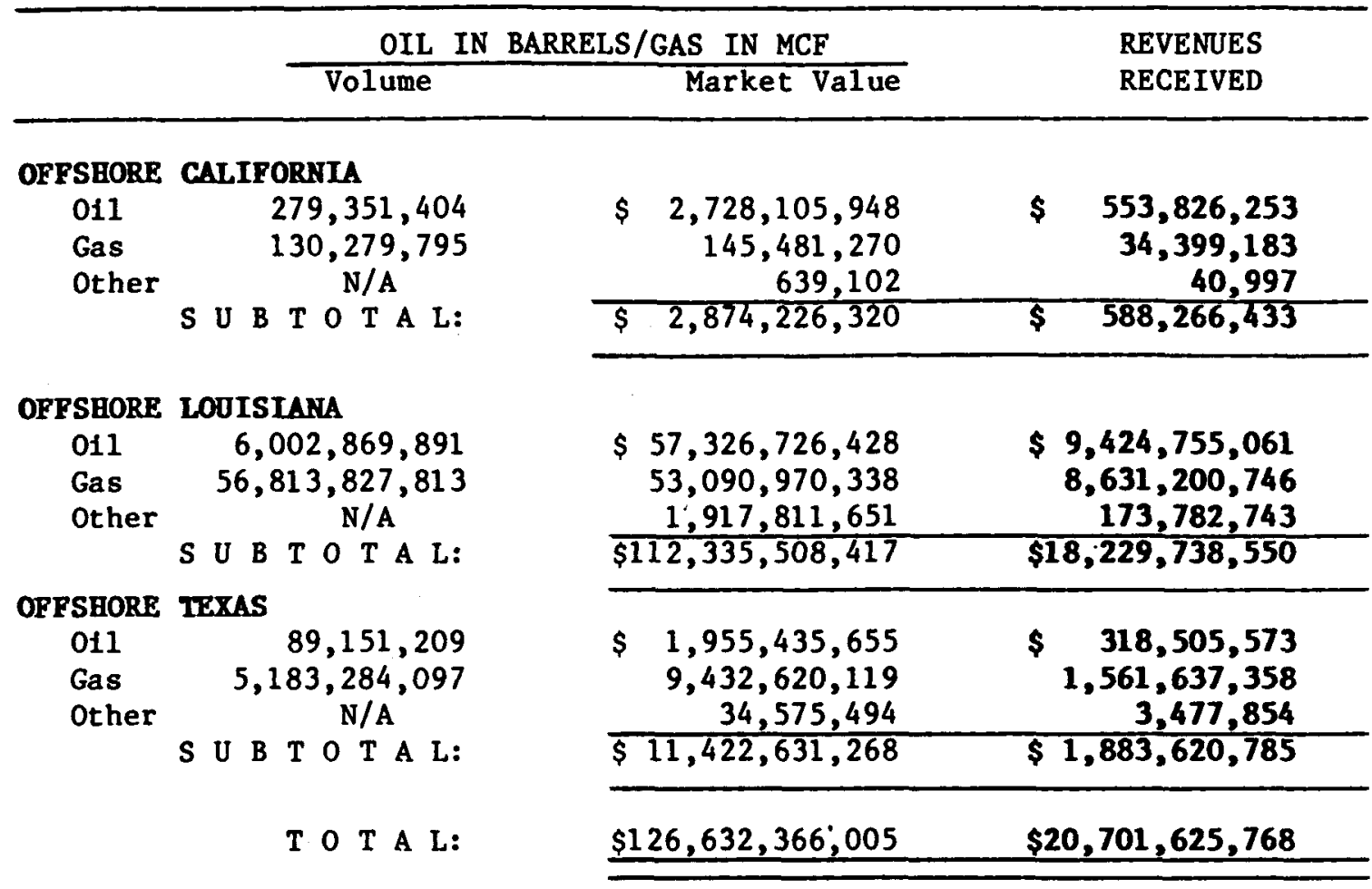

SUMMARY OF TOTAL VOLUME, VALUE, ROYALTIES AND OTHER REVENUES FROY OFFSHORE FEDERAL MINERAL LEASES BY COMMODITY, 1953-83

\begin{tabular}{|c|c|c|c|}
\hline & \multicolumn{2}{|c|}{ PRODUCTION } & \multirow{2}{*}{$\begin{array}{l}\text { REVENUES } \\
\text { RECEIVED }\end{array}$} \\
\hline & Volume & Market Value & \\
\hline \multirow[t]{2}{*}{$\begin{array}{l}\text { OIL } \\
\text { GAS } \\
\text { OTHER }\end{array}$} & $\begin{array}{c}6,371,372,504 \\
62,127,391,705 \\
\text { N/A }\end{array}$ & $\begin{array}{r}\$ 2,010,268,031 \\
62,669,071,727 \\
1,953,026,247 \\
\end{array}$ & $\begin{array}{r}\$ 10,297,086,887 \\
10,227,237,287 \\
177,301,594 \\
\end{array}$ \\
\hline & T O T A L: & $\$ 126,632,366,005$ & $\$ 20,701,625,768$ \\
\hline
\end{tabular}

NOTE: "Other" Includes certain of the fluid minerals footnoted in Table 2 . 
Table 7:

SUMRARY OF VOLDKE, VALUE, ROTALTIES AND OTHER REVENUES FROM OFFSHORE FEDERAL MTINERAL LEASES BT OCS AREA AID TEAR, 1953-83

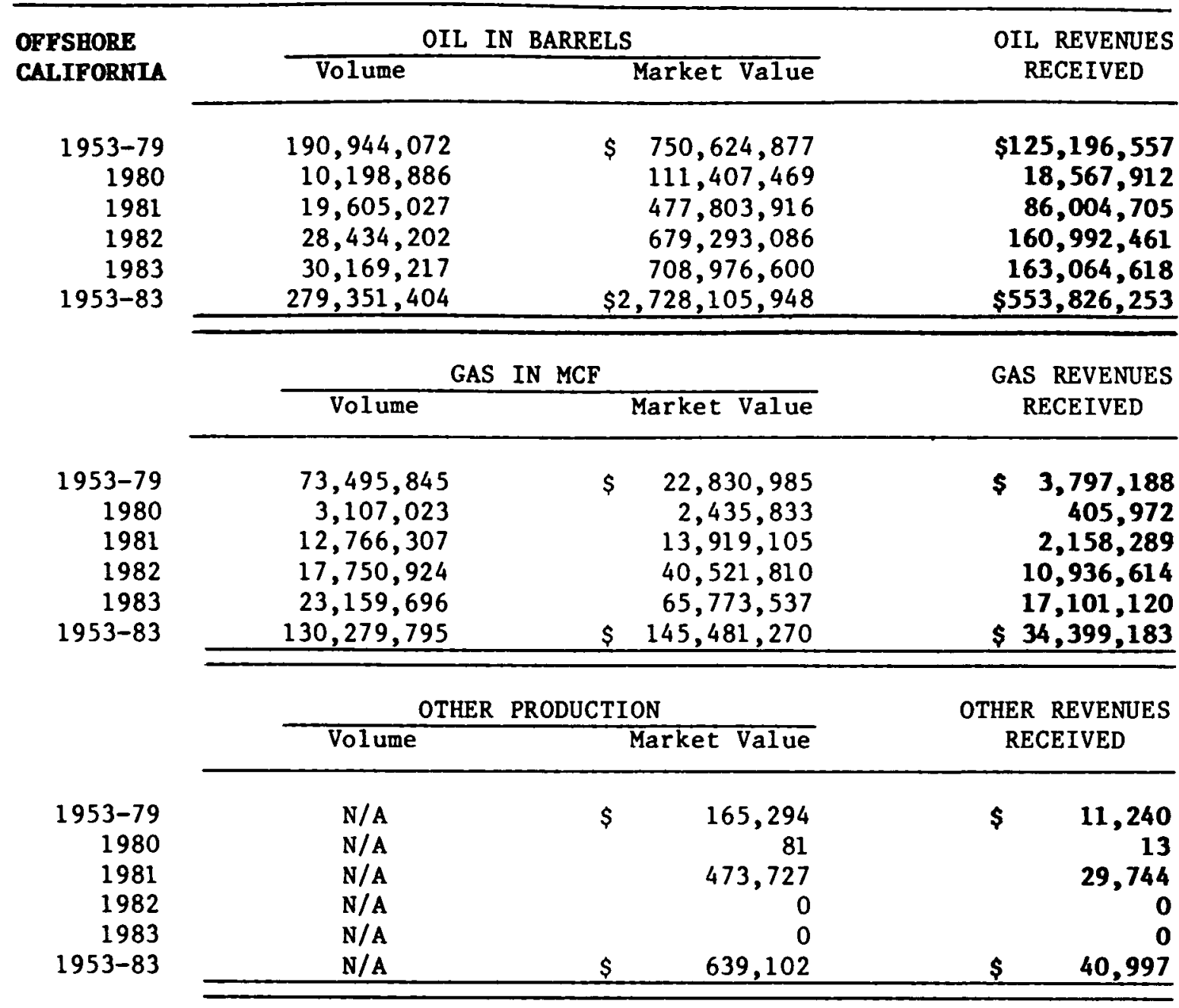

HOTE: "Other" Includes certain of the fluid minerals footnoted in Table 2 . 
Table 7 (cont'd):

SUMAARY OF VOLUYE, VALUE, ROYALTIES AND OTHER REVENUES FRCA OFFSHORE FEDERAL MTIERAL LEASES BY OCS AREA AND YEAR, 1953-83

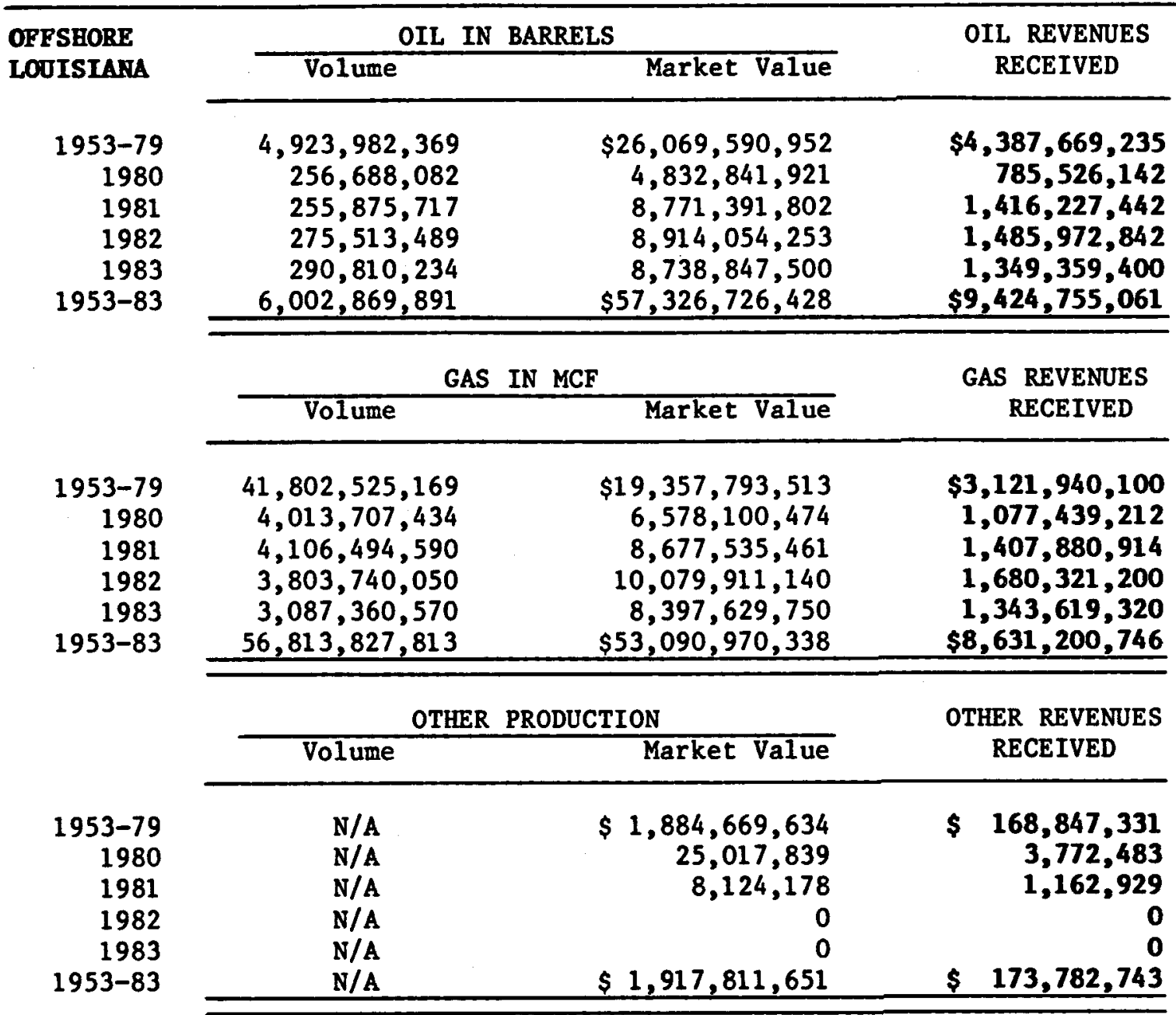


Table 7 (cont'd):

SULAARY OF VOLUIE, VALUE, ROTALTIES ATD OTHER REVENUES FROA OPFSHORE FEDERAL MTIERAL LEASES BY OCS AREA AND YEAR, 1953-83

\begin{tabular}{|c|c|c|c|}
\hline \multirow{3}{*}{$\begin{array}{l}\text { OFFSHORE } \\
\text { TEXAS } \\
\\
1953-79 \\
1980 \\
1981 \\
1982 \\
1983 \\
1953-83\end{array}$} & \multicolumn{2}{|c|}{ OIL IN BARRELS } & \multirow{2}{*}{$\begin{array}{l}\text { OIL REVENUES } \\
\text { RECEIVED }\end{array}$} \\
\hline & \multirow{2}{*}{$\begin{array}{l}\text { Volume } \\
27,376,890 \\
10,502,007 \\
14,284,661 \\
17,263,766 \\
19,723,885 \\
89,151,209 \\
\end{array}$} & Market Value & \\
\hline & & $\begin{array}{r}165,277,711 \\
198,862,396 \\
438,571,199 \\
559,824,449 \\
592,899,900 \\
\$ 1,955,435,655\end{array}$ & $\begin{array}{r}27,436,324 \\
33,143,332 \\
73,084,362 \\
93,322,735 \\
91,518,820 \\
\$ \quad 318,505,573 \\
\end{array}$ \\
\hline & \multicolumn{2}{|c|}{ GAS IN MCF } & $\begin{array}{l}\text { GAS REVENUES } \\
\text { RECEIVED }\end{array}$ \\
\hline \multirow[t]{2}{*}{$\begin{array}{r}1953-79 \\
1980 \\
1981 \\
1982 \\
1983 \\
1953-83\end{array}$} & $\begin{array}{r}2,141,039,238 \\
624,642,526 \\
730,275,831 \\
858,020,298 \\
829,306,204 \\
5,183,284,097 \\
\end{array}$ & $\begin{array}{r}\$ 1,487,531,140 \\
1,304,676,689 \\
1,811,533,970 \\
2,299,494,400 \\
2,529,383,920 \\
\$ 9,432,620,119 \\
\end{array}$ & $\begin{array}{r}247,916,829 \\
217,446,169 \\
301,923.690 \\
383,325,783 \\
411,024,887 \\
\$ 1,561,637,358 \\
\end{array}$ \\
\hline & $\frac{\text { OTE }}{\text { Volume }}$ & $\frac{\text { TION }}{\text { Market Value }}$ & $\begin{array}{l}\text { OTHER REVENUES } \\
\text { RECEIVED }\end{array}$ \\
\hline $\begin{array}{r}1953-79 \\
1980 \\
1981 \\
1982 \\
1983 \\
1953-83\end{array}$ & $\begin{array}{l}\text { N/A } \\
\text { N/A } \\
\text { N/A } \\
\text { N/A } \\
\text { N/A } \\
\text { N/A }\end{array}$ & $\begin{array}{r}31,932,521 \\
2,173,130 \\
469,843 \\
0 \\
0 \\
34,575,494 \\
\end{array}$ & $\begin{array}{r}3,037,515 \\
362,190 \\
78,149 \\
0 \\
0 \\
3,477,854 \\
\end{array}$ \\
\hline
\end{tabular}

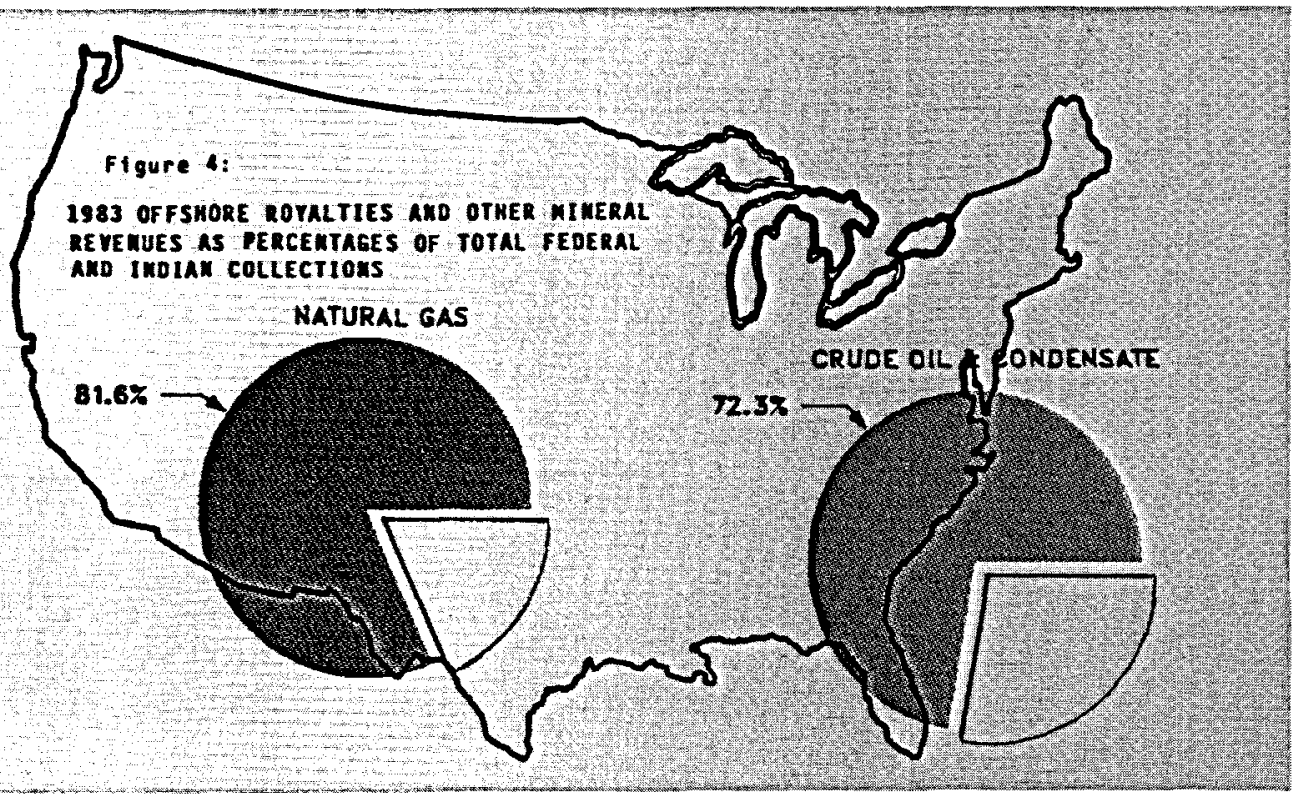


Table 8:

SUMMARY OF ALL POLUNE, VALUE, ROYALTIES AND OTRER REVENUES FROM ALL OFFSHORE FEDERAL MINERAL LEASES, 1953-83

\begin{tabular}{|c|c|c|c|}
\hline $\begin{array}{l}\text { PRODUCTION } \\
\text { VOLUME }\end{array}$ & OIL (bbl) & GAS (Mcf) & OTHER \\
\hline $\begin{array}{r}1953-79 \\
1980 \\
1981 \\
1982 \\
1983 \\
1953-83\end{array}$ & $\begin{array}{r}5,142,303,331 \\
277,388,975 \\
289,765,405 \\
321,211,457 \\
340,703,336 \\
6,371,372,504 \\
\end{array}$ & $\begin{array}{r}44,017,060,252 \\
4,641,456,983 \\
4,849,536,728 \\
4,679,511,272 \\
3,939,826,470 \\
62,127,391,705 \\
\end{array}$ & $\begin{array}{l}\text { N/A } \\
\text { N/A } \\
\text { N/A } \\
\text { N/A } \\
\text { N/A } \\
\text { N/A }\end{array}$ \\
\hline $\begin{array}{l}\text { MARRET } \\
\text { VALUE }\end{array}$ & OIL & GAS & OTHER \\
\hline $\begin{array}{r}1953-79 \\
1980 \\
1981 \\
1982 \\
1983 \\
1953-83\end{array}$ & $\begin{array}{r}\$ 26,985,493,540 \\
5,143,111,786 \\
9,687,766,917 \\
10,153,171,788 \\
10,040,724,000 \\
\$ 62,010,268,031 \\
\end{array}$ & $\begin{array}{r}\$ 20,868,155,637 \\
7,885,212,996 \\
10,502,988,536 \\
12,419,927,350 \\
10,992,787,207 \\
\$ 62,669,071,726 \\
\end{array}$ & $\begin{array}{r}\$ 1,916,767,449 \\
27,191,050 \\
9,067,748 \\
0 * \\
0 * \\
\$ 1,953,026,247 \\
\end{array}$ \\
\hline $\begin{array}{l}\text { REVENUES } \\
\text { RECEIVED }\end{array}$ & OIL & GAS & OTHER \\
\hline $\begin{array}{r}1953-79 \\
1980 \\
1981 \\
1982 \\
1983 \\
1953-83\end{array}$ & $\begin{array}{r}\$ 4,540,302,116 \\
837,237,386 \\
1,575,316,509 \\
1,740,288,038 \\
1,603,942,838 \\
\$ 10,297,086,887\end{array}$ & $\begin{array}{r}\$ 3,373,654,117 \\
1,295,291,353 \\
1,711,962,893 \\
2,074,583,597 \\
1,771,745,327 \\
\$ 10,227,237,287\end{array}$ & $\begin{array}{r}171,896,086 \\
4,134,686 \\
1,270,822 \\
0^{*} \\
0^{*} \\
\$ \quad 177,301,594 \\
\end{array}$ \\
\hline
\end{tabular}

* No production in 1982 or 1983.

HOTE: "Other" includes certain of the fluid minerals footnoted in Table 2 . 
Table 9:

1983 bONUSES PAID OA SAIES OP OFFSHORE FEDERAL OIL \& GAS LRASES BT OCS AREAS

\begin{tabular}{|c|c|c|c|c|c|c|}
\hline & & $\begin{array}{r}\text { Sale } \\
\text { No. }\end{array}$ & $\begin{array}{l}\text { Sale } \\
\text { Date }\end{array}$ & $\begin{array}{l}\text { Tracts } \\
\text { Leased }\end{array}$ & $\begin{array}{l}\text { Acres } \\
\text { Leased } \\
\end{array}$ & $\begin{array}{l}\text { Bonuses } \\
\text { Paid }\end{array}$ \\
\hline \multicolumn{7}{|l|}{ ALASKA: } \\
\hline $\begin{array}{l}\text { Norton Sound } \\
\text { Salnt George }\end{array}$ & Basin & $\begin{array}{l}57 \\
70\end{array}$ & $\begin{array}{l}03 / 15 / 83 \\
04 / 12 / 83\end{array}$ & $\begin{array}{l}59 \\
96\end{array}$ & $\begin{array}{l}335,898 \\
546,546\end{array}$ & $\begin{array}{l}317,873,372 \\
426,458,830\end{array}$ \\
\hline \multicolumn{7}{|l|}{ ATLANTIC: } \\
\hline $\begin{array}{l}\text { Mid-Atlantic } \\
\text { S. Atlantic }\end{array}$ & & $\begin{array}{l}76 \\
78\end{array}$ & $\begin{array}{l}04 / 26 / 83 \\
07 / 26 / 83\end{array}$ & $\begin{array}{l}37 \\
11\end{array}$ & $\begin{array}{r}210,641 \\
62,625\end{array}$ & $\begin{array}{l}68,410,240 \\
13,362,040\end{array}$ \\
\hline \multicolumn{7}{|l|}{ EULF OP MEXICO: } \\
\hline $\begin{array}{l}\text { Area-Wide } \\
\text { Central } \\
\text { West }\end{array}$ & & $\begin{array}{l}69(2) * \\
72 \\
74\end{array}$ & $\begin{array}{l}03 / 08 / 83 \\
05 / 25 / 83 \\
08 / 24 / 83\end{array}$ & $\begin{array}{r}11 \\
623 \\
406\end{array}$ & $\begin{array}{r}58,120 \\
3,089,872 \\
2,246,005\end{array}$ & $\begin{array}{r}37,570,900 \\
3,367,606,134 \\
1,501,712,517\end{array}$ \\
\hline PACIPIC: & & 73 & $12 / 20 / 83$ & 8 & 43,799 & $16,022,336$ \\
\hline & & $\mathbf{T}$ & O T A L : & 1,251 & $6,593,506$ & $\$ 5,749,016,369$ \\
\hline
\end{tabular}

* Sale 69(1), the first phase of this 1982 sale, was held November 17,1982 .

Table 10:

SUMAARY OR BONUSES PAID ON SALES OP OFTSBORE FEDERAL OIL \& GAS LEASES, 1954-83

\begin{tabular}{|c|c|c|c|c|c|c|c|c|c|}
\hline & $\begin{array}{l}\text { No. of } \\
\text { Sales } \\
\end{array}$ & $\begin{array}{l}\text { Tracts } \\
\text { Leased } \\
\end{array}$ & $\begin{array}{l}\text { Acres } \\
\text { Leased } \\
\end{array}$ & $\begin{array}{c}\text { Bonuses } \\
\text { Pald } \\
\end{array}$ & & $\begin{array}{l}\text { No. of } \\
\text { Sales } \\
\end{array}$ & $\begin{array}{l}\text { Tracts } \\
\text { Leased } \\
\end{array}$ & $\begin{array}{l}\text { Acres } \\
\text { Leased } \\
\end{array}$ & $\begin{array}{c}\text { Bonuses } \\
\text { Paid } \\
\end{array}$ \\
\hline $\begin{array}{l}1954 \\
1955 \\
1959 \\
1960 \\
1962 \\
1963 \\
1964 \\
1965 \\
1966 \\
1967 \\
1968 \\
1969 \\
1970\end{array}$ & $\begin{array}{l}3 \\
1 \\
2 \\
2 \\
3 \\
1 \\
2 \\
1 \\
3 \\
2 \\
3 \\
3 \\
2\end{array}$ & $\begin{array}{r}114 \\
121 \\
42 \\
148 \\
420 \\
57 \\
124 \\
50 \\
42 \\
159 \\
197 \\
40 \\
138\end{array}$ & $\begin{array}{r}486,870 \\
402,567 \\
171,300 \\
707,026 \\
1,929,177 \\
312,945 \\
613,524 \\
72,000 \\
141,768 \\
746,951 \\
934,164 \\
114,282 \\
598,540\end{array}$ & $\begin{array}{r}\$ 140,969,005 \\
108,528,726 \\
89,746,992 \\
246,985,034 \\
489,481,061 \\
12,807,337 \\
95,874,327 \\
33,740,309 \\
209,199,893 \\
510,109,742 \\
1,346,487,097 \\
111,660,685 \\
945,064,773\end{array}$ & $\begin{array}{l}1971 \\
1972 \\
1973 \\
1974 \\
1975 \\
1976 \\
1977 \\
1978 \\
1979 \\
1980 \\
1981 \\
1982 \\
1983\end{array}$ & $\begin{array}{l}1 \\
2 \\
2 \\
4 \\
4 \\
4 \\
2 \\
4 \\
6 \\
3 \\
7 \\
5 \\
7 \\
\end{array}$ & $\begin{array}{r}11 \\
178 \\
187 \\
356 \\
321 \\
246 \\
211 \\
249 \\
351 \\
218 \\
425 \\
357 \\
1,251 \\
\end{array}$ & $\begin{array}{r}37,222 \\
826,195 \\
1,032,570 \\
1,762,158 \\
1,679,877 \\
1,277,937 \\
1,100,734 \\
1,297,274 \\
1,767,443 \\
1,134,238 \\
2,237,005 \\
1,886,360 \\
6,593,506 \\
\end{array}$ & $\begin{array}{r}96,304,523 \\
2,251,347,556 \\
3,082,462,611 \\
5,022,860,815 \\
1,088,133,152 \\
2,242,898,467 \\
1,568,564,745 \\
1,767,042,064 \\
5,078,861,692 \\
4,204,640,257 \\
6,602,665,712 \\
3,987,490,009 \\
5,749,016,369\end{array}$ \\
\hline 07 & & 10 & and 1969 & sales & TOTAL & $: 79$ & 6,013 & $29,863,633$ & $\$ 47,082,942,953$ \\
\hline
\end{tabular}
each included one sulfur lease; the 1960 and 1967 sales each Included one salt lease. 


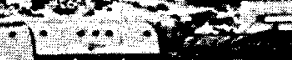
$1=1$

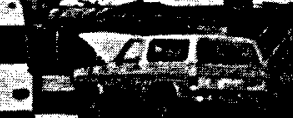




\section{ONSHORE FEDERAL MINERAL REVENUES}

In spite of a 15 percent drop in onshore gas production, royalty revenues from those Federal leases declined less than 1 percent in 1983. The main offsetting factor was a substantial increase in market value--up from $\$ 2.40$ to $\$ 2.83$ per Mcf. Coal and oil revenues, affected by royalty rates and market declines respectively, were also down slightly. But production for both edged upwards. Other solid and fluid mineral revenues rose 9 percent. Thus, onshore Federal revenues declined less than 1 percent overall--from $\$ 972.5$ million in 1982 to $\$ 967.9$ million in 1983 .

Oil and coal production were up slightly--1 million barrels and 4.4 million tons. Nevertheless, lower market values brought lower royalty revenues for both commodities.

Oil production increased less than 1 percent: from 150 to 151 million barrels. Following world trends, its market value decreased somewhat, however. Thus, oil royalties were down from $\$ 525$ to $\$ 523$ million. Gas royal ties were also down slightly--from $\$ 349.4$ to $\$ 348.6$ million.

Coal production increased from 101 million tons to 105 million tons, but royalty revenues dropped--from $\$ 61.9$ million to $\$ 56.7$ million. This decline in coal royalty revenues resulted in part from increased production of lower value coal, as well as from improved extraction from older leases where royalty rates are still calculated on a cents-per-ton basis.

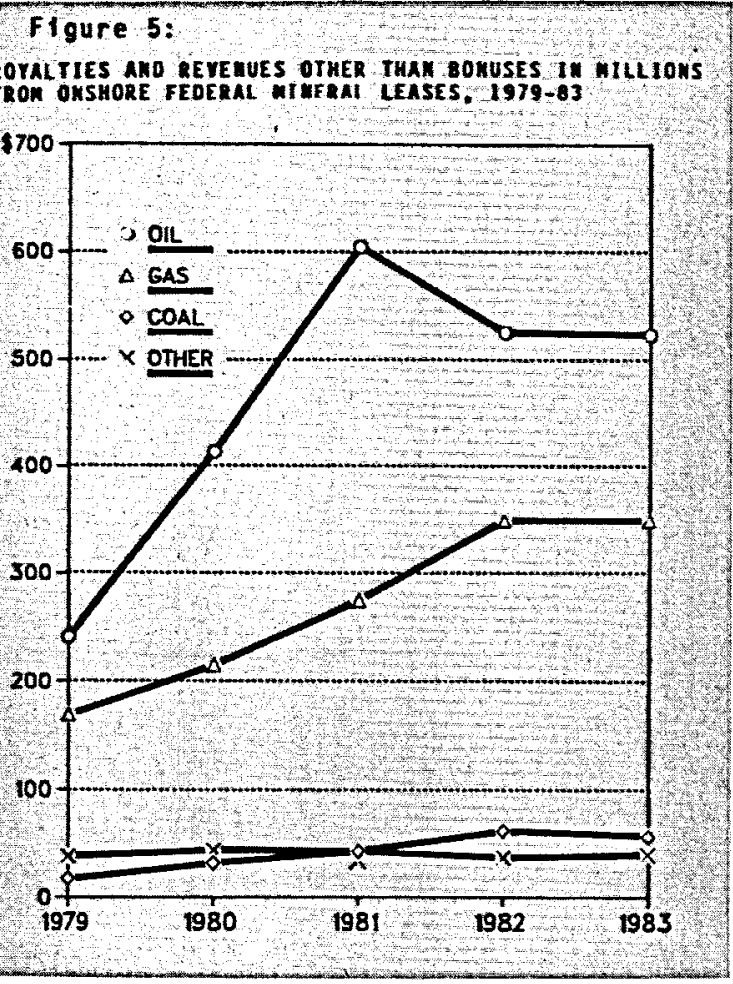

Royalty revenues from fluid and solid minerals other than oil, gas, and coal were up in 1983-from the previous year's total of $\$ 36,613,331$ to $\$ 39,906,641$ (Table 11, page 34).

Competitive mineral leasing activity slowed in the onshore Federal lands-601 leases in 305,396 acres as compared to 645 in 1.3 million acres in 1982. Bonus bids totalled $\$ 62,108,205$ (Tables 12 \& 13). Another 12,349 mineral leases were issued noncompetitively in 1983. 
Oil and gas leasing activity and bonus revenues were strongest in New Mexico (224 leases issued for 44,604 acres, $\$ 14,599,475)$, Alaska (2, for 23,133 acres, $\$ 5,723,850)$, Utah $(51$, for 18,273 acres, $\$ 4,617,317)$, and Wyoming (93, for 18,173 acres, $\$ 3,397,045$ ). But the largest onshore bonuses of $\$ 23,689,632$ were paid for the 2 coal leases issued for 5,176 acres in Wyoming (Table 12).

Geothermal leases continued to interest industry also. In 3 States, 85 leases covering 163,277 acres were issued in 1983. These accounted for a total of $\$ 2,145,376$ in bonus bids, with 67 leases in Oregon covering 138,577 acres and bringing in $\$ 2,141,097$ of that amount alone.

Leases in Wyoming and New Mexico again dominated onshore production (Table 11). Minerals produced in Wyoming had a market value of $\$ 3.8$ billion with royalties of $\$ 375.3$ million, while those produced in New Mexico were valued at $\$ 2.3$ billion with royal ties of $\$ 264.1$ million.

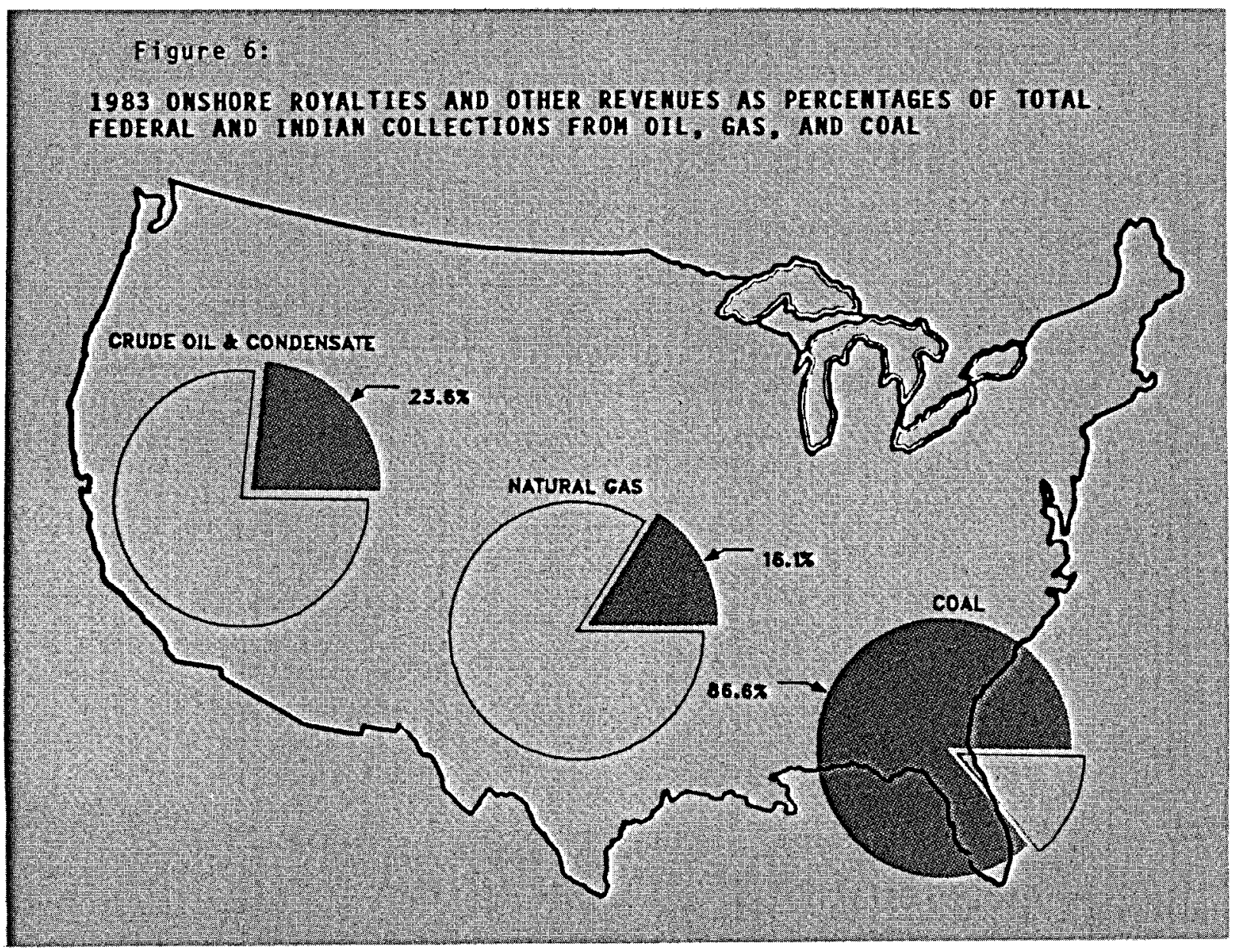


Table 11:

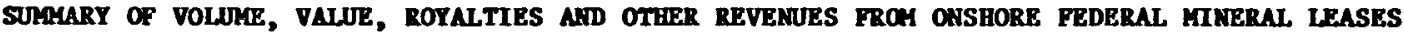
BY STATE AND COAMODIT, 1920-83

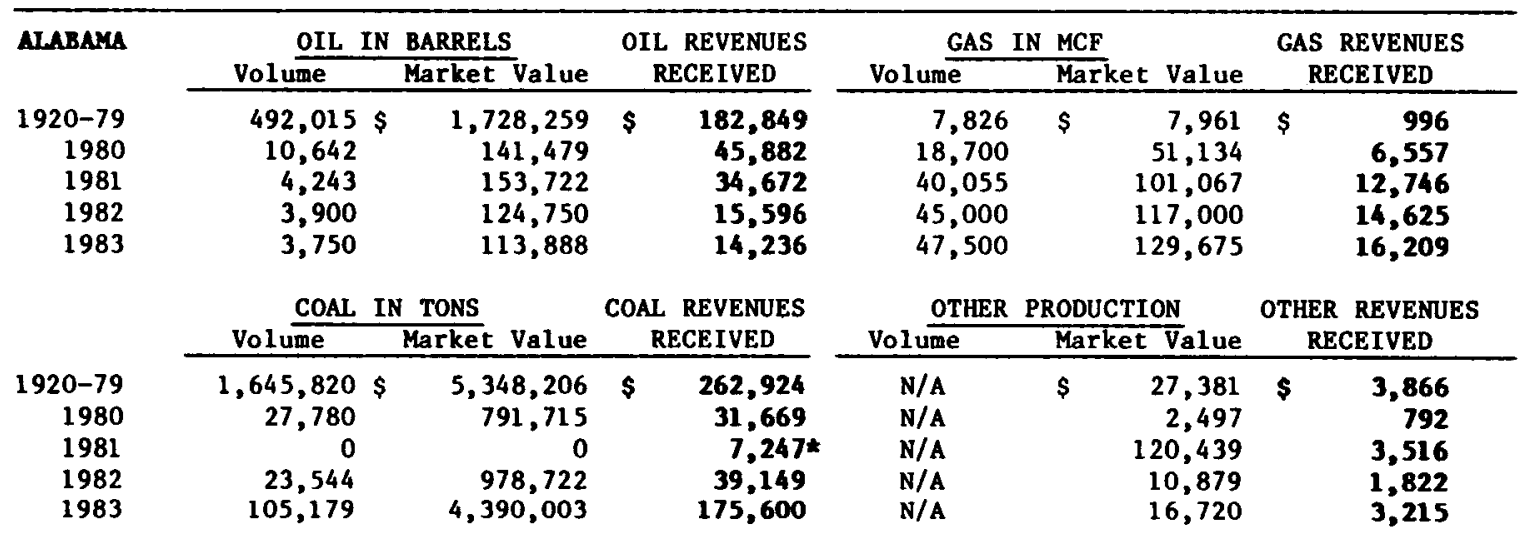

*Advance royalties paid.

TOTAL REVENUES

\begin{tabular}{rrr}
\multicolumn{2}{c}{ ALL } & MINERALS \\
\hline $1920-79$ & $\$$ & 450,635 \\
1980 & & 84,900 \\
1981 & & 58,181 \\
1982 & & 71,242 \\
1983 & & 209,260 \\
$1920-83$ & $\$$ & 874,218 \\
\end{tabular}

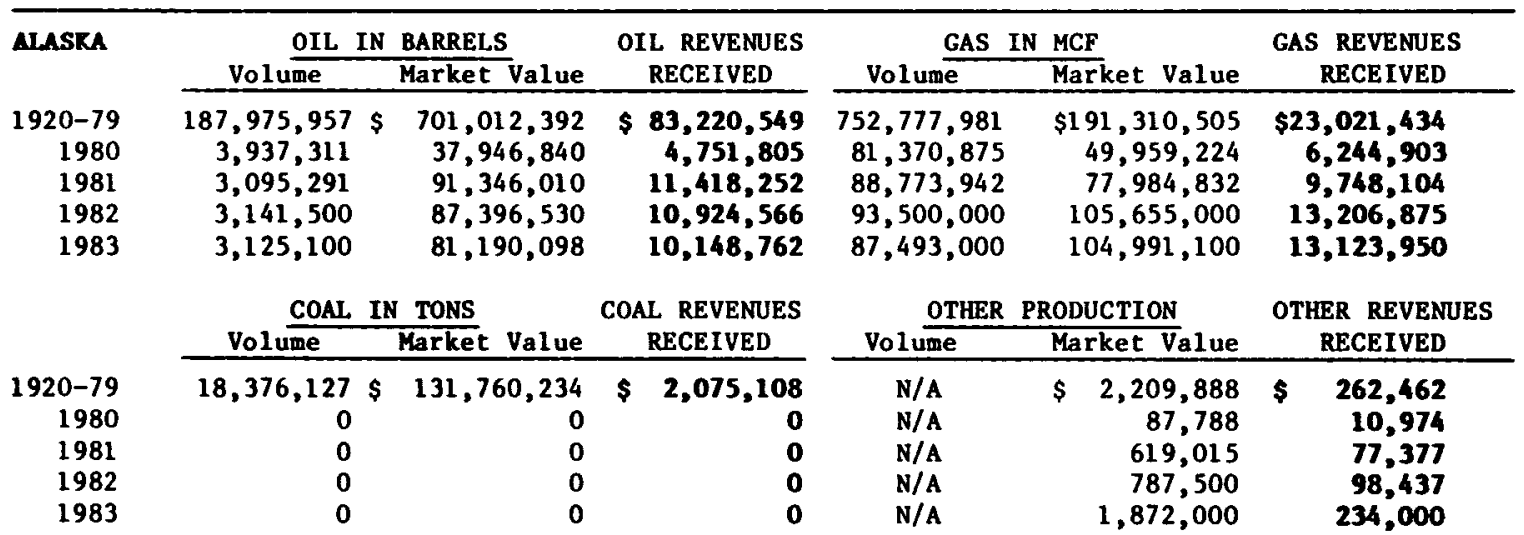

TOTAL REVENUES

ALL MINERALS

\begin{tabular}{rr}
$1920-79$ & $\$ 108,579,553$ \\
1980 & $11,007,682$ \\
1981 & $21,243,733$ \\
1982 & $24,229,878$ \\
1983 & $23,506,712$ \\
$1920-83$ & $\$ 188,567,558$ \\
\hline
\end{tabular}

NOTE: 011 volyme in barrels ( 42 U.S. gallons), gas in thousands of cublc feet (Mcf), coal in short tons $(\$ 2,000)$, revenues in dollars. "Other" includes certain of the solid and fluid minerals footnoted in Table 2 . 
Table 11 (cont'd):

SUMAARY OF VOLDHE, VALUE, ROTALTIES AND OTHER REVENUES FROA ONSHORE FEDERAL MINERAL IEASES BY STATE AND CONODITY, 1920-83

\begin{tabular}{|c|c|c|c|c|c|c|c|}
\hline \multirow[t]{2}{*}{ ARIZONA } & GAS & IN MCF & & GAS REVENUES & OTHER & PRODUCTION & \multirow{2}{*}{$\begin{array}{l}\text { OTHER REVENUES } \\
\text { RECEIVED }\end{array}$} \\
\hline & Volume & Market & Value & RECEIVED & Volume & Market Value & \\
\hline $\begin{array}{r}1920-79 \\
1983\end{array}$ & $\begin{array}{r}124,822 \\
0\end{array}$ & $\$$ & $\begin{array}{r}62,572 \\
0\end{array}$ & $\$ 37,551$ & $\begin{array}{l}\text { N/A } \\
\text { N/A }\end{array}$ & $\begin{array}{r}8,00.4,634 \\
0\end{array}$ & $\begin{array}{r}519,428 \\
0\end{array}$ \\
\hline
\end{tabular}

TOTAL REVENUES

ALL MTNERALS

\begin{tabular}{rrr}
\hline $1920-79$ & $\$$ & 556,979 \\
$1980-83$ & & 0 \\
$1920-83$ & $\$$ & 556,979 \\
\hline
\end{tabular}

\begin{tabular}{|c|c|c|c|c|c|c|c|c|}
\hline ARRAMSAS & OIL IN & BARRELS & $\frac{\mathrm{LS}}{\mathrm{t} \text { Value }}$ & $\begin{array}{l}\text { OIL REVENUES } \\
\text { RECEIVED }\end{array}$ & \multicolumn{2}{|c|}{ GAS IN MCF } & Value & $\begin{array}{l}\text { GAS REVENUES } \\
\text { RECEIVED }\end{array}$ \\
\hline $\begin{array}{r}1920-79 \\
1980 \\
1981 \\
1982 \\
1983\end{array}$ & $\begin{array}{r}47,634 \\
478 \\
225 \\
329 \\
450\end{array}$ & 1 & $\begin{array}{r}127,261 \\
7,257 \\
16,344 \\
11,542 \\
12,825\end{array}$ & $\begin{array}{r}18,902 \\
1,209 \\
2,290 \\
1,443 \\
1,603\end{array}$ & $\begin{array}{r}38,225,971 \\
4,935,402 \\
5,328,022 \\
5,044,312 \\
4,750,000\end{array}$ & $\begin{array}{r}23,7 \\
8,6 \\
7,6 \\
11,6 \\
12,6\end{array}$ & $\begin{array}{l}39,257 \\
35,188 \\
10,781 \\
82,636 \\
04,600\end{array}$ & $\begin{array}{r}\$ 3,388,949 \\
1,237,931 \\
1,012,211 \\
1,622,411 \\
1,575,575\end{array}$ \\
\hline
\end{tabular}

\begin{tabular}{|c|c|c|c|c|c|c|}
\hline \multirow[b]{2}{*}{$\begin{array}{r}1920-79 \\
1980 \\
1981 \\
1982 \\
1983\end{array}$} & $\begin{array}{c}\text { Volume } \\
\frac{\text { OTHER }}{\text { N/A }} \\
\text { N/A }\end{array}$ & $\frac{\text { PRODUCTION }}{\text { Market Value }}$ & $\begin{array}{l}\text { OTHER REVENUES } \\
\text { RECEIVED }\end{array}$ & \multicolumn{3}{|c|}{$\begin{array}{c}\text { TOTAL REVENUES } \\
\text { ALL MINERALS }\end{array}$} \\
\hline & $\begin{array}{l}\text { N/A } \\
\text { N/A } \\
\text { N/A } \\
\text { N/A } \\
\text { N/A }\end{array}$ & $\begin{array}{c}\$ 1,741,590 \\
(42,811) * \\
8,415 \\
11,992 \\
5,857\end{array}$ & $\begin{array}{c}\$ 68,106 \\
(5,673) * \\
260 \\
600 \\
293\end{array}$ & $\begin{array}{r}1920-79 \\
1980 \\
1981 \\
1982 \\
1983 \\
1920-83\end{array}$ & $\$$ & $\begin{array}{l}3,475,957 \\
1,233,467 \\
1,014,761 \\
1,624,454 \\
1,577,471 \\
8,926,110\end{array}$ \\
\hline
\end{tabular}

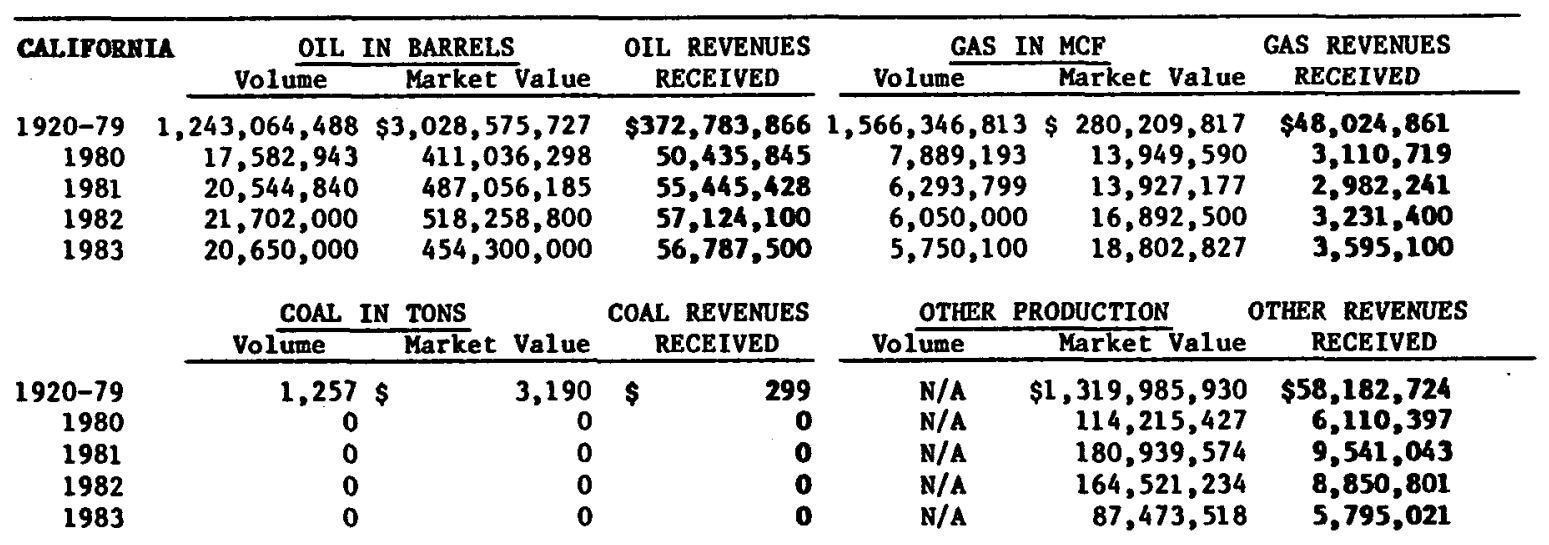

TOTAL REVENUES

ALL MINERALS

\begin{tabular}{rr}
\hline $1920-79$ & $\$ 478,991,750$ \\
1980 & $59,656,961$ \\
1981 & $67,968,712$ \\
1982 & $69,206,301$ \\
1983 & $66,177,621$ \\
$1920-83$ & $\$ 742,001,345$ \\
\hline
\end{tabular}


Table 11 (cont'd):

SUMARY Of VOLOME, VALU, ROYALTIES AND OTHER REVENUES FROA OASHORE FEDERAL MTMERAL IRASES EY STATE AND COEODITY, 1920-83

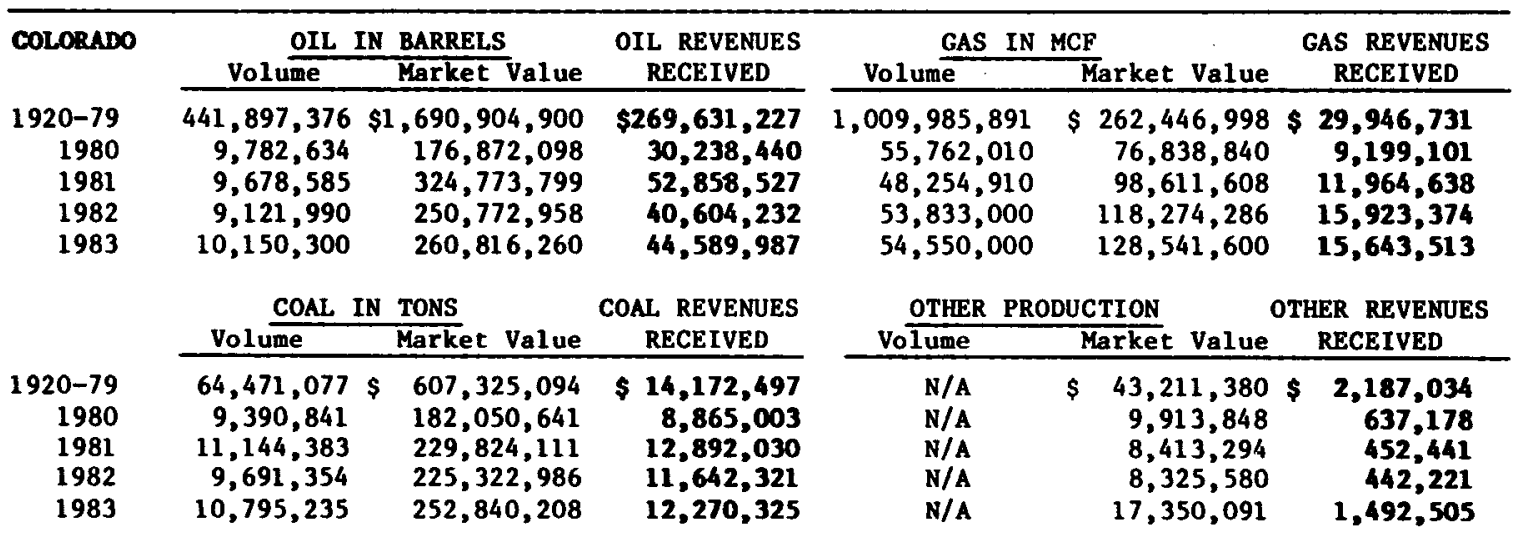

TOTAL REVENUES

ALL MINERALS

\begin{tabular}{rr}
\hline $1920-79$ & $\$ 315,937,489$ \\
1980 & $48,939,722$ \\
1981 & $78,167,636$ \\
1982 & $68,612,148$ \\
1983 & $73,996,330$ \\
$1920-83$ & $\$ 585,653,325$ \\
\hline
\end{tabular}

\begin{tabular}{|c|c|c|c|c|c|c|c|}
\hline FLORIDA & Volume $\frac{\text { OIL IN }}{\text { ume }}$ & $\frac{\text { BARRELS }}{\text { Market }}$ & $\frac{s}{\text { Value }}$ & $\begin{array}{l}\text { OIL REVENUES } \\
\text { RECEIVED }\end{array}$ & Volume & $\frac{\text { PRODUCTION }}{\text { Market Value }}$ & $\begin{array}{c}\text { OTHER REVENUES } \\
\text { RECEIVED }\end{array}$ \\
\hline $\begin{array}{r}1920-79 \\
1980 \\
1981 \\
1982 \\
1983\end{array}$ & $\begin{array}{r}7,256 \\
910 \\
0 \\
0 \\
0\end{array}$ & $\$$ & $\begin{array}{r}90,418 \\
16,865 \\
0 \\
0 \\
0\end{array}$ & $\begin{array}{r}\$ 11,303 \\
2,108 \\
0 \\
0 \\
0\end{array}$ & $\begin{array}{l}\text { N/A } \\
\text { N/A } \\
\text { N/A } \\
\text { N/A } \\
\text { N/A }\end{array}$ & $\begin{array}{r}342,263 \\
2,249,442 \\
4,135,528 \\
2,925,038 \\
8,160,555\end{array}$ & $\begin{array}{r}20,267 \\
113,749 \\
209,133 \\
146,252 \\
411,089\end{array}$ \\
\hline
\end{tabular}

TOTAL REVENUES

ALL MINERALS

\begin{tabular}{rrr}
\hline $1920-79$ & $\$$ & 31,570 \\
1980 & & 115,857 \\
1981 & & 209,133 \\
1982 & & 146,252 \\
1983 & & 411,089 \\
$1920-83$ & $\$ \quad 913,901$ \\
\hline
\end{tabular}

\begin{tabular}{|c|c|c|c|c|c|c|}
\hline GEORGIA & Volume & $\frac{\text { PRODUCTION }}{\text { Market Value }}$ & $\begin{array}{c}\text { OTHER REVENUES } \\
\text { RECEIVED } \\
\end{array}$ & \multicolumn{3}{|c|}{$\begin{array}{c}\text { TOTAL REVENUES } \\
\text { ALL MINERALS }\end{array}$} \\
\hline $\begin{array}{r}1920-79 \\
1980 \\
1981 \\
1982 \\
1983\end{array}$ & $\begin{array}{l}\text { N/A } \\
\text { N/A } \\
\text { N/A } \\
\text { N/A } \\
\text { N/A }\end{array}$ & $\begin{array}{r}\$ 2,705,921 \\
204,373 \\
190,070 \\
71,156 \\
8,044\end{array}$ & $\begin{array}{r}\$ 99,013 \\
9,462 \\
9,962 \\
3,188 \\
340\end{array}$ & $\begin{array}{r}1920-79 \\
1980 \\
1981 \\
1982 \\
1983\end{array}$ & $\$$ & $\begin{array}{r}99,013 \\
9,462 \\
9,962 \\
3,188 \\
340\end{array}$ \\
\hline & & & & $1920-83$ & $\$$ & 121,965 \\
\hline
\end{tabular}


Table 11 (cont'd):

SURAMRY OF VOLURR, VAUUE, ROTALTIES AKD OTHER REVENUES FROA ONSHORE FEDERAL MIHERAL LEASES BY STATE AND COMODITY, 1920-83

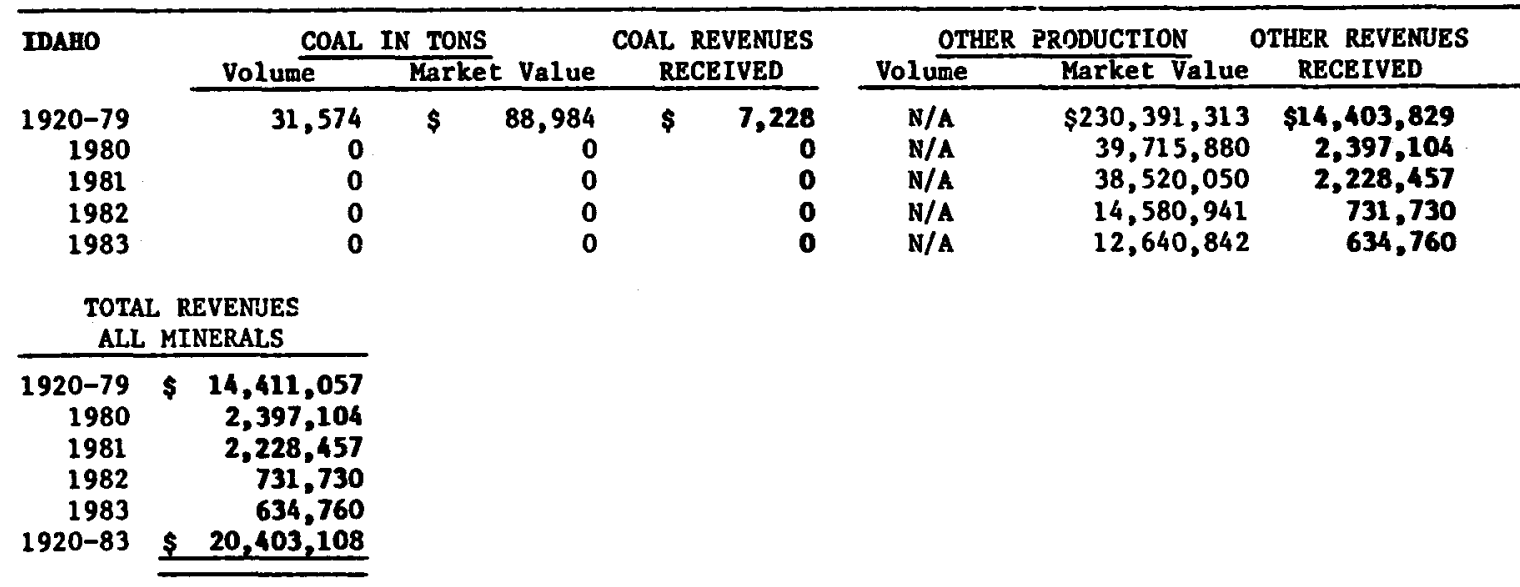

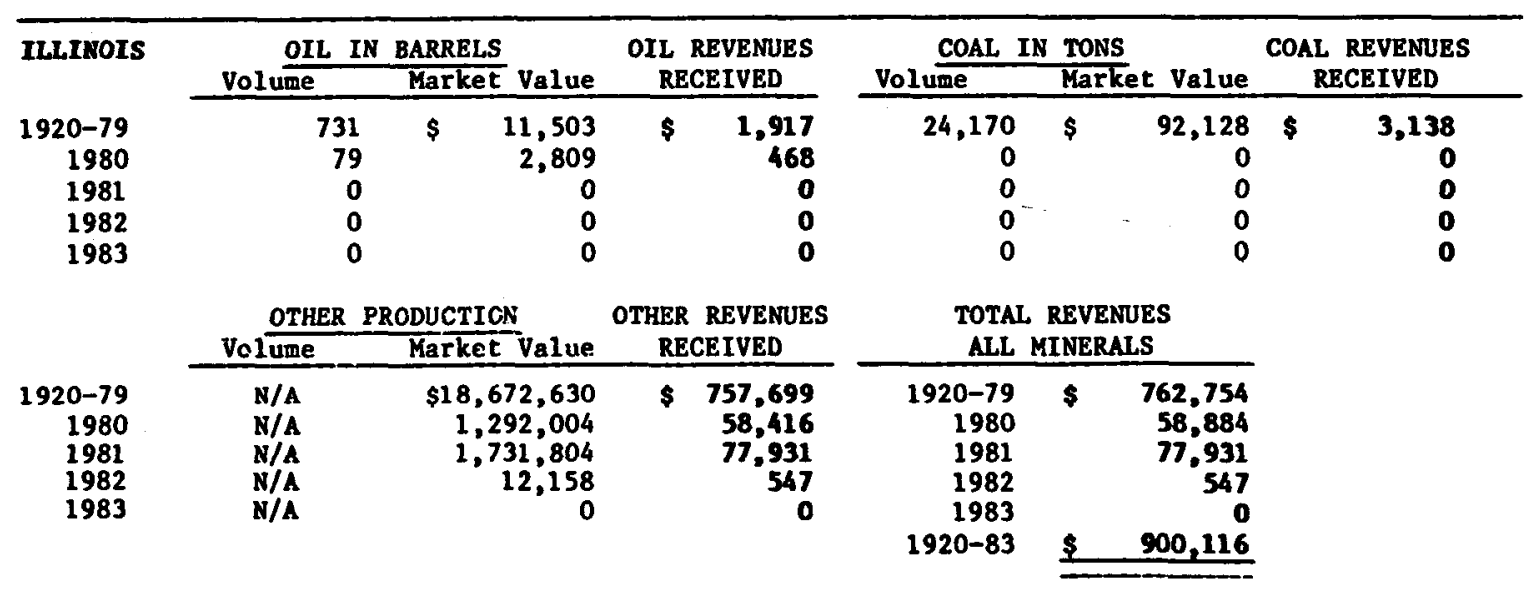

\begin{tabular}{|c|c|c|c|c|c|c|c|c|}
\hline \multirow{2}{*}{$\begin{array}{r}\text { INDIAKA } \\
1920-79 \\
1983\end{array}$} & \multirow{2}{*}{$\frac{\text { OIL IN }}{\text { Volume }}$} & \multicolumn{2}{|c|}{$\frac{\text { BARRELS }}{\text { Market Value }}$} & \multicolumn{2}{|c|}{$\begin{array}{c}\text { OIL REVENUES } \\
\text { RECEIVED } \\
\end{array}$} & \multicolumn{3}{|c|}{$\begin{array}{c}\text { TOTAL REVENUES } \\
\text { ALL MINERALS }\end{array}$} \\
\hline & & $\mathbf{\$}$ & $\begin{array}{r}15,529 \\
0\end{array}$ & $\$$ & $\begin{array}{r}1,859 \\
0\end{array}$ & $\begin{array}{l}1920-79 \\
1980-83 \\
1920-83\end{array}$ & $\$$ & $\begin{array}{r}1,859 \\
0 \\
1,859 \\
\end{array}$ \\
\hline
\end{tabular}


Table 11 (cont'd):

SULAary OF VOLUEE, VALUE, ROTALTIES AKD OTEER REVENUES FROA ONSHORE FEDERAL MINERAI LEASES BT STATE AND coniodT, 1920-83

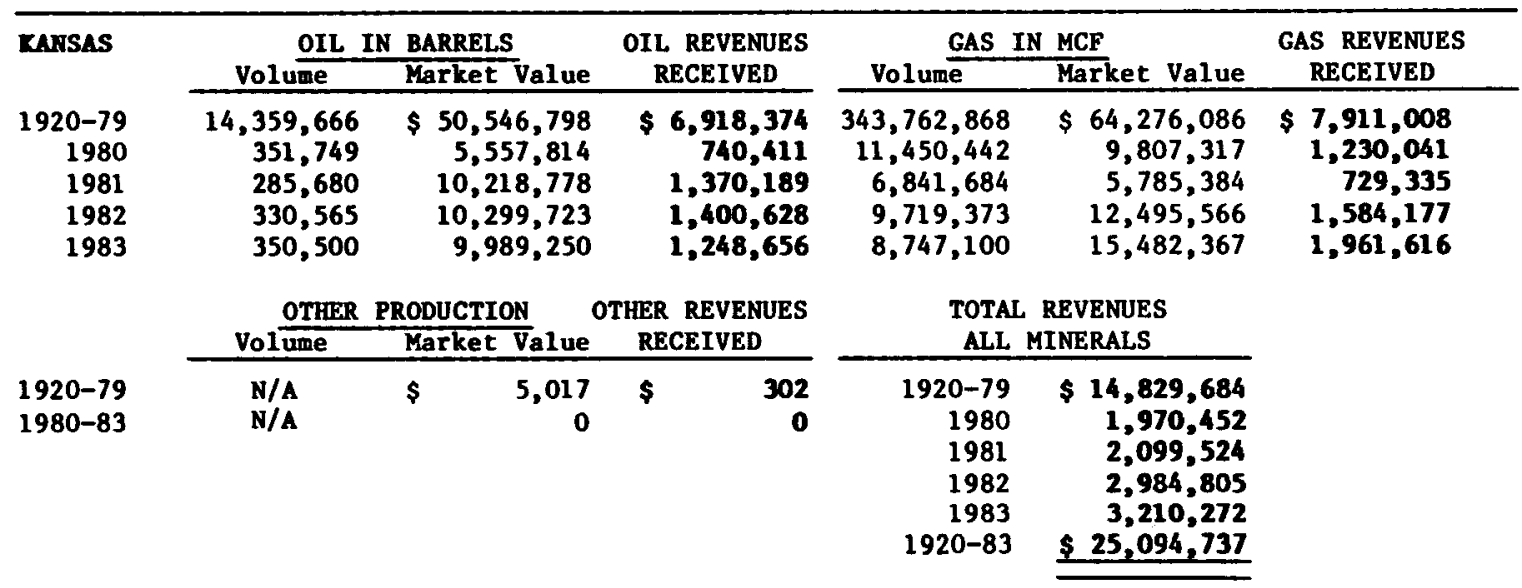

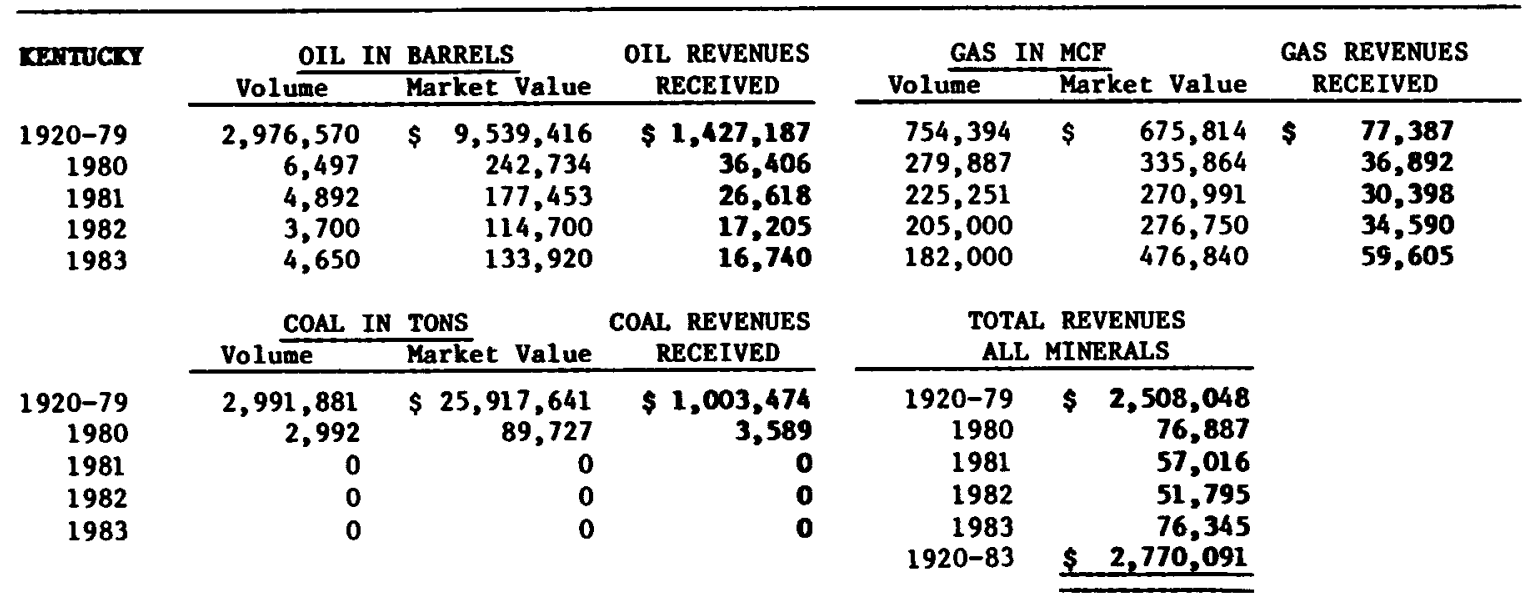

\begin{tabular}{|c|c|c|c|c|}
\hline \multirow[t]{2}{*}{ LOUISIARA } & OIL IN & BARRELS & OIL REVENUES & $\frac{\text { GAS IN MCF }}{\text { Market Value }}$ \\
\hline & Volume & Market Value & RECEIVED & Volume Market Value \\
\hline \multirow[t]{2}{*}{$\begin{array}{r}1920-79 \\
1980 \\
1981 \\
1982 \\
1983\end{array}$} & $\begin{array}{r}145,655,048 \\
2,054,703 \\
1,464,854 \\
1,734,145 \\
2,004,500\end{array}$ & $\begin{array}{r}\$ 510,067,485 \\
30,427,595 \\
51,622,050 \\
45,220,999 \\
59,132,750\end{array}$ & $\begin{array}{r}\$ 60,658,840 \\
3,583,097 \\
5,686,906 \\
5,913,809 \\
7,391,594\end{array}$ & $\begin{array}{rr}1,397,105,638 & \$ 257,962,007 \\
17,955,861 & 14,666,618 \\
15,512,606 & 13,040,634 \\
17,177,498 & 19,944,754 \\
15,459,300 & 23,807,322\end{array}$ \\
\hline & Volume & $\frac{\text { ODUCTION }}{\text { Market Value }}$ & $\begin{array}{c}\text { OTHER REVENUES } \\
\text { RECEIVED } \\
\end{array}$ & $\begin{array}{l}\text { TOTAL REVENUES } \\
\text { ALL MINERALS }\end{array}$ \\
\hline $\begin{array}{r}1920-79 \\
1980 \\
1981 \\
1982 \\
1983\end{array}$ & $\begin{array}{l}\text { N/A } \\
\text { N/A } \\
\text { N/A } \\
\text { N/A } \\
\text { N/A }\end{array}$ & $\begin{array}{r}\$ 40,622,159 \\
5,788,422 \\
7,024,299 \\
7,194,180 \\
6,330,000\end{array}$ & $\begin{array}{r}6,609,649 \\
912,874 \\
1,072,336 \\
1,026,086 \\
902,800\end{array}$ & $\begin{array}{rr}1920-79 & \$ 104,658,722 \\
1980 & 6,428,773 \\
1981 & 8,521,168 \\
1982 & 9,539,013 \\
1983 & 11,396,488 \\
1920-83 & \$ 140,544,164 \\
\end{array}$ \\
\hline
\end{tabular}


Table 11 (cont'd):

SUMART OF VOLOAE, VALUE, ROTALTIES AMD OTHER REVEAUES FROA OASHORE FEDERAL MIMERAL LEASES BY STATE AMD COAHODIT, i920-83

\begin{tabular}{|c|c|c|c|c|c|c|c|c|}
\hline \multirow{2}{*}{$\begin{array}{r}\text { MAINE } \\
1920-79 \\
1983\end{array}$} & \multirow{2}{*}{$\begin{array}{c}\frac{\text { OTHER }}{\text { Volume }} \\
N / A \\
N / A\end{array}$} & \multicolumn{2}{|c|}{ PRODUCTION } & \multicolumn{2}{|c|}{$\begin{array}{c}\text { OTHER REVENUES } \\
\text { e RECEIVED } \\
\end{array}$} & \multicolumn{3}{|c|}{$\begin{array}{c}\text { TOTAL REVENUES } \\
\text { ALL MINERALS }\end{array}$} \\
\hline & & $\$$ & $\begin{array}{r}9,711 \\
0\end{array}$ & $\$$ & $\begin{array}{r}818 \\
0\end{array}$ & $\begin{array}{l}1920-79 \\
1980-83 \\
1920-83\end{array}$ & $\$$ & $\begin{array}{r}818 \\
0 \\
818 \\
\end{array}$ \\
\hline
\end{tabular}

\begin{tabular}{|c|c|c|c|c|c|c|c|c|}
\hline \multirow{2}{*}{$\begin{array}{r}\text { KARTLAKD } \\
1920-79 \\
1980 \\
1981 \\
1982 \\
1983\end{array}$} & \multicolumn{2}{|c|}{$\begin{array}{l}\text { GAS PRODUCTION } \\
\text { Volume Market }\end{array}$} & ken value & \multicolumn{2}{|c|}{$\begin{array}{c}\text { GAS REVENUES } \\
\text { RECEIVED } \\
\end{array}$} & \multicolumn{3}{|c|}{$\begin{array}{c}\text { TOTAL REVENUES } \\
\text { ALL MINERALS }\end{array}$} \\
\hline & $\begin{array}{r}3,800,246 \\
4,482 \\
2,557 \\
2,400 \\
2,160\end{array}$ & $\$$ & $\begin{array}{r}1,033,644 \\
9,905 \\
4,952 \\
4,992 \\
5,530\end{array}$ & $\mathbf{\$}$ & $\begin{array}{r}129,946 \\
1,238 \\
619 \\
624 \\
691\end{array}$ & $\begin{array}{r}1920-79 \\
1980 \\
1981 \\
1982 \\
1983 \\
1920-83\end{array}$ & $\$$ & $\begin{array}{r}129,946 \\
1,238 \\
619 \\
624 \\
691 \\
133,118\end{array}$ \\
\hline
\end{tabular}

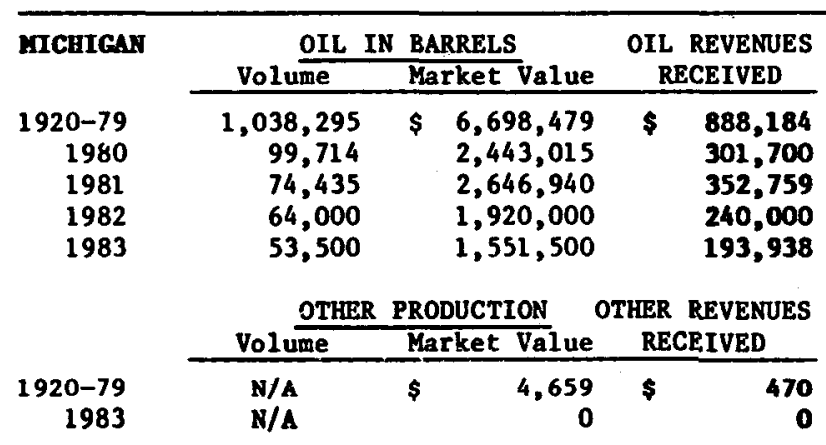

\begin{tabular}{crc}
\multicolumn{2}{c}{ GAS IN MCF } & \multicolumn{2}{c}{ GAS REVENUES } \\
Volume & Narket Value & RECEIVED \\
\hline $4,038,899$ & $\$ 3,313,778$ & $\$ 470,717$ \\
594,958 & 946,864 & 113,029 \\
575,628 & $1,681,725$ & 210,483 \\
560,000 & $1,708,000$ & 213,500 \\
498,552 & $1,691,472$ & 211,428
\end{tabular}

TOTAL REVENUES

ALL MINERALS

\begin{tabular}{rr}
$1920-79$ & $\$ 1,359,371$ \\
1980 & 414,729 \\
1981 & 563,242 \\
1982 & 453,500 \\
1983 & 405,366 \\
$1920-83$ & $\$ 3,196,208$ \\
\hline
\end{tabular}

\begin{tabular}{|c|c|c|c|c|c|c|}
\hline \multirow{2}{*}{$\begin{array}{c}\text { MTnRESOTA } \\
1920-79 \\
1980 \\
1981 \\
1982 \\
1983\end{array}$} & \multirow{2}{*}{$\begin{array}{l}\text { OTHER } \\
\text { Volume } \\
\text { N/A } \\
\text { N/A } \\
N / A \\
N / A \\
N / A\end{array}$} & $\begin{array}{l}\text { PRODUCTION } \\
\text { Market Value }\end{array}$ & $\begin{array}{c}\text { OTHER REVENUES } \\
\text { RECEIVED } \\
\end{array}$ & \multicolumn{3}{|c|}{$\begin{array}{c}\text { TOTAL REVENUES } \\
\text { ALL MINERALS }\end{array}$} \\
\hline & & $\begin{array}{r}442,883 \\
203,218 \\
3,259,472 \\
32,522 \\
1,292,891\end{array}$ & $\begin{array}{r}16,143 \\
8,129 \\
130,379 \\
3,533 \\
39,185\end{array}$ & $\begin{array}{r}1920-79 \\
1980 \\
1981 \\
1982 \\
1983 \\
1920-83\end{array}$ & $\$$ & $\begin{array}{r}16,143 \\
8,129 \\
130,379 \\
3,533 \\
39,185 \\
197,369 \\
\end{array}$ \\
\hline
\end{tabular}


Table 11 (cont'd):

SURMARY OF VOLUHE, VALUE, ROTALTIES AND OTHER REVENUES FROY ONSHORE FEDERAL MIRERAL LRASES BY STATE AND CONODITY, 1920-83

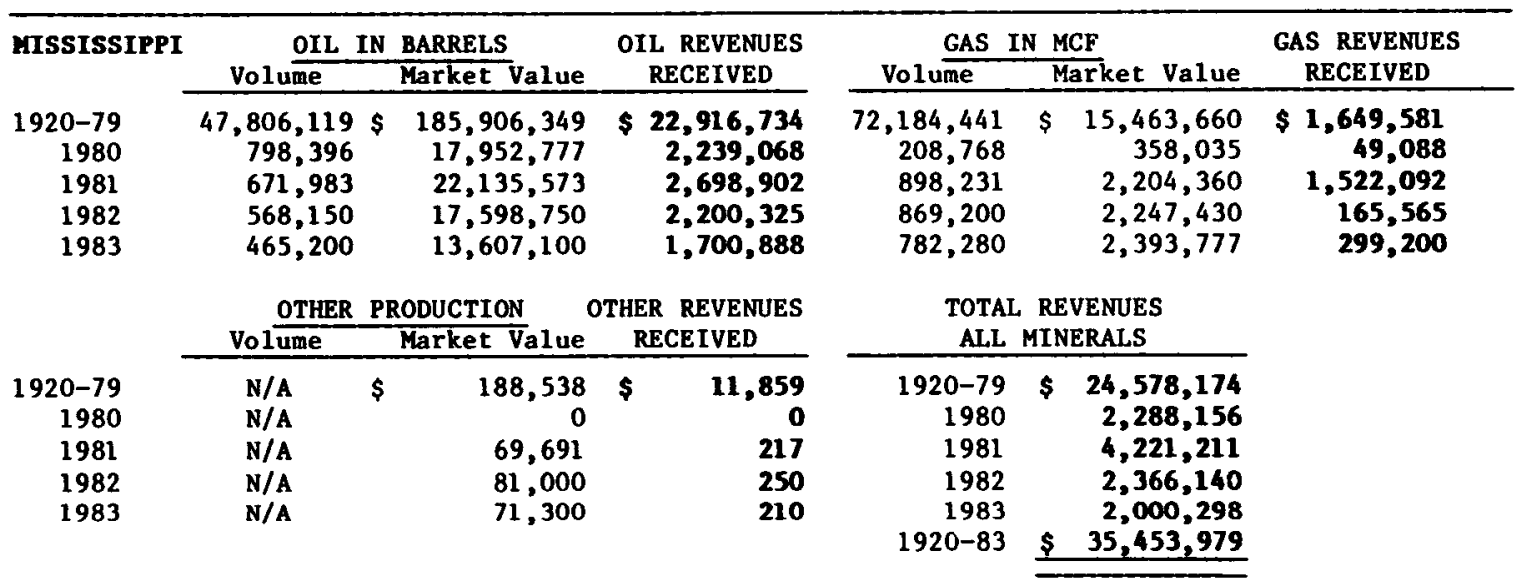

\begin{tabular}{|c|c|c|c|c|c|}
\hline IISSOURI & Volume & $\frac{\text { PRODUCTION }}{\text { Market Value }}$ & $\begin{array}{c}\text { OTHER REVENUES } \\
\text { RECEIVED }\end{array}$ & $\begin{array}{r}\text { TOTA } \\
\text { ALL }\end{array}$ & $\begin{array}{l}\text { REVENUES } \\
\text { MINERALS }\end{array}$ \\
\hline $\begin{array}{r}1920-79 \\
1980 \\
1981 \\
1982 \\
1983\end{array}$ & $\begin{array}{l}\text { N/A } \\
\text { N/A } \\
\text { N/A } \\
\text { N/A } \\
\text { N/A }\end{array}$ & $\begin{array}{r}\$ 1,279,200,857 \\
251,760,373 \\
144,412,821 \\
92,808,570 \\
92,473,768\end{array}$ & $\begin{array}{r}56,985,629 \\
12,588,211 \\
7,169,887 \\
4,652,521 \\
4,617,952\end{array}$ & $\begin{array}{r}1920-79 \\
1980 \\
1981 \\
1982 \\
1983 \\
1920-83\end{array}$ & $\begin{array}{r}\$ 56,985,629 \\
12,588,211 \\
7,169,887 \\
4,652,521 \\
4,617,952 \\
\$ 86,014,200\end{array}$ \\
\hline
\end{tabular}

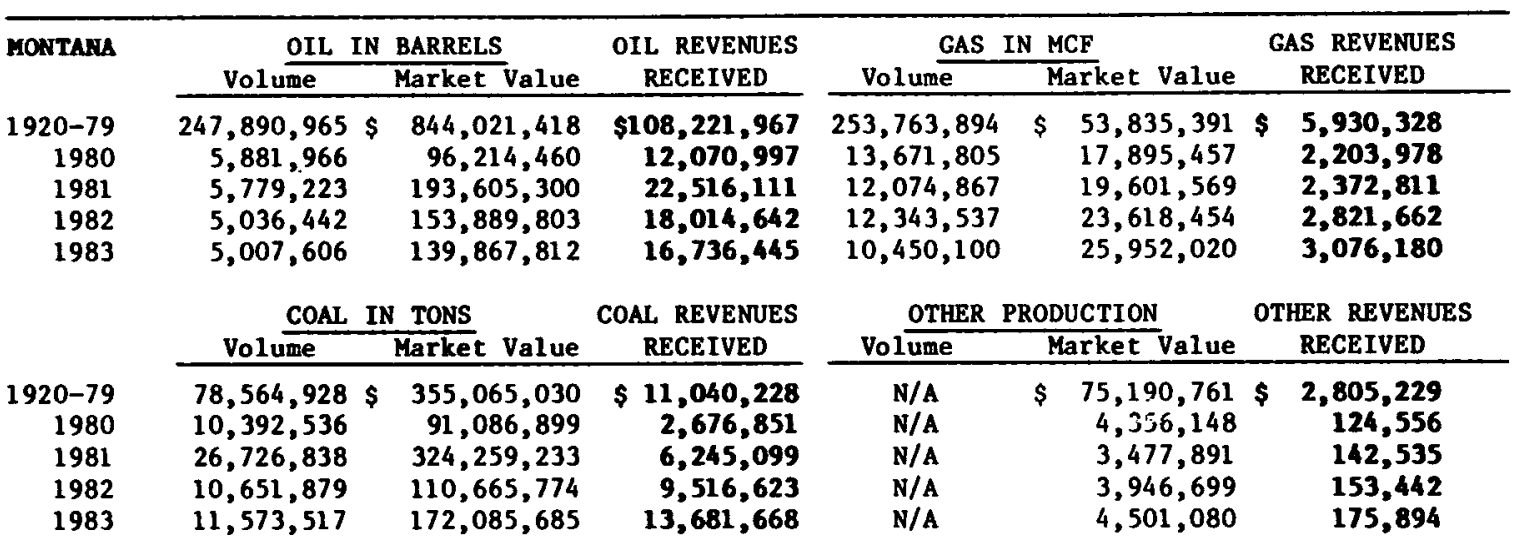

TOTAL REVENUES

ALL MINERALS

\begin{tabular}{rr}
\hline $1920-79$ & $\$ 127,997,752$ \\
1980 & $17,076,382$ \\
1981 & $31,276,556$ \\
1982 & $30,526,369$ \\
1983 & $33,670,187$ \\
$1920-83$ & $\$ 240,547,246$ \\
\cline { 2 - 2 }
\end{tabular} 
Table 11 (cont'd):

SUMMARY OF VOLURE, VALUE, ROYALTIES AND OTHER REVENUES FROA ONSHORE FRDERAL MMKERAL IEASES BT STATE AND COAMODIT, 1920-83

\begin{tabular}{|c|c|c|c|c|c|c|c|c|c|c|}
\hline \multirow{2}{*}{$\begin{array}{r}\text { TEBRASKA } \\
1920-79 \\
1980 \\
1981 \\
1982 \\
1983\end{array}$} & \multirow{2}{*}{\multicolumn{2}{|c|}{$\begin{array}{l}\text { oIL IN } \\
\text { Joiume } \\
830,068 \\
102,853 \\
119,261 \\
108,492 \\
106,064\end{array}$}} & $\frac{\text { BARRELS }}{\text { Market }}$ & \multicolumn{2}{|c|}{$\begin{array}{c}\text { OIL REVENUES } \\
\text { RECEIVED } \\
\end{array}$} & \multicolumn{2}{|c|}{$\begin{array}{c}\text { GAS IN MCF } \\
\text { Volume } \\
\text { Market }\end{array}$} & $\frac{F}{\text { rket Value }}$ & \multicolumn{2}{|c|}{$\begin{array}{l}\text { GAS REVBNUES } \\
\text { RECEIVED } \\
\end{array}$} \\
\hline & & & $\begin{array}{l}6,822,731 \\
3,307,012 \\
4,250,087 \\
3,501,401 \\
3,199,791\end{array}$ & $\$$ & $\begin{array}{l}641,478 \\
513,924 \\
583,363 \\
445,583 \\
308,784\end{array}$ & $\begin{array}{r}8,877,911 \\
438,657 \\
470,739 \\
388,552 \\
359,300\end{array}$ & $\$$ & $\begin{array}{r}4,026,473 \\
662,771 \\
630,792 \\
820,959 \\
525,800\end{array}$ & $\$$ & $\begin{array}{r}665,157 \\
110,462 \\
103,568 \\
136,076 \\
85,900\end{array}$ \\
\hline & Volume & PROD & $\frac{\text { CTION }}{\text { rket Value }}$ & $\begin{array}{r}\text { OTHER } \\
\text { RE }\end{array}$ & $\begin{array}{l}\text { REVENUES } \\
\text { EIVED }\end{array}$ & $\begin{array}{l}\text { TOTAL } \\
\text { ALL }\end{array}$ & $\begin{array}{l}\text { REV } \\
\text { MINE }\end{array}$ & $\begin{array}{l}\text { ENUES } \\
\text { RALS }\end{array}$ & & \\
\hline $\begin{array}{r}1920-79 \\
1980 \\
1981 \\
1982 \\
1983\end{array}$ & $\begin{array}{l}N / A \\
N / A \\
N / A \\
N / A \\
N / A\end{array}$ & $\$$ & $\begin{array}{r}2,633,873 \\
381,417 \\
490,769 \\
421,644 \\
351,000\end{array}$ & , & $\begin{array}{r}227,234 \\
17,166 \\
33,569 \\
28,779 \\
23,500\end{array}$ & $\begin{array}{r}1920-79 \\
1980 \\
1981 \\
1982 \\
1983 \\
1920-83\end{array}$ & $\$$ & $\begin{array}{r}1,533,869 \\
641,552 \\
720,500 \\
610,438 \\
418,184 \\
3,924,543 \\
\end{array}$ & & \\
\hline
\end{tabular}

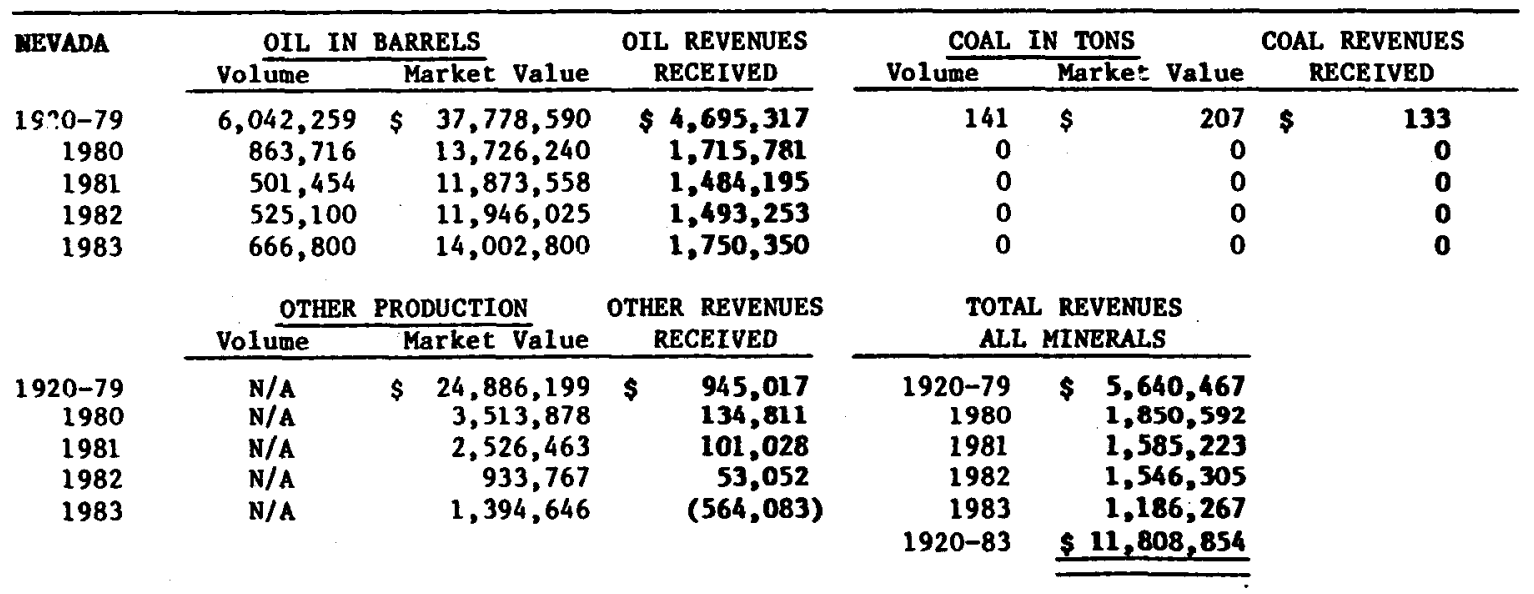


Table 11 (cont'd):

SURMLRY OF VOLOHE, VALUE, ROYALTIES AND OTHER REVENUES FROY ONSHORE FEDERAL MTNERAL IRASES BT STATE AND COHODITY, 1920-83

\begin{tabular}{|c|c|c|c|c|c|c|}
\hline \multirow[t]{2}{*}{ NEW NEXICO } & \multirow{2}{*}{ Volume } & \multirow{2}{*}{$\frac{N \text { BARRELS }}{\text { Market }}$} & \multirow{2}{*}{$\begin{array}{l}\text { OIL REVENUES } \\
\text { RECEIVED } \\
\end{array}$} & \multicolumn{2}{|c|}{ GAS IN MCF } & GAS REVENUES \\
\hline & & & & Volume & Market Value & RECEIVED \\
\hline \multirow[t]{2}{*}{$\begin{array}{r}1920-79 \\
1980 \\
1981 \\
1982 \\
1983\end{array}$} & $\begin{array}{r}985,338,813 \\
21,664,764 \\
21,796,606 \\
18,368,501 \\
17,637,300\end{array}$ & $\begin{array}{r}\$ 3,700,162,705 \\
530,218,728 \\
695,739,128 \\
564,236,227 \\
529,119,000\end{array}$ & $\begin{array}{r}\$ 450,821,729 \\
65,322,151 \\
84,424,779 \\
68,138,838 \\
66,139,875\end{array}$ & $\begin{array}{r}12,935,039,734 \\
584,421,135 \\
648,683,299 \\
687,846,386 \\
510,571,286\end{array}$ & $\begin{array}{r}\$ 3,999,583,957 \\
1,064,844,278 \\
1,341,783,321 \\
1,652,188,050 \\
5 \quad 1,470,445,300\end{array}$ & $\begin{array}{r}\$ 495,278,400 \\
133,031,227 \\
167,916,224 \\
207,174,936 \\
183,805,660\end{array}$ \\
\hline & Volume & $\frac{\text { TONS }}{\text { Market Value }}$ & $\begin{array}{l}\text { COAL REVENUES } \\
\text { RECEIVED }\end{array}$ & Volume & $\frac{\text { PRODUCTION }}{\text { Market Value }}$ & $\begin{array}{l}\text { OTHER REVENUES } \\
\text { RECEIVED }\end{array}$ \\
\hline $\begin{array}{r}1920-79 \\
1980 \\
1981 \\
1982 \\
1983\end{array}$ & $\begin{array}{r}19,798,870 \\
6,316,551 \\
9,184,682 \\
4,403,691 \\
2,290,379\end{array}$ & $\begin{array}{r}173,533,612 \\
81,406,221 \\
100,957,236 \\
86,028,883 \\
41,420,213\end{array}$ & $\begin{array}{r}4,324,887 \\
6,982,294 \\
100,219 \star \\
8,289,584 \\
5,000,506\end{array}$ & $\begin{array}{l}\text { N/A } \\
\text { N/A } \\
\text { N/A } \\
\text { N/A } \\
\text { N/A }\end{array}$ & $\begin{array}{r}\$ 2,997,493,492 \\
297,094,282 \\
289,589,265 \\
338,528,191 \\
254,860,384\end{array}$ & $\begin{array}{r}\$ 124,829,610 \\
12,974,943 \\
10,926,572 \\
12,810,349 \\
9,195,388\end{array}$ \\
\hline
\end{tabular}

TOTAL REVENUES

ALL. MINERALS

\begin{tabular}{rr}
$1920-79$ & $\$ 1,075,254,626$ \\
1980 & $218,310,615$ \\
1981 & $263,367,794$ \\
1982 & $296,413,707$ \\
1983 & $264,141,429$ \\
$1920-83$ & $\$ 2,117,488,171$ \\
\hline
\end{tabular}

\begin{tabular}{|c|c|c|c|c|c|c|c|c|}
\hline \multirow{2}{*}{$\begin{array}{c}\text { WORTH } \\
\text { CAROLIKA } \\
1920-79 \\
1983\end{array}$} & \multirow{2}{*}{$\begin{array}{l}\text { Vol ume } \\
\text { N/A } \\
\text { N/A }\end{array}$} & \multicolumn{2}{|c|}{$\begin{array}{l}\text { PRODUCTION } \\
\text { Market Value }\end{array}$} & \multicolumn{2}{|c|}{$\begin{array}{c}\text { OTHER REVENUES } \\
\text { RECEIVED } \\
\end{array}$} & \multicolumn{3}{|c|}{$\begin{array}{r}\text { TOTAL REVENUES } \\
\text { ALL MINERALS } \\
\end{array}$} \\
\hline & & $\$$ & $\begin{array}{r}315,696 \\
0\end{array}$ & $\$$ & $\begin{array}{r}19,154 \\
0\end{array}$ & $\begin{array}{l}1920-79 \\
1980-83 \\
1920-83\end{array}$ & $\$$ & $\begin{array}{r}19,154 \\
0 \\
19,154 \\
\end{array}$ \\
\hline
\end{tabular}


Table 11 (cont'd):

SURMARY OF VOLUME, VALUE, ROYALTIES AND OTHER REVRKUES FROY ONSHORE FEDERAL MIRERAL LRASES BI STATE AND COMODIT, 1920-83

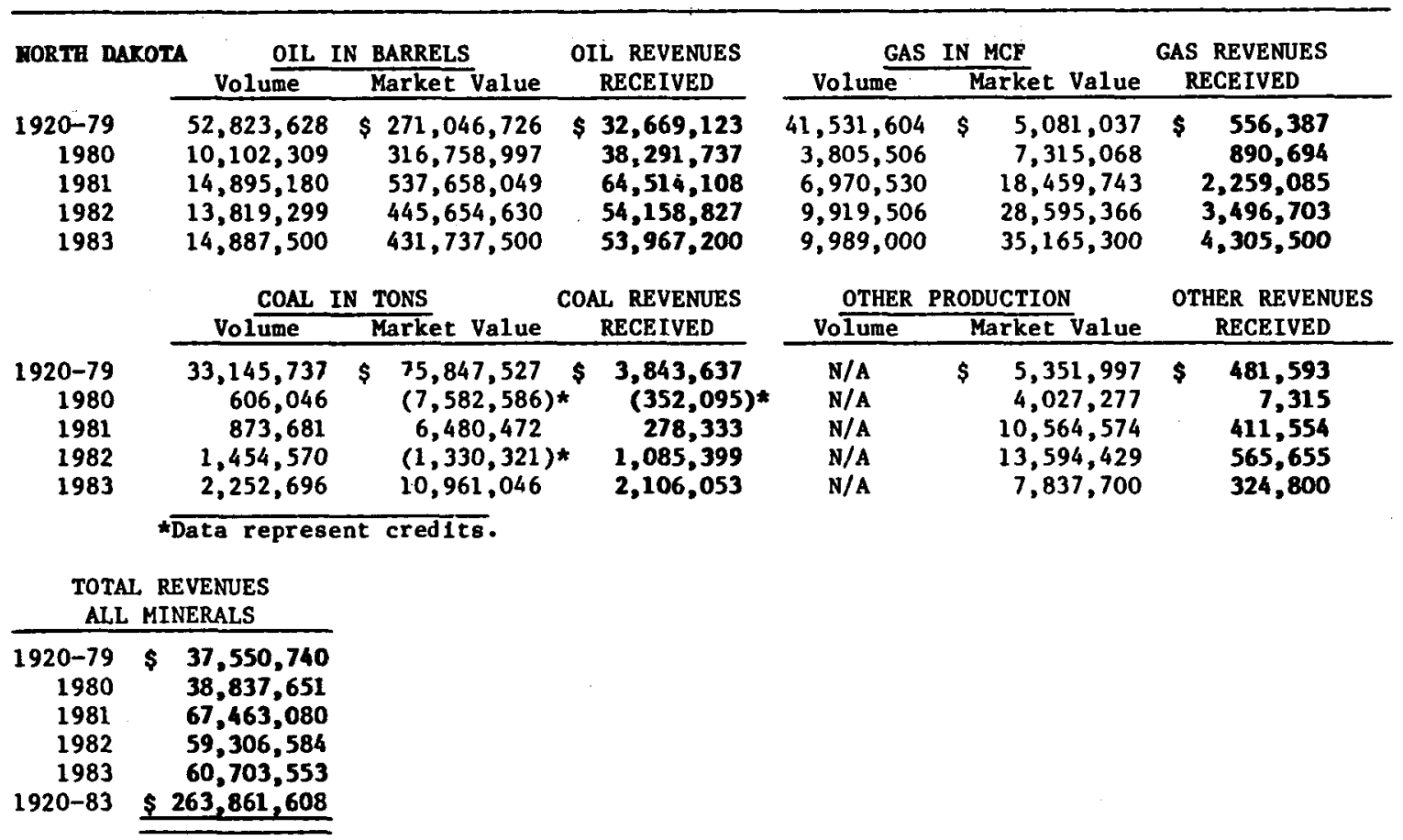

\begin{tabular}{|c|c|c|c|c|c|c|c|c|c|c|}
\hline \multirow{2}{*}{$\begin{array}{l}\text { OHIO } \\
\begin{array}{r}1920-79 \\
1980 \\
1981 \\
1982 \\
1983\end{array}\end{array}$} & \multirow{2}{*}{\multicolumn{2}{|c|}{$\begin{array}{c}\text { OIL IN B } \\
\text { Volume } \\
796,680 \\
4,679 \\
1,648 \\
1,700 \\
1,920\end{array}$}} & $\frac{\text { BARRELS }}{\text { Market Value }}$ & \multicolumn{2}{|c|}{$\begin{array}{c}\text { OIL REVENUES } \\
\text { RECEIVED } \\
\end{array}$} & \multirow{2}{*}{$\begin{array}{c}\text { GAS II } \\
\text { Volume } \\
4,477,291 \\
78,557 \\
50,601 \\
46,600 \\
81,500\end{array}$} & $\frac{\text { MCF }}{\text { Market }}$ & \multicolumn{3}{|c|}{$\begin{array}{c}\text { GAS REVENUES } \\
\text { RECEIVED } \\
\end{array}$} \\
\hline & & & $\begin{array}{r}2,286,555 \\
113,028 \\
57,187 \\
51,000 \\
53,940\end{array}$ & $\mathbf{\$}$ & $\begin{array}{r}353,285 \\
14,121 \\
7,759 \\
6,375 \\
6,732\end{array}$ & & \multicolumn{2}{|c|}{$\begin{array}{r}\$ 1,374,978 \\
67,428 \\
46,320 \\
49,400 \\
89,700\end{array}$} & \multirow[t]{2}{*}{$\$$} & \multirow[t]{3}{*}{$\begin{array}{r}154,050 \\
8,426 \\
5,790 \\
6,170 \\
8,973\end{array}$} \\
\hline & Volume & $\frac{\text { TO }}{\mathbf{M}}$ & $\frac{\mathbf{S}}{\text { rket Value }}$ & COA & $\begin{array}{l}\text { REVENUES } \\
\text { CEIVED } \\
\end{array}$ & $\begin{array}{r}\text { TOTA } \\
\text { ALL } \\
\end{array}$ & $\begin{array}{l}\text { REVENU } \\
\text { MINERAL }\end{array}$ & $\begin{array}{l}\text { UES } \\
\text { LS }\end{array}$ & & \\
\hline $\begin{array}{r}1920-79 \\
1983\end{array}$ & $\begin{array}{r}489,461 \\
0\end{array}$ & $\$$ & $\begin{array}{r}2,066,465 \\
0\end{array}$ & $\$$ & $\begin{array}{r}89,459 \\
0\end{array}$ & $\begin{array}{r}1920-79 \\
1980 \\
1981 \\
1982 \\
1983 \\
1920-83\end{array}$ & 5 & $\begin{array}{r}596,794 \\
22,547 \\
13,549 \\
12,545 \\
15,705 \\
661,140 \\
\end{array}$ & & \\
\hline
\end{tabular}


Table 11 (cont'd):

SUMAary OF VOLDME, VAIJE, ROTALTIES AND OTtER REVENUES FROA OASHORE FEDERAL MINERAL LEASES BY STATE AND COMODITY, 1920-83

\begin{tabular}{|c|c|c|c|c|c|c|c|c|}
\hline OXIAHOKA & Volume & $\frac{N \text { BARRELS }}{\text { Market Value }}$ & & $\begin{array}{l}\text { L REVENUES } \\
\text { RECEIVED }\end{array}$ & Volume & IN & $\frac{\text { MCF }}{\text { Market Value }}$ & $\begin{array}{l}\text { GAS REVENUES } \\
\text { RECEIVED }\end{array}$ \\
\hline \multirow[t]{2}{*}{$\begin{array}{r}1920-79 \\
1980 \\
1981 \\
1982 \\
1983\end{array}$} & $\begin{array}{r}14,693,210 \\
192,874 \\
309,900 \\
387,404 \\
427,200\end{array}$ & $\begin{array}{r}45,128,492 \\
5,070,780 \\
6,067,497 \\
8,225,621 \\
12,602,400\end{array}$ & $\$$ & $\begin{array}{r}5,152,916 \\
584,136 \\
731,778 \\
995,279 \\
1,575,300\end{array}$ & $\begin{array}{r}228,938,693 \\
15,412,213 \\
12,496,628 \\
15,949,465 \\
22,611,000\end{array}$ & $\$$ & $\begin{array}{l}85,779,626 \\
26,520,082 \\
25,878,899 \\
40,117,981 \\
66,165,600\end{array}$ & $\begin{array}{r}\$ 10,601,349 \\
3,100,608 \\
3,122,229 \\
5,047,144 \\
8,270,700\end{array}$ \\
\hline & Volume & $\frac{\text { TONS }}{\text { Market Value }}$ & & $\begin{array}{l}\text { L. REVENUES } \\
\text { RECEIVED } \\
\end{array}$ & Volume & & $\frac{\text { RODUCTION }}{\text { Market Value }}$ & $\begin{array}{l}\text { OTHER REVENUES } \\
\text { RECEIVED }\end{array}$ \\
\hline $\begin{array}{r}1920-79 \\
1980 \\
1981 \\
1982 \\
1983\end{array}$ & $\begin{array}{r}8,285,042 \\
258,818 \\
257,545 \\
243,352 \\
100,559\end{array}$ & $\begin{array}{r}105,480,362 \\
11,802,057 \\
11,563,025 \\
9,453,784 \\
3,966,570\end{array}$ & $\$$ & $\begin{array}{r}2,733,128 \\
821,286 \\
1,129,504 \\
1,000,283 \\
606,141\end{array}$ & $\begin{array}{l}\text { N/A } \\
\text { N/A } \\
\text { N/A } \\
\text { N/A } \\
\text { N/A }\end{array}$ & $\gamma$ & $\begin{array}{r}32,814,813 \\
230,113 \\
181,015 \\
473,042 \\
690,600\end{array}$ & $\begin{array}{l}308,544 \\
18,990 \\
23,163 \\
59,429 \\
86,300\end{array}$ \\
\hline
\end{tabular}

TOTAL REVENUES

ALL MINERALS

\begin{tabular}{rrr}
$1920-79$ & $\$ 18,795,937$ \\
1980 & & $4,525,020$ \\
1981 & & $5,006,674$ \\
1982 & & $7,102,135$ \\
1983 & & $10,538,441$ \\
$1920-83$ & $\$ 45,968,207$ \\
\hline
\end{tabular}

\begin{tabular}{|c|c|c|c|c|c|c|c|c|}
\hline \multirow[t]{2}{*}{ CRECON } & COAL & N TONS & & COAL REVSNJES & \multirow{2}{*}{ Vol ume } & PRODUCTION & \multicolumn{2}{|c|}{ OTHER REVENUES } \\
\hline & Volume & Market & Value & RECE IVED & & Market Value & REC & CEIVED \\
\hline $\begin{array}{r}1920-79 \\
1983\end{array}$ & $\begin{array}{r}20,603 \\
0\end{array}$ & $\mathbf{\$}$ & $\begin{array}{r}374,084 \\
0\end{array}$ & $\begin{array}{r}24,147 \\
0\end{array}$ & $\begin{array}{l}\text { N/A } \\
\text { N/A }\end{array}$ & $\begin{array}{r}40 \\
0\end{array}$ & $\mathbf{\$}$ & $\begin{array}{l}5 \\
0\end{array}$ \\
\hline
\end{tabular}

TCTAL REVENUES

ALLL MINERALS

$1920-79 \$ 24,152$

1980-83 0

1920-83 \$ 24,152

\begin{tabular}{|c|c|c|c|c|c|c|c|}
\hline $\begin{array}{r}1920-79 \\
1983\end{array}$ & $\begin{array}{r}989 \\
0\end{array}$ & $\$$ & $\begin{array}{r}4,041 \\
0\end{array}$ & $\begin{array}{rr}\$ \quad 491 \\
\end{array}$ & $\begin{array}{r}2,794,991 \\
0\end{array}$ & $\begin{array}{r}952,753 \\
0\end{array}$ & $\begin{array}{r}95,385 \\
0\end{array}$ \\
\hline
\end{tabular}

TOTAL REVENUES

ALL MINERALS

\begin{tabular}{rrr}
\hline $1920-79$ & $\$$ & 95,876 \\
$1980-83$ & & 0 \\
$1920-83$ & $\$$ & 95,876 \\
\hline
\end{tabular}


Table 11 (cont'd):

SUHMARY OF VOLUHE, VALUE, ROYALTIES AND OTHER REVENUES FROM ONSHORE FEDERAL. MINERAL IFASES BY STATE AND COHODIT, 1920-83

\begin{tabular}{|c|c|c|c|c|c|c|c|c|}
\hline \multirow{2}{*}{$\begin{array}{l}\text { SOUTA } \\
\text { CAROLIRA } \\
1920-79 \\
1983\end{array}$} & \multirow{2}{*}{$\begin{array}{l}\text { OTHER } \\
\text { Volume } \\
\text { N/A } \\
\text { N/A }\end{array}$} & \multicolumn{2}{|c|}{$\frac{\text { PRODUCTION }}{\text { Market Value }}$} & \multicolumn{2}{|c|}{$\begin{array}{c}\text { OTHER REVENUES } \\
\text { RECEIVED } \\
\end{array}$} & \multicolumn{3}{|c|}{$\begin{array}{c}\text { TOTAL REVENUES } \\
\text { ALL MINERALS }\end{array}$} \\
\hline & & $\$$ & $\begin{array}{r}118,542 \\
0\end{array}$ & $\$$ & $\begin{array}{r}5,927 \\
0\end{array}$ & $\begin{array}{l}1920-79 \\
1980-83 \\
1920-83\end{array}$ & $\begin{array}{l}\$ \\
\$\end{array}$ & $\begin{array}{r}5,927 \\
0 \\
5,927 \\
\end{array}$ \\
\hline
\end{tabular}

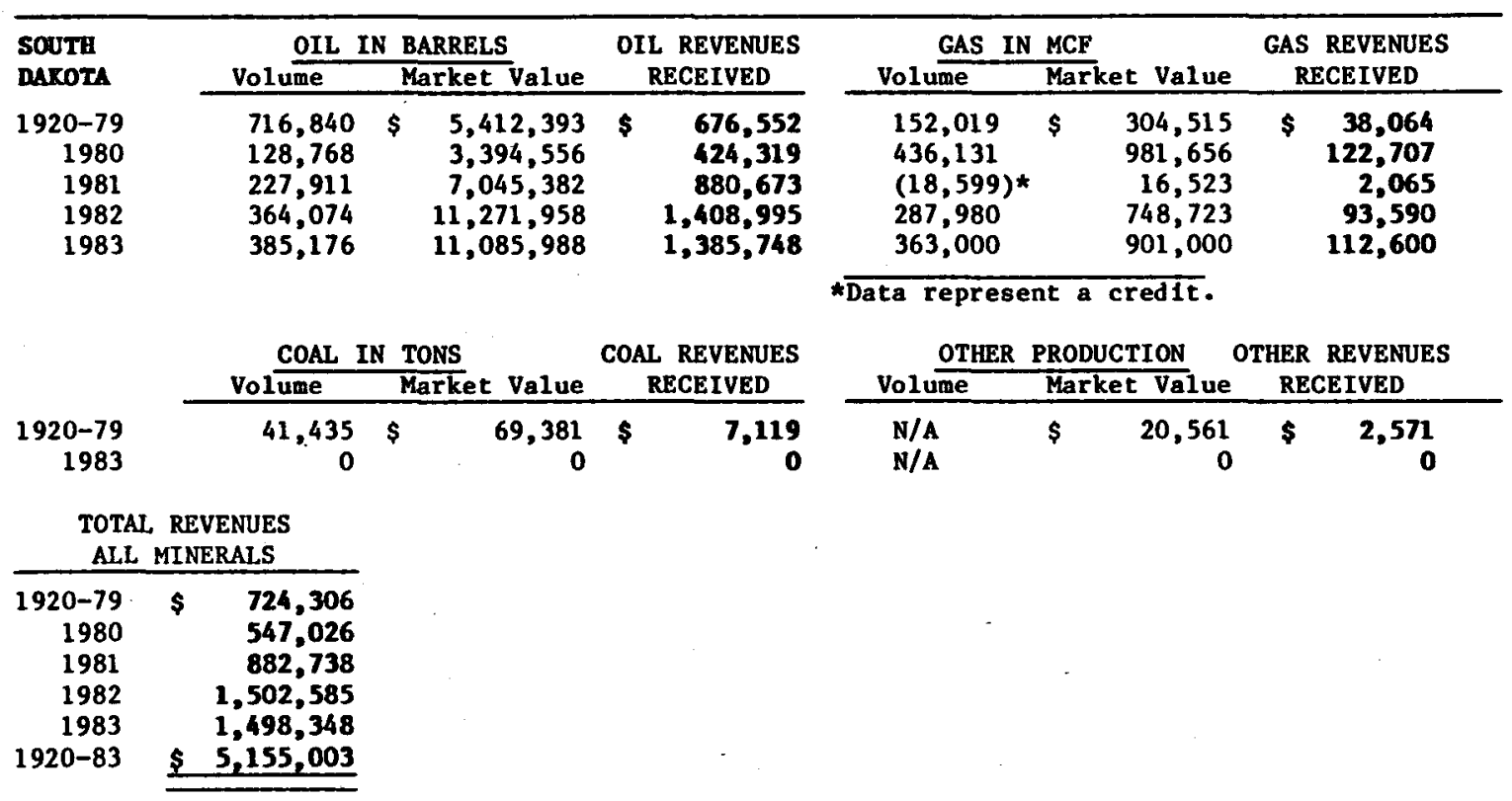

\begin{tabular}{|c|c|c|c|c|c|c|c|c|}
\hline \multirow{2}{*}{$\begin{array}{r}\text { TENNESSEE } \\
1920-79 \\
1983\end{array}$} & \multirow{2}{*}{$\begin{array}{l}\text { OTHER } \\
\text { Volume } \\
\text { N/A } \\
\text { N/A }\end{array}$} & \multicolumn{2}{|c|}{ PRODUCTION } & \multicolumn{2}{|c|}{$\begin{array}{l}\text { OTHER REVENUES } \\
\text { RECEIVED }\end{array}$} & \multicolumn{3}{|c|}{$\begin{array}{l}\text { TOTAL REVENUES } \\
\text { ALL MINERALS }\end{array}$} \\
\hline & & $\$$ & $\begin{array}{r}81,340 \\
0\end{array}$ & $\$$ & $\begin{array}{r}4,452 \\
0\end{array}$ & $\begin{array}{l}1920-79 \\
1980-83 \\
1920-83\end{array}$ & $\$$ & $\begin{array}{r}4,452 \\
0 \\
4,452 \\
\end{array}$ \\
\hline
\end{tabular}


Table 11 (cont'd):

SUMMARY OF VOLUMB, VALUE, ROTALTIES AND OTHER REVENUES FROA ONSHORE FEDERAL MINERAL LEASES BY STATE AND COHMODIT, 1920-83

\begin{tabular}{|c|c|c|c|c|c|c|c|c|c|}
\hline TExas & Volume & $\frac{\text { IN B }}{\mathrm{M}}$ & $\frac{\text { BARRELS }}{\text { larket Value }}$ & $\begin{array}{r}\text { OIL } \\
\text { R }\end{array}$ & $\begin{array}{l}\text { REVENUES } \\
\text { ECEIVED } \\
\end{array}$ & Volume & $N$ & $\frac{\text { FF }}{\text { arket Value }}$ & $\begin{array}{l}\text { GAS REVENUES } \\
\text { RECEIVED }\end{array}$ \\
\hline \multirow[t]{2}{*}{$\begin{array}{r}1920-79 \\
1980 \\
1981 \\
1982 \\
1983\end{array}$} & $\begin{array}{r}6,586,304 \\
198,682 \\
178,910 \\
146,384 \\
2,793\end{array}$ & & $\begin{array}{r}34,965,173 \\
3,255,926 \\
6,659,086 \\
4,803,470 \\
87,252\end{array}$ & & $\begin{array}{r}3,515,388 \\
373,422 \\
672,964 \\
579,969 \\
4,975\end{array}$ & $\begin{array}{r}72,950,727 \\
1,343,723 \\
1,022,162 \\
1,350,349 \\
455,470\end{array}$ & $\$$ & $\begin{array}{r}17,372,348 \\
2,261,090 \\
3,415,864 \\
4,465,045 \\
2,892,848\end{array}$ & $\begin{array}{r}\$ 2,147,239 \\
158,422 \\
185,353 \\
358,905 \\
68,353\end{array}$ \\
\hline & Vol OTHER & PRO & DUCTION & $\begin{array}{r}\text { OTHER } \\
\text { RE } \\
\end{array}$ & $\begin{array}{l}\text { REVENUES } \\
\text { CEIVED } \\
\end{array}$ & $\begin{array}{r}\text { TOTAL R } \\
\text { ALL M } \\
\end{array}$ & $\begin{array}{l}\text { EVE } \\
\text { INE }\end{array}$ & $\begin{array}{l}\text { NUES } \\
\text { RALS } \\
\end{array}$ & \\
\hline $\begin{array}{r}1920-79 \\
1980 \\
1981 \\
1982 \\
1983\end{array}$ & $\begin{array}{l}\text { N/A } \\
\text { N/A } \\
\text { N/A } \\
\text { N/A } \\
\text { N/A }\end{array}$ & $\$$ & $\begin{array}{r}9,819,897 \\
72,984 \\
83,695 \\
71,670 \\
24,000\end{array}$ & $\mathbf{s}$ & $\begin{array}{r}519,452 \\
16,719 \\
11,714 \\
9,900 \\
3,000\end{array}$ & $\begin{array}{r}1920-79 \\
1980 \\
1981 \\
1982 \\
1983 \\
1920-83\end{array}$ & $\$$ & $\begin{array}{r}6,182,079 \\
548,563 \\
870,031 \\
948,774 \\
76,328 \\
8,625,775 \\
\end{array}$ & \\
\hline
\end{tabular}

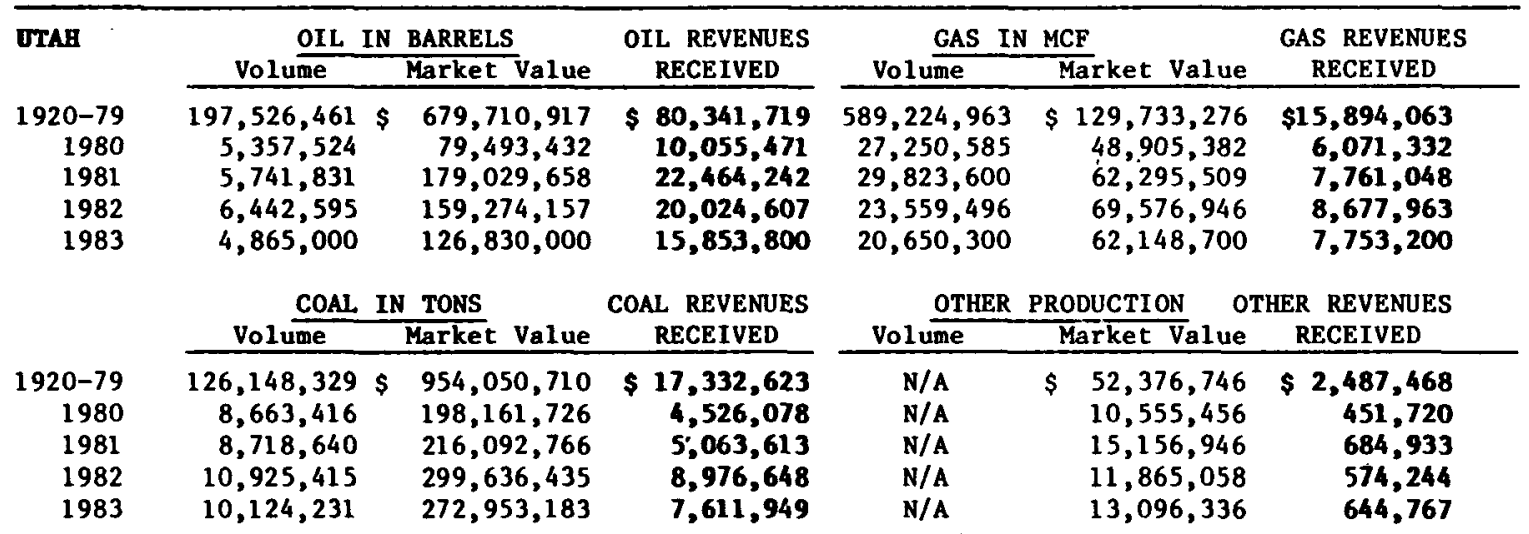

TOTAL REVENUES

ALL MINERALS

\begin{tabular}{rr}
\hline $1920-79$ & $\$ 120,449,573$ \\
1980 & $21,104,601$ \\
1981 & $35,973,836$ \\
1982 & $38,253,462$ \\
1983 & $31,863,716$ \\
$1920-83$ & $\$ 247,645,188$ \\
\hline
\end{tabular}


Table 11 (cont'd):

SUMARY OP VOLDAR, VAIUE, ROYAITTES AND OTHER REVENUES FROM ONSHORE FEDERAL MINERAL IFASES BY STATE AND COAMODIT, 1920-83

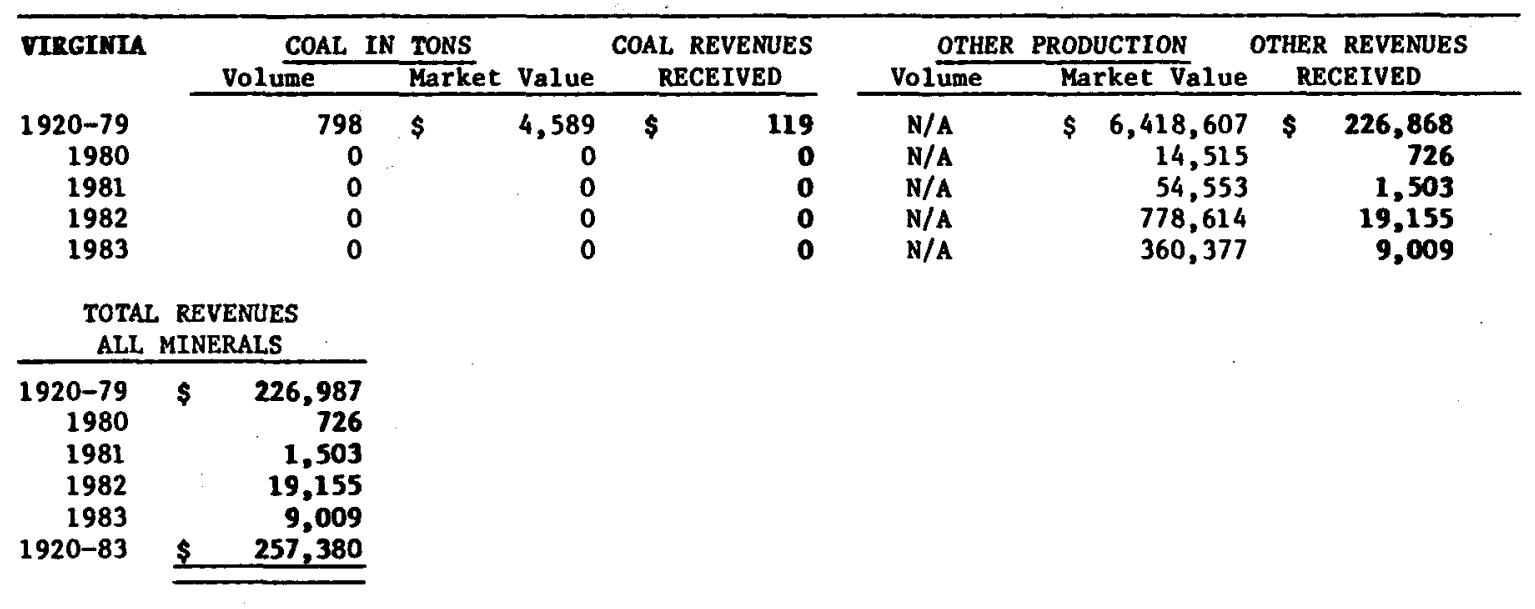

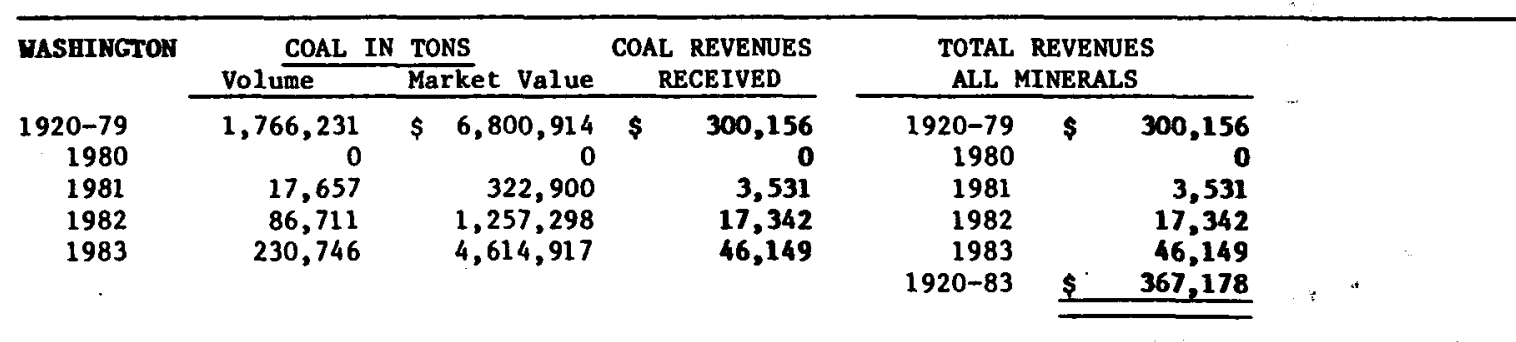

\begin{tabular}{|c|c|c|c|c|c|c|c|c|}
\hline $\begin{array}{l}\text { VEST } \\
\text { VIRGIMLA } \\
1920-79\end{array}$ & Volume & PROD & $\begin{array}{l}\text { JCTION } \\
\text { ket Value }\end{array}$ & \multicolumn{2}{|c|}{$\begin{array}{c}\text { GAS REVENUES } \\
\text { RECEIVED } \\
\end{array}$} & \multicolumn{3}{|c|}{$\begin{array}{c}\text { TOTAL REVENUES } \\
\text { ALL MINERALS }\end{array}$} \\
\hline $\begin{array}{r}1920-79 \\
1980 \\
1981 \\
1982 \\
1983\end{array}$ & $\begin{array}{r}33,397,022 \\
2,553 \\
555,729 \\
575,000 \\
520,000\end{array}$ & $\$$ & $\begin{array}{r}9,122,716 \\
2,085 \\
1,209,700 \\
1,334,000 \\
1,232,400\end{array}$ & $\$$ & $\begin{array}{r}1,523,515 \\
264 \\
151,211 \\
166,750 \\
154,000\end{array}$ & $\begin{array}{r}1920-79 \\
1980 \\
1981 \\
1982 \\
1983 \\
1920-83\end{array}$ & $\$$ & $\begin{array}{r}1,523,515 \\
264 \\
151,211 \\
166,750 \\
154,000 \\
1,995,740 \\
\end{array}$ \\
\hline
\end{tabular}




\section{4 : ONSHORE MINERAL REVENUES}

Table 11 (cont'd)

SUMMARY OF VOLUME, VALUE, ROYALTIES AND OTHER REVENUES FROM ONSHORE FEDERAL MINERAL IRASES BT STATE AND COAHODIT, 1920-83

\begin{tabular}{|c|c|c|c|c|c|c|}
\hline \multirow[t]{2}{*}{ WOAING } & \multicolumn{2}{|c|}{ OIL IN BARRELS } & \multirow{2}{*}{$\begin{array}{l}\text { OIL REVENUES } \\
\text { RECEIVED }\end{array}$} & \multicolumn{2}{|c|}{ GAS IN MCF } & \multirow{2}{*}{$\begin{array}{c}\text { GAS REVENUES } \\
\text { RECEIVED }\end{array}$} \\
\hline & Volume & Market Value & & Volume & Market Value & \\
\hline \multirow[t]{3}{*}{$\begin{array}{r}1920-79 \\
1980 \\
1981 \\
1982 \\
1983\end{array}$} & $\begin{array}{r}2,641,799,177 \\
70,575,096 \\
70,199,746 \\
67,742,499 \\
69,841,648\end{array}$ & $\begin{array}{r}\$ 9,063,283,232 \\
1,541,407,807 \\
2,259,737,039 \\
1,978,462,922 \\
1,952,074,061\end{array}$ & $\begin{array}{r}\$ 1,166,043,688 \\
191,660,745 \\
276,608,481 \\
240,821,908 \\
242,838,018\end{array}$ & $\begin{array}{r}4,182,262,524 \\
203,470,447 \\
198,187,169 \\
221,972,002 \\
232,242,658\end{array}$ & $\begin{array}{r}\$ 1,226,263,333 \\
376,737,461 \\
492,311,376 \\
670,938,161 \\
820,032,800\end{array}$ & $\begin{array}{r}\$ 145,145,743 \\
46,421,603 \\
60,877,028 \\
82,863,457 \\
101,386,800\end{array}$ \\
\hline & COAL IN & TONS & COAL & OTHER & PRODUC & THER REVENUE \\
\hline & & Market Value & & Volume & Market Value & RECEIVED \\
\hline $\begin{array}{r}920-79 \\
1980 \\
1981 \\
1982 \\
1983\end{array}$ & $\begin{array}{r}214,487,029 \\
33,433,532 \\
59,576,163 \\
63,612,335 \\
67,975,848\end{array}$ & $\begin{array}{r}\$ 1,325,117,005 \\
297,092,692 \\
580,670,187 \\
788,411,390 \\
787,230,033\end{array}$ & $\begin{array}{r}38,158,304 \\
8,700,686 \\
17,289,702 \\
21,331,355 \\
15,168,037\end{array}$ & $\begin{array}{l}\text { N/A } \\
\text { N/A } \\
\text { N/A } \\
\text { N/A } \\
\text { N/A }\end{array}$ & $\begin{array}{r}\$ 1,259,843,977 \\
163,477,094 \\
162,631,022 \\
226,432,588 \\
228,229,935\end{array}$ & $\begin{array}{r}57,192,742 \\
7,659,230 \\
8,853,391 \\
6,381,288 \\
15,876,696\end{array}$ \\
\hline
\end{tabular}

TOTAL REVENUES

ALL MINERALS

$1920-79 \$ 1,406,540,477$

$1980 \quad 254,442,264$

$1981 \quad 363,628,602$

$1982 \quad 351,398,008$

$1983 \quad 375,269,551$

$1920-83 \$ 2,751,278,902$

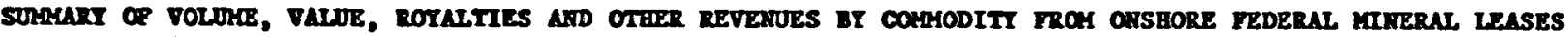
LOCATED IA 39 stATES, 1920-83

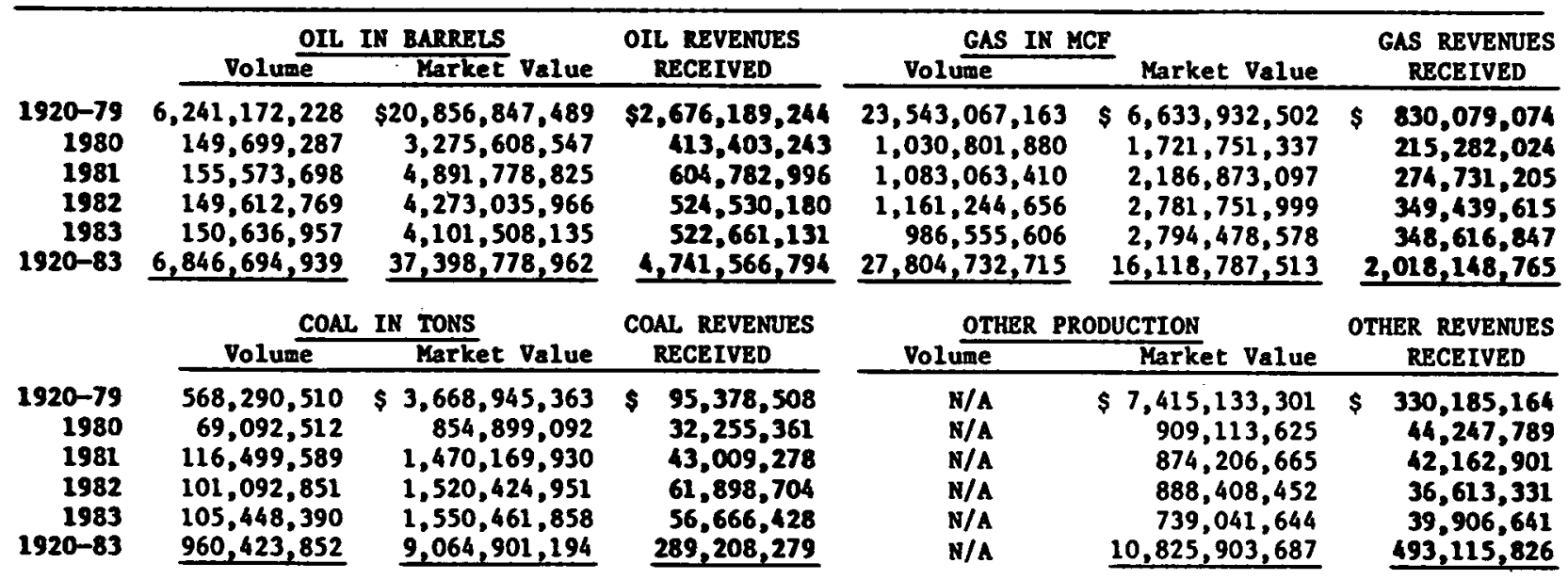

TOTAL REVENUES

ALL MINERALS

\begin{tabular}{rr}
$1920-79$ & $\$ 3,931,831,990$ \\
1980 & $705,188,417$ \\
1981 & $964,686,380$ \\
1982 & $972,481,830$ \\
1983 & $967,851,047$ \\
$1920-83$ & $\$ 7,542,039,664$ \\
\hline
\end{tabular}


Table 12:

1983 BONUSES PAID ON SALES OF ONSHORE FEDERAL MIRERAL, LEASES BY STATE AND COMMODITY

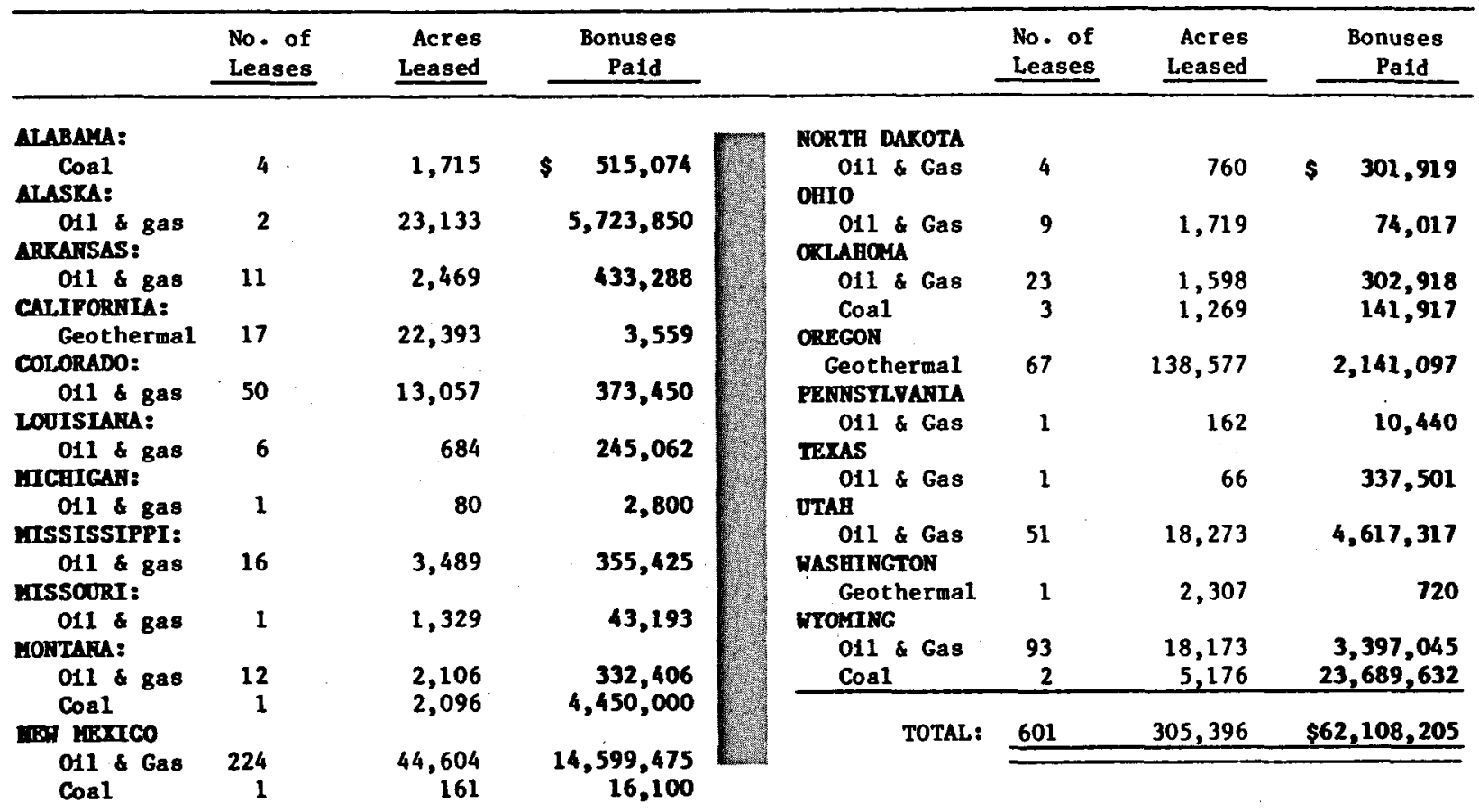

DOTB: F1scal year data in Public Land Stat1st1cs, 1983, Bureau of Land Management. 
36 : ONSHORE MINERAL REVENUES

Table 13:

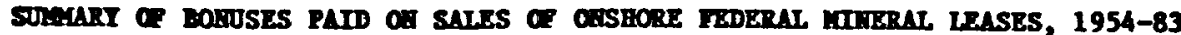

\begin{tabular}{|c|c|c|c|c|c|c|}
\hline & \multicolumn{2}{|c|}{ OIL \& GAS } & \multicolumn{2}{|c|}{ OTBER MINERALS } & \multicolumn{2}{|c|}{ TOTALS } \\
\hline & $\begin{array}{c}\text { Acres } \\
\text { Leased }\end{array}$ & $\begin{array}{c}\text { Bonuses } \\
\text { Paid } \\
\end{array}$ & $\begin{array}{r}\text { Acres } \\
\text { Leased } \\
\end{array}$ & $\begin{array}{c}\text { Bonuses } \\
\text { Pald } \\
\end{array}$ & $\begin{array}{r}\text { Acres } \\
\text { Leased }\end{array}$ & $\begin{array}{c}\text { Bonuses } \\
\text { Pald } \\
\end{array}$ \\
\hline $\begin{array}{l}1954 \\
1955 \\
1956 \\
1957 \\
1958 \\
1959 \\
1960 \\
1961 \\
1962 \\
1963 \\
1964 \\
1965 \\
1966 \\
1967 \\
1968 \\
1969 \\
1970 \\
1971 \\
1972 \\
1973 \\
1974 \\
1975 \\
1976 \\
1977 \\
1978 \\
1979 \\
1980 \\
1981 \\
1982 \\
1983\end{array}$ & $\begin{array}{r}7,726 \\
16,145 \\
21,497 \\
182,233 \\
10,564 \\
32,283 \\
21,947 \\
27,466 \\
32,711 \\
53,124 \\
20,335 \\
46,572 \\
35,453 \\
61,665 \\
71,505 \\
36,969 \\
38,724 \\
52,102 \\
88,326 \\
89,315 \\
65,247 \\
112,401 \\
74,616 \\
80,529 \\
76,438 \\
62,447 \\
71,623 \\
126,070 \\
991,186 \\
131,702\end{array}$ & $\begin{array}{r}255,912 \\
833,305 \\
1,241,479 \\
1,197,405 \\
274,432 \\
882,831 \\
668,865 \\
12,395,374 \\
7,059,451 \\
2,089,236 \\
649,918 \\
1,805,432 \\
572,837 \\
764,296 \\
972,523 \\
754,543 \\
478,890 \\
1,163,136 \\
1,117,543 \\
2,203,370 \\
2,295,623 \\
8,202,991 \\
4,934,208 \\
7,820,947 \\
12,705,965 \\
7,597,171 \\
22,048,947 \\
103,314,389 \\
95,304,216 \\
31,150,106\end{array}$ & $\begin{array}{r}4,362 \\
3,319 \\
7,608 \\
14,663 \\
28,968 \\
20,474 \\
11,748 \\
25,863 \\
41,507 \\
54,244 \\
32,290 \\
30,009 \\
48,979 \\
47,606 \\
95,303 \\
18,493 \\
28,706 \\
-133 \\
24,493 \\
362 \\
--2 \\
-574 \\
6,475 \\
9,897 \\
60,034 \\
352,510 \\
173,694\end{array}$ & $\begin{array}{r}59,260 \\
414,804 \\
170,293 \\
297,265 \\
1,927,505 \\
305,847 \\
111,291 \\
1,030,725 \\
223,379 \\
475,288 \\
1,112,824 \\
1,072,714 \\
1,071,869 \\
936,656 \\
3,102,240 \\
370, \overline{395} \\
7,627,114 \\
33, \overline{635} \\
49,192,426 \\
3,618 \\
30,958,099\end{array}$ & $\begin{array}{r}12,088 \\
19,464 \\
29,105 \\
196,896 \\
39,532 \\
52,757 \\
33,695 \\
53,329 \\
74,218 \\
107,368 \\
52,625 \\
76,581 \\
84,432 \\
109,271 \\
166,808 \\
36,969 \\
57,217 \\
80,808 \\
88,326 \\
89,448 \\
89,740 \\
112,763 \\
74,616 \\
80,529 \\
77,012 \\
68,922 \\
81,520 \\
186,104 \\
143,696 \\
305,396\end{array}$ & $\begin{array}{r}315,172 \\
1,248,109 \\
1,411,772 \\
1,494,670 \\
2,201,937 \\
1,188,678 \\
780,156 \\
13,426,099 \\
7,282,830 \\
2,564,524 \\
1,762,742 \\
2,878,146 \\
1,644,706 \\
1,700,952 \\
4,074,763 \\
754,543 \\
849,285 \\
8,790,250 \\
1,117,543 \\
2,237,005 \\
451,488,049 \\
8,206,609 \\
4,934,208 \\
7,820,947 \\
12,737,345 \\
8,413,179 \\
22,982,316 \\
128,673,005 \\
125,270,858 \\
62,108,205\end{array}$ \\
\hline TOTAL: & $2,738,921$ & $\$ 3332,755,341$ & $1,142,314$ & $\$ 557,603,262$ & $3,881,235$ & $\$ 890,358,603$ \\
\hline
\end{tabular}

SOURCE: Public Land Statistics, 1983, Bureau of Land Management.

Open pit copper mine (upper right) in the San Xavier $\emptyset$ Reservation of the Papago Indian Tribe in Arizona. Ore concentrates are processed at the nearby Asarco Mission Mill. 


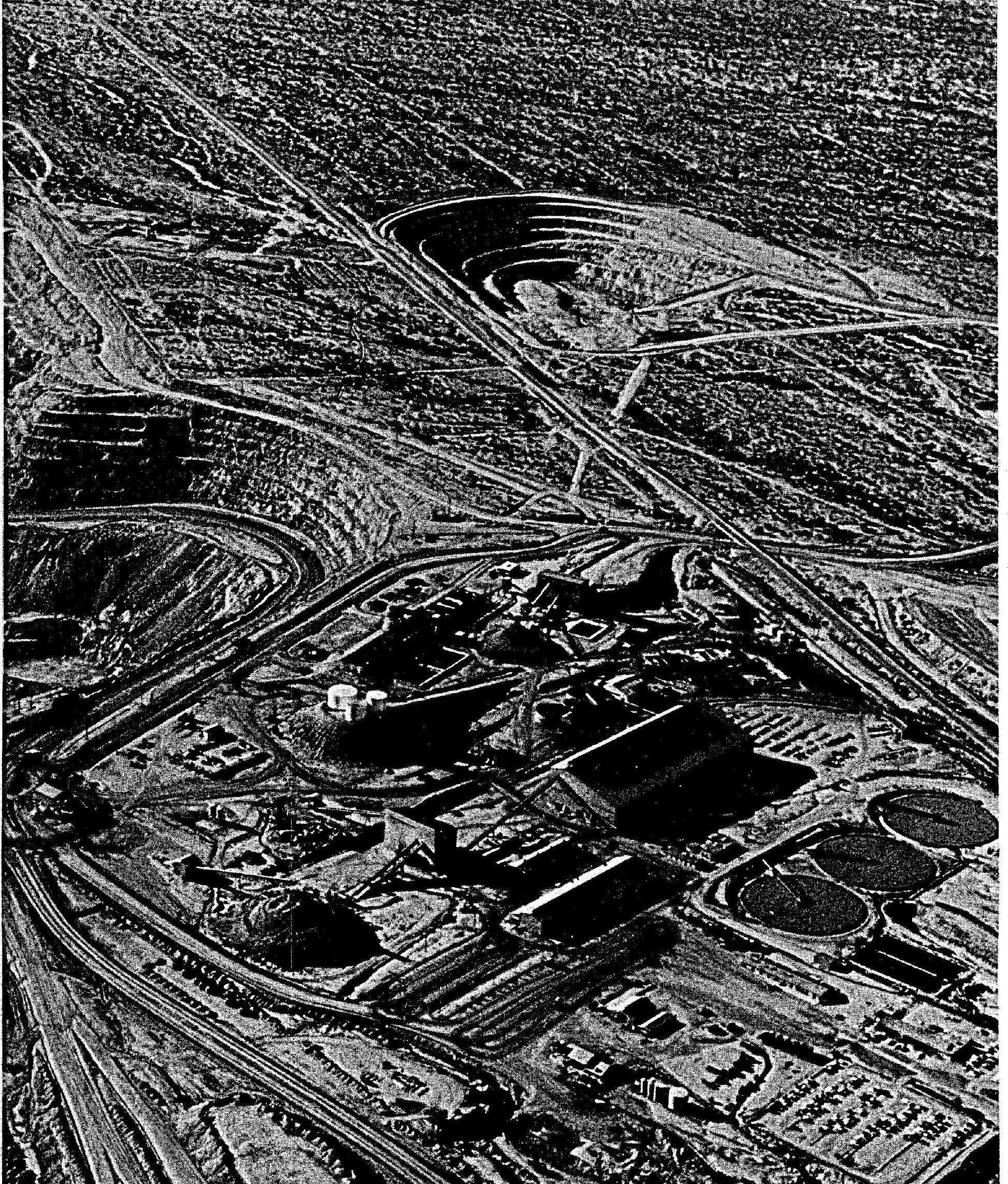




\section{INDIAN MINERAL REVENUES}

The market value of all fluid and solid minerals extracted from Indian leases amounted to $\$ 6.8$ billion from 1937 to 1979 . For the same 42 years, Indian royalty revenues totalled $\$ 809$ million. Since 1979 , the market value of oil, gas, coal, and other minerals production from Indian lands amounted to $\$ 5.3$ billion, and the royalties totalled $\$ 599$ million.

In 1983, all Indian royalties (except Osage) totalled $\$ 156,127,450$ for an overall decline of 4.1 percent (Table 14, page 46). Natural gas production led the downward trend with a sharp 11.5 percent drop--from 133 to 118 billion cubic feet. Nevertheless, gas royalties only declined 6.2 percent because the market value of gas rose from $\$ 2.82$ to $\$ 3.02$ per Mcf.

Oil production rose 1.5 percent on Indian leases-approximately twice the gains for onshore Federal oil. Nevertheless, royal ty revenues followed the market and were down, from $\$ 92,559,824$ in 1982 to $\$ 90,320,416$ in 1983.

As of December 31, 1983, there were 87 more producing and producible oil and gas leases, licenses, and permits under supervision on Indian lands than in 1982-a total of 3,865 (Table 19). Coal leasing activity for the same period was unchanged (Table 20). But the total number of producing leases of other minerals was up slightly-from 33 to 36 (Table 21).

Floure t:

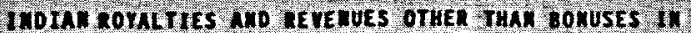

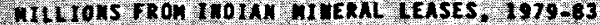

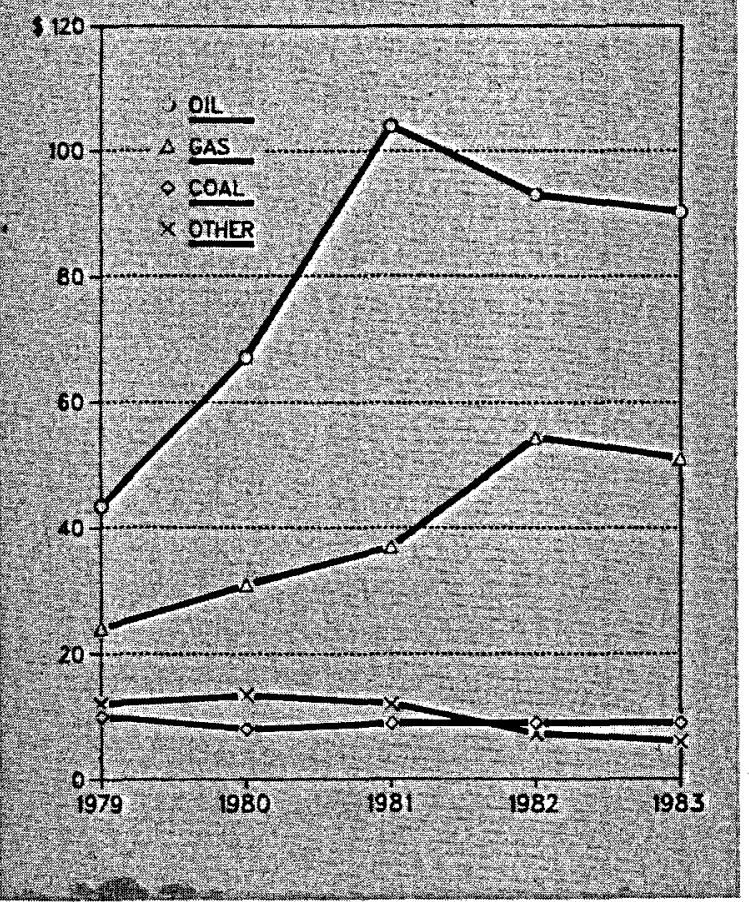

Coal royalties were also up-from $\$ 8,527,548$ to $\$ 8,762,711$ or 2.8 percent--although production declined slightly. In 1983, 28.4 million tons of coal were extracted from Indian lands as contrasted with 28.9 million tons the previous year. As before, most of this coal, valued at $\$ 478$ million, was produced from Navajo tribal lands in Arizona and New Mexico.

During 1983, several energy-producing Indian Tribes began to test the provisions of the Indian Mineral Development Act of 1982. Thus, a number of joint ventures and participation agreements were being developed or negotiated by year's end. 


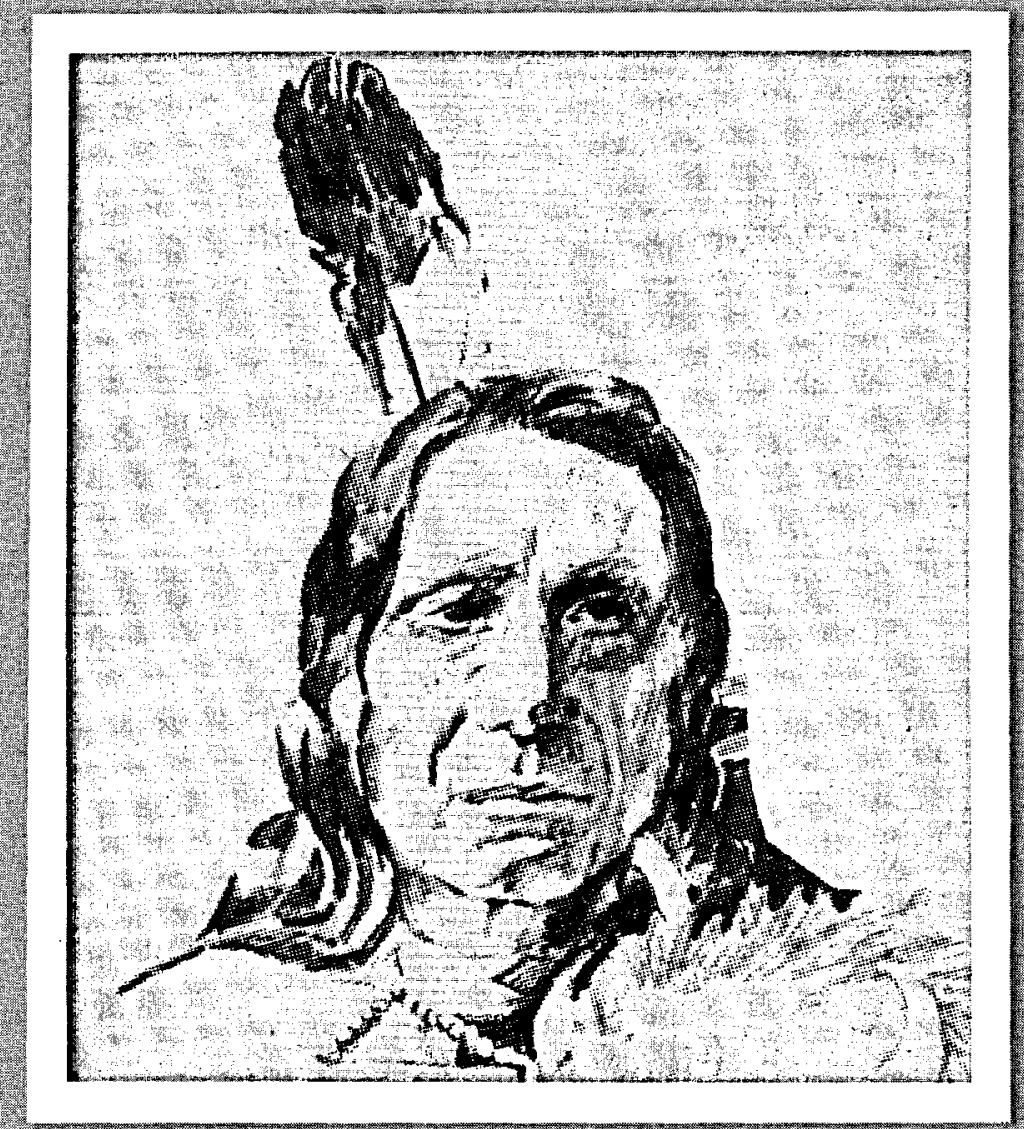

INDIAN IRIBES, NATIONS, AND COUNCILS WTTH VINERAL IRASES PRODUCING IN 1983

\begin{tabular}{|c|c|}
\hline $\begin{array}{l}\text { Absentee } \\
\text { Apache } \\
\text { Arapahoe } \\
\text { Assinibolne } \\
\text { Blackfeet } \\
\text { Caddo } \\
\text { Cherokee } \\
\text { Cherokee-Choctaw-Chickasaw } \\
\text { Cheyenne } \\
\text { Cheyenne River Sloux } \\
\text { Chickasaw } \\
\text { Chickasaw-Choctaw } \\
\text { Chippewa-Cree } \\
\text { Choctaw } \\
\text { Cltizens Band of Potawatomt } \\
\text { Clackamas } \\
\text { Comanche } \\
\text { Creek } \\
\text { Crow }\end{array}$ & $\begin{array}{l}\text { Iowa } \\
\text { Jicarlila Apache } \\
\text { Klckapoo } \\
\text { Kiowa } \\
\text { laguna Pueblo } \\
\text { Navajo } \\
\text { Osage } \\
\text { Otoe } \\
\text { Palute } \\
\text { Pala Band-Mission } \\
\text { Papago } \\
\text { Pawnee } \\
\text { Pima } \\
\text { Ponca } \\
\text { Potawatomi } \\
\text { Ouapaw } \\
\text { Sac \& Fox } \\
\text { Saglnaw ChIppewa } \\
\text { Sandia Pueblo }\end{array}$ \\
\hline
\end{tabular}

San Felipe Fueblo

San Ildefonso Pueblo

Seminole

Shawnee

Shoshone Arapahoe

Southern Ute

Spokane

Taos Pueblo

Three Affllitated Tribes: Artckara Gros Ventre Mand an

Turtle Mountaln Band of chippewa.

Turtle Mountain Chi ppewa Uintah \& Ouray

Ute Mountain

wichita

Takima

Yavapa1 
Table 14:

SUROARY OF VOLUR, VAIJE, ROTALTIES AND OTHER REVENUES FROA INDLAN GINERAL LEASES BT STATE AND COHAODIT, 1937-83

\begin{tabular}{|c|c|c|c|c|c|c|c|c|}
\hline \multirow{2}{*}{$\begin{array}{r}\text { AIASKA } \\
1937-79 \\
1983\end{array}$} & \multicolumn{2}{|c|}{$\begin{array}{cc}\text { GAS IN MCF } \\
\text { Volume } & \text { Market } \\
\end{array}$} & \multicolumn{3}{|c|}{$\begin{array}{c}\text { GAS REVENUES } \\
\text { RECE IVED } \\
\end{array}$} & \multicolumn{3}{|c|}{$\begin{array}{c}\text { TOTAL REVENUES } \\
\text { ALL MINERALS }\end{array}$} \\
\hline & $\begin{array}{r}277,556 \\
0\end{array}$ & $\$$ & $\begin{array}{r}39,269 \\
0\end{array}$ & $\$$ & $\begin{array}{r}6,541 \\
0\end{array}$ & $\begin{array}{l}1937-79 \\
1980-83 \\
1937-83\end{array}$ & $\$$ & $\begin{array}{r}6,541 \\
0 \\
6,541 \\
\end{array}$ \\
\hline
\end{tabular}

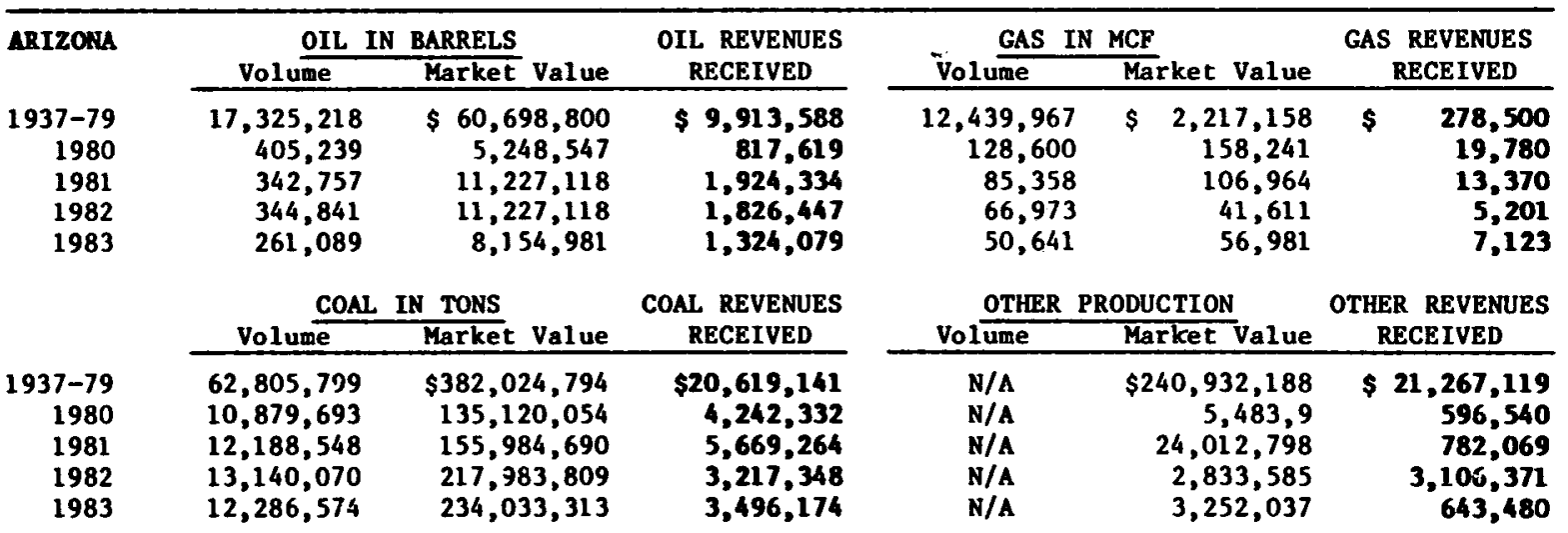

TOTAL REVENUES

ALL MINERALS

\begin{tabular}{rr}
$1937-79$ & $\$ 52,078,708$ \\
1980 & $5,676,271$ \\
1981 & $8,389,037$ \\
1982 & $8,155,367$ \\
1983 & $5,470,856$ \\
$1937-83$ & $\$ \quad 79,770,239$ \\
\hline
\end{tabular}

\begin{tabular}{|c|c|c|c|c|c|c|c|c|}
\hline CALIFORNIA & Volume & PRO & $\begin{array}{l}\text { DUCTION } \\
\text { Market Value }\end{array}$ & OTHE & $\begin{array}{l}\text { R REVENUES } \\
\text { RECEIVED } \\
\end{array}$ & $\begin{array}{l}\text { TOTA } \\
\text { ALI }\end{array}$ & $\mathbf{L}$ & $\begin{array}{l}\text { VENUES } \\
\text { ERALS }\end{array}$ \\
\hline $\begin{array}{c}1937-79 \\
1980 \\
1981 \\
1982 \\
1983\end{array}$ & $\begin{array}{l}\text { N/A } \\
\text { N/A } \\
\text { N/A } \\
\text { N/A } \\
\text { N/A }\end{array}$ & $\$$ & $\begin{array}{r}15,800,391 \\
508,136 \\
274,393 \\
199,867 \\
200,574\end{array}$ & $\$$ & $\begin{array}{r}1,153,304 \\
146,573 \\
88,080 \\
131,207 \\
72,304\end{array}$ & $\begin{array}{r}1937-79 \\
1980 \\
1981 \\
1982 \\
1983 \\
1937-83\end{array}$ & $\$$ & $\begin{array}{r}1,153,304 \\
146,573 \\
88,080 \\
131,207 \\
72,304 \\
1,591,468\end{array}$ \\
\hline
\end{tabular}

rort: Calendar year data Include ofl volume in barrels (42 U.S. gallons), gas In thousand cubic feet (Mcf), coal in short tons $(\$ 2,000)$, revenues in dollars. "Other" Includes certain of the solid and fluid minerals footnoted in Table 2. 
Table 14 (cont'd):

SUMMARY OF VOLUKE, VAUUE, ROYALTIES AND OTHER REVENUES FROM THDIAN MIMBRAL LEASES BY STATE AND COMMODITI, 1937-83,

\begin{tabular}{|c|c|c|c|c|c|c|c|c|c|c|}
\hline \multirow{3}{*}{$\begin{array}{c}\text { COLORADO } \\
1937-79 \\
1980 \\
1981 \\
1982 \\
1983\end{array}$} & \multirow{2}{*}{\multicolumn{2}{|c|}{ Volume $\frac{\text { OIL IN BARRELS }}{\text { Market }}$}} & \multirow{2}{*}{ Value } & \multirow{2}{*}{\multicolumn{2}{|c|}{$\begin{array}{c}\text { OIL REVENUES } \\
\text { RECEIVED } \\
\end{array}$}} & \multicolumn{3}{|c|}{ GAS IN MCF } & \multicolumn{2}{|c|}{ GAS REVENUES } \\
\hline & & & & & & Volume & \multicolumn{2}{|c|}{ Market Value } & \multicolumn{2}{|c|}{ RECEIVED } \\
\hline & $\begin{array}{r}2,785,672 \\
54,140 \\
66,191 \\
51,197 \\
51,569\end{array}$ & $\begin{array}{l}8,7 \\
1,0 \\
1,9 \\
1,6 \\
1,3\end{array}$ & $\begin{array}{l}, 721,043 \\
, 043,361 \\
, 947,320 \\
, 637,484 \\
, 375,080\end{array}$ & $\mathbf{s}$ & $\begin{array}{r}1,266,115 \\
153,401 \\
275,459 \\
231,967 \\
200,780\end{array}$ & $\begin{array}{r}418,815,699 \\
15,716,835 \\
16,622,556 \\
17,926,860 \\
15,775,700\end{array}$ & \multicolumn{2}{|c|}{$\begin{array}{r}93,944,748 \\
20,421,385 \\
29,718,372 \\
36,794,125 \\
32,971,213\end{array}$} & \multicolumn{2}{|c|}{$\begin{array}{r}\$ 11,832,566 \\
2,582,202 \\
3,783,832 \\
4,672,661 \\
4,121,402\end{array}$} \\
\hline & \multicolumn{2}{|c|}{ COAL IN TONS } & & \multicolumn{2}{|c|}{ COAL REVENUES } & \multicolumn{3}{|c|}{ OTHER PRODUCTION } & \multirow{2}{*}{\multicolumn{2}{|c|}{$\begin{array}{l}\text { OTHER REVENUES } \\
\text { RECEIVED }\end{array}$}} \\
\hline & Volume & Market & Value & & & Volume & & et Value & & \\
\hline $\begin{array}{r}1937-79 \\
1980 \\
1981 \\
1982 \\
1983\end{array}$ & $\begin{array}{r}1,659 \\
0 \\
0 \\
0 \\
0\end{array}$ & $\$$ & $\begin{array}{r}7,723 \\
0 \\
0 \\
0 \\
0\end{array}$ & $\$$ & $\begin{array}{r}198 \\
0 \\
0 \\
0 \\
0\end{array}$ & $\begin{array}{l}\text { N/A } \\
\text { N/A } \\
\text { N/A } \\
\text { N/A } \\
\text { N/A }\end{array}$ & \$ & $\begin{array}{r}583,578 \\
150,031 \\
109,432 \\
45,602 \\
83,800\end{array}$ & $\$$ & $\begin{array}{r}66,659 \\
8,463 \\
34,981 \\
2,533 \\
4,700\end{array}$ \\
\hline
\end{tabular}

TOTAL REVENUES

ALL MINERALS

\begin{tabular}{rrr}
\hline $1937-79$ & $\$ 13,165,538$ \\
1980 & & $2,743,884$ \\
1981 & & $4,094,272$ \\
1982 & & $4,907,161$ \\
1983 & $4,326,882$ \\
$1937-83$ & $\$ 29,237,737$ \\
\hline
\end{tabular}

\begin{tabular}{|c|c|c|c|c|c|c|c|}
\hline FLORIDA & Volume & $\frac{\text { BARRELS }}{\text { Market }}$ & Value & $\begin{array}{l}\text { OIL REVENUES } \\
\text { RECEIVED }\end{array}$ & Volume & $\frac{\text { PRODUCTION }}{\text { Market Value }}$ & $\begin{array}{c}\text { OTHER REVENUES } \\
\text { RECEIVED }\end{array}$ \\
\hline $\begin{array}{r}1937-79 \\
1983\end{array}$ & $\begin{array}{r}4,688 \\
0\end{array}$ & $\$$ & $\begin{array}{r}51,367 \\
0\end{array}$ & $\begin{array}{r}6,422 \\
0\end{array}$ & $\begin{array}{l}\text { N/A } \\
\text { N/A }\end{array}$ & $\begin{array}{r}451,024 \\
0\end{array}$ & $\begin{array}{r}45,103 \\
0\end{array}$ \\
\hline
\end{tabular}

TOTAL REVENUES

ALL MINERALS

\begin{tabular}{llr}
\hline $1937-79$ & $\$$ & 51,525 \\
$1980-83$ & & 0 \\
$1937-83$ & $\$$ & 51,525 \\
\hline
\end{tabular}

\begin{tabular}{|c|c|c|c|c|c|c|c|}
\hline IDAHO & Vollume & & $\begin{array}{l}\text { DuCTION } \\
\text { Market value }\end{array}$ & $\begin{array}{c}\text { OTHER REVENUES } \\
\text { RECEIVED } \\
\end{array}$ & $\begin{array}{r}\text { TOT } \\
\text { AL }\end{array}$ & $\begin{array}{l}\mathrm{AL} \\
\mathrm{L} M\end{array}$ & $\begin{array}{l}\text { REVENUES } \\
\text { INERALS }\end{array}$ \\
\hline $\begin{array}{r}1937-79 \\
1980 \\
1981 \\
1982 \\
1983\end{array}$ & $\begin{array}{l}N / A \\
N / A \\
N / A \\
N / A \\
N / A\end{array}$ & $\$$ & $\begin{array}{r}140,159,852 \\
14,548,959 \\
17,853,762 \\
0 \\
0\end{array}$ & $\begin{array}{r}11,627,593 \\
1,454,896 \\
1,785,376 \\
0 \\
0\end{array}$ & $\begin{array}{r}1937-79 \\
1980 \\
1981 \\
1982 \\
1983 \\
1937-83\end{array}$ & $\mathbf{\$}$ & $\begin{array}{r}11,627,593 \\
1,454,896 \\
1,785,376 \\
0 \\
0 \\
14,867,865 \\
\end{array}$ \\
\hline
\end{tabular}


Table 14 (cont'd):

SUMAARY OR VOLUNE, VALUE, ROTALTIES AKD OTHER REVENUES FROA INDIAN MINERAL LEASES BT STATE AND COMHODITY, 1937-83

\begin{tabular}{|c|c|c|c|c|c|c|c|c|}
\hline $\begin{array}{l}\text { MICHIGAN } \\
\text { 1937-79 }\end{array}$ & $\frac{\frac{\text { OIL IN }}{\text { Volume }}}{29,535}$ & $\frac{\text { BARRELS }}{\text { Market }}$ & Value & \multicolumn{2}{|c|}{$\begin{array}{c}\text { OIL REVENUES } \\
\text { RECEIVED } \\
\end{array}$} & \multicolumn{3}{|c|}{$\begin{array}{c}\text { TOTAL REVENUES } \\
\text { ALL MINERALS }\end{array}$} \\
\hline $\begin{array}{c}1937-79 \\
1980 \\
1981 \\
1982 \\
1983\end{array}$ & $\begin{array}{r}29,535 \\
82 \\
326 \\
285 \\
245\end{array}$ & 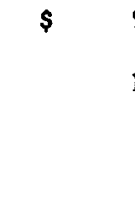 & $\begin{array}{r}95,449 \\
3,040 \\
12,303 \\
8,550 \\
7,150\end{array}$ & $\$$ & $\begin{array}{r}14,859 \\
247 \\
1,553 \\
1,070 \\
894\end{array}$ & $\begin{array}{r}1937-79 \\
1980 \\
1981 \\
1982 \\
1983 \\
1937-83\end{array}$ & $\$$ & $\begin{array}{r}14,859 \\
247 \\
1,553 \\
1,070 \\
894 \\
18,623 \\
\end{array}$ \\
\hline
\end{tabular}

\begin{tabular}{|c|c|c|c|c|c|c|c|c|}
\hline \multirow{2}{*}{$\begin{array}{c}\text { MTRNESOTA } \\
1937-79 \\
1983\end{array}$} & \multirow{2}{*}{$\begin{array}{l}\text { OTHER } \\
\text { Volume } \\
\text { N/A } \\
\text { N/A }\end{array}$} & \multicolumn{2}{|c|}{$\frac{\text { PRODUCTION }}{\text { Market Value }}$} & \multicolumn{2}{|c|}{$\begin{array}{c}\text { OTHER REVENUES } \\
\text { RECEIVED }\end{array}$} & \multicolumn{3}{|c|}{$\begin{array}{c}\text { TOTAL REVENUES } \\
\text { ALL MINERALS }\end{array}$} \\
\hline & & $\$$ & $\begin{array}{r}178,461 \\
0\end{array}$ & $\$$ & $\begin{array}{r}6,455 \\
0\end{array}$ & $\begin{array}{l}1937-79 \\
1980-83 \\
1937-83\end{array}$ & $\$$ & $\begin{array}{r}6,455 \\
0 \\
6,455 \\
\end{array}$ \\
\hline
\end{tabular}

\begin{tabular}{|c|c|c|c|c|c|c|c|}
\hline \multirow{2}{*}{$\begin{array}{r}\text { MONTARA } \\
1937-79 \\
1980 \\
1981 \\
1982 \\
1983\end{array}$} & \multicolumn{2}{|c|}{ Volume $\frac{\text { OIL IN BARRELS }}{\text { Market }}$ Value } & $\begin{array}{l}\text { OIL REVENUES } \\
\text { RECEIVED }\end{array}$ & \multicolumn{2}{|c|}{ GAS IN MCF } & \multicolumn{2}{|c|}{$\begin{array}{c}\text { GAS REVENUES } \\
\text { RECEIVED }\end{array}$} \\
\hline & $\begin{array}{r}80,151,450 \\
1,303,624 \\
1,114,025 \\
1,303,246 \\
1,327,100\end{array}$ & $\begin{array}{r}\$ 265,469,405 \\
39,911,288 \\
39,614,722 \\
40,515,438 \\
39,963,050\end{array}$ & $\begin{array}{r}\$ 36,054,232 \\
6,038,578 \\
4,694,940 \\
5,837,203 \\
5,598,050\end{array}$ & $\begin{array}{r}16,203,146 \\
1,037,248 \\
1,258,066 \\
1,381,849 \\
1,210,000\end{array}$ & $\begin{array}{r}5,021,428 \\
1,696,670 \\
2,262,655 \\
2,824,263 \\
2,856,000\end{array}$ & $\$$ & $\begin{array}{l}764,613 \\
269,830 \\
340,476 \\
447,645 \\
450,300\end{array}$ \\
\hline & Volume & $\frac{\text { IN TONS }}{\text { Market Value }}$ & $\begin{array}{c}\text { COAL REVENUES } \\
\text { RECEIVED }\end{array}$ & Volume & $\frac{\text { PRODUCTION }}{\text { Market Value }}$ & $\begin{array}{r}\text { OTHER } \\
\text { REC } \\
\end{array}$ & $\begin{array}{l}\text { REVENUES } \\
\text { EIVED }\end{array}$ \\
\hline $\begin{array}{r}1937-79 \\
1980 \\
1981 \\
1982 \\
1983\end{array}$ & $\begin{array}{r}23,151,017 \\
2,742,031 \\
3,074,386 \\
3,704,018 \\
1,729,209\end{array}$ & $\begin{array}{r}\$ 146,231,960 \\
23,042,720 \\
21,025,506 \\
41,369,320 \\
35,591,448\end{array}$ & $\begin{array}{l}9,740,790 \\
1,609,606 \\
1,424,677 \\
2,603,346 \\
1,805,955\end{array}$ & $\begin{array}{l}\text { N/A } \\
\text { N/A } \\
\text { N/A } \\
\text { N/A } \\
\text { N/A }\end{array}$ & $\begin{array}{r}\$ 15,431,806 \\
196,899 \\
550,454 \\
351,982 \\
486,000\end{array}$ & $\$$ & $\begin{array}{r}861,367 \\
9,302 \\
60,769 \\
19,062 \\
26,000\end{array}$ \\
\hline
\end{tabular}

TOTAL REVENUES

\begin{tabular}{rr}
\multicolumn{2}{c}{ ALL } \\
\hline $1937-79$ & $\$ 47,421,002$ \\
$: 980$ & $7,927,316$ \\
1981 & $6,520,862$ \\
1982 & $8,907,256$ \\
1983 & $7,880,305$ \\
$1937-83$ & $\$ 78,656,741$ \\
\hline
\end{tabular}

\begin{tabular}{|c|c|c|c|c|c|c|}
\hline $\begin{array}{r}\text { IEVADA } \\
1937-79 \\
1980\end{array}$ & $\frac{\text { Volume }}{\text { N/A }}$ & PRODUCTION & $\begin{array}{c}\text { OTHER REVENUES } \\
\text { RECEIVED } \\
\end{array}$ & \multicolumn{3}{|c|}{$\begin{array}{c}\text { TOTAL REVENUES } \\
\text { ALL MINERALS } \\
\end{array}$} \\
\hline $\begin{array}{r}1937-79 \\
1980 \\
1981 \\
1982 \\
1983\end{array}$ & $\begin{array}{l}\text { N/A } \\
\text { N/A } \\
\text { N/A } \\
\text { N/A } \\
\text { N/A }\end{array}$ & $\begin{array}{r}1,450,203 \\
274,331 \\
1,167,237 \\
362,850 \\
83,204\end{array}$ & $\begin{array}{r}194,870 \\
54,649 \\
40,802 \\
28,360 \\
8,957\end{array}$ & $\begin{array}{r}1937-79 \\
1980 \\
1981 \\
1982 \\
1983 \\
1937-83\end{array}$ & $\$$ & $\begin{array}{r}194,870 \\
54,649 \\
40,802 \\
28,360 \\
8,957 \\
327,638\end{array}$ \\
\hline
\end{tabular}


Table 14 (cont'd):

SUMMARY OF VOLUME, VALUE, ROYALTIES AND OTHER REVERURS FROH INDIAN MLNERAL LEASES BY STATE AND COMMODITY, 1937-83

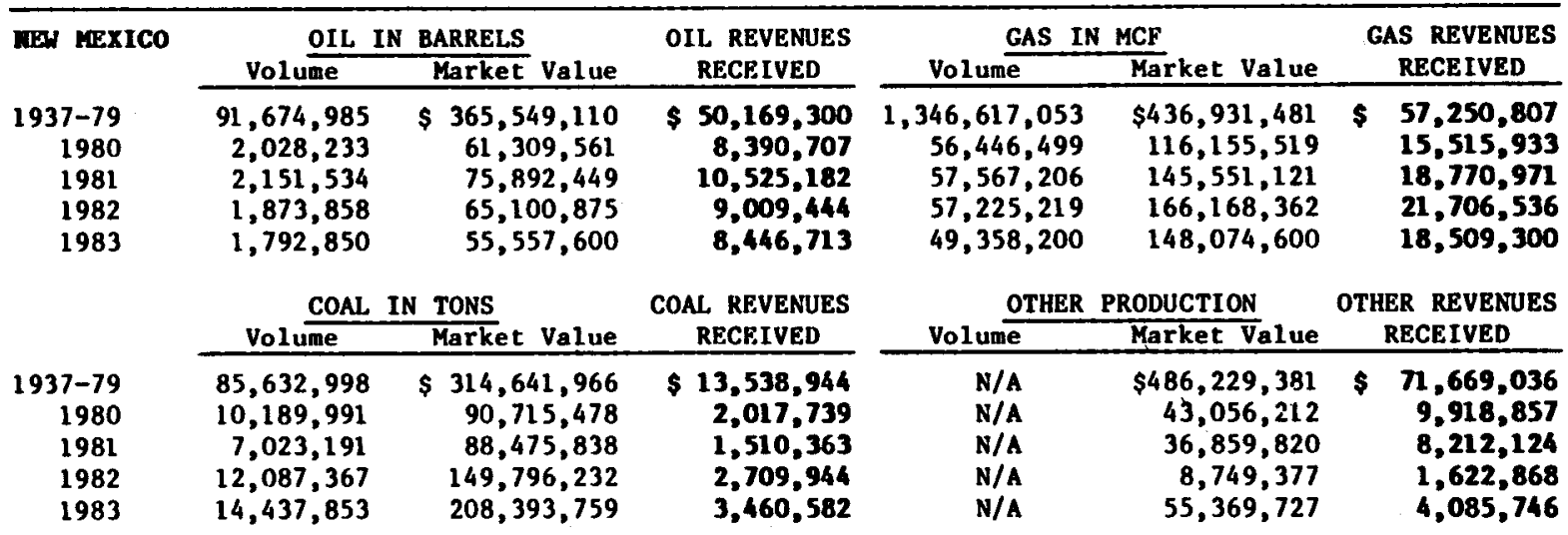

TOTAL REVENUES

ALL. MINERALS

\begin{tabular}{rr}
\hline $1937-79$ & $\$ 192,628,087$ \\
1980 & $35,843,236$ \\
1981 & $39,018,640$ \\
1982 & $35,048,792$ \\
1983 & $34,502,341$ \\
$1937-83$ & $\$ 337,041,096$ \\
\hline
\end{tabular}

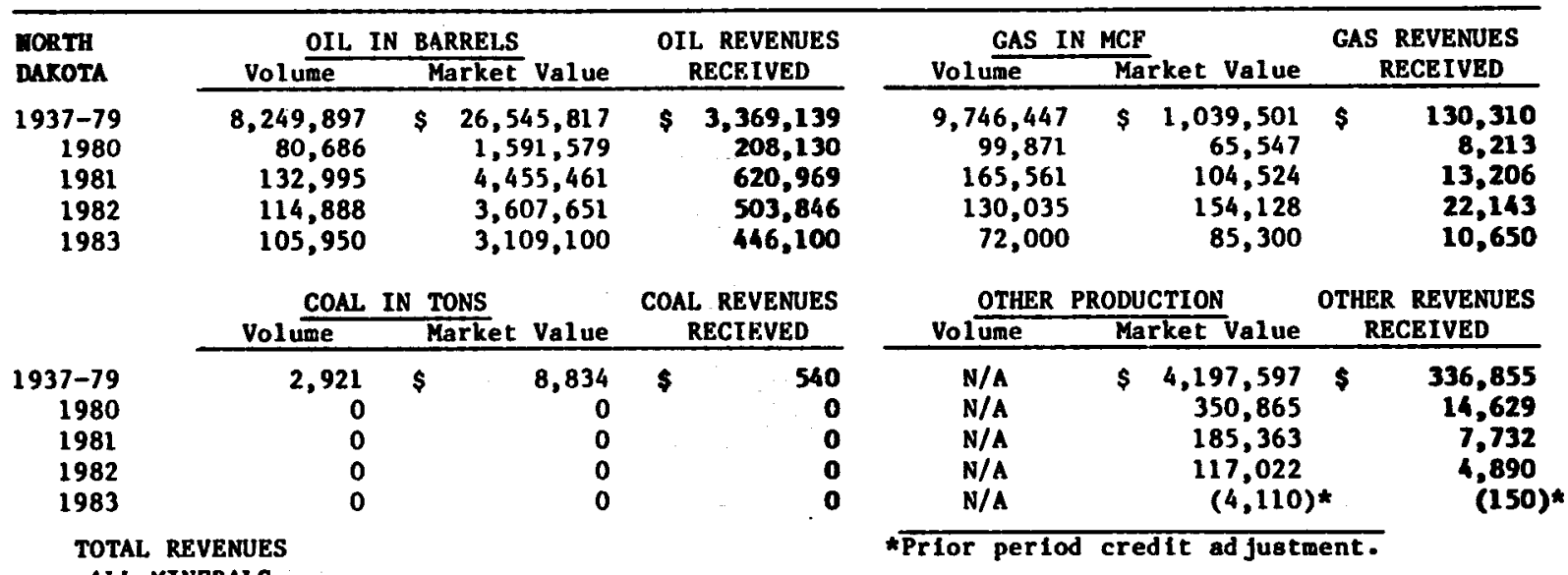

\begin{tabular}{rrr}
\multicolumn{2}{c}{ ALL } & MINERALS \\
\hline $1937-79$ & $\$ 3,836,844$ \\
1980 & & 230,972 \\
1981 & 641,907 \\
1982 & & 530,879 \\
1983 & & 456,600 \\
$1937-83$ & $\$ \quad 5,697,202$ \\
\hline
\end{tabular}


Table 14 (cont'd):

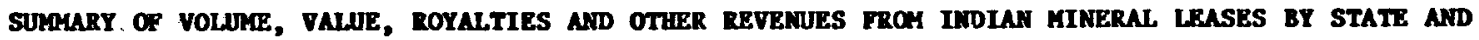
COMMODITY, 1937-83

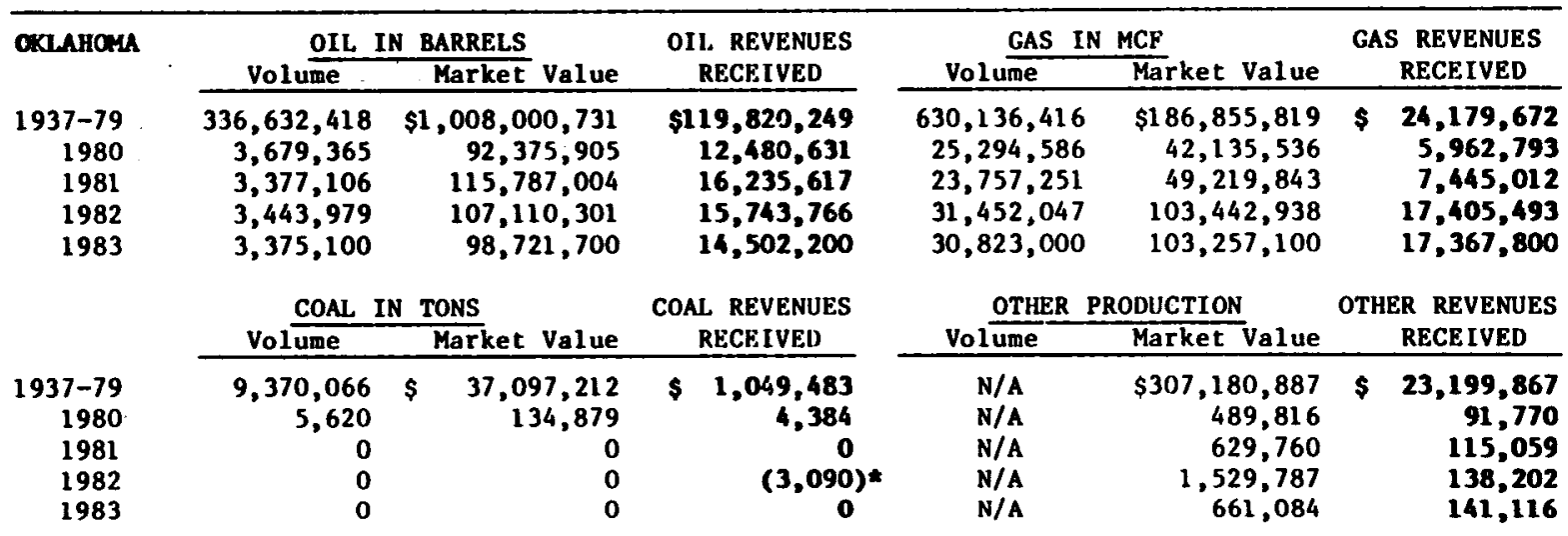

$\star \overline{\text { Prior period credit adjustment. }}$

TOTAL REVENUES

ALL MINERALS

\begin{tabular}{rr}
\hline $1937-79$ & $\$ 168,249,271$ \\
1980 & $18,539,578$ \\
1981 & $23,795,688$ \\
1982 & $33,284,371$ \\
1983 & $32,011,116$ \\
$1937-83$ & $\$ 275,880,024$ \\
\hline
\end{tabular}

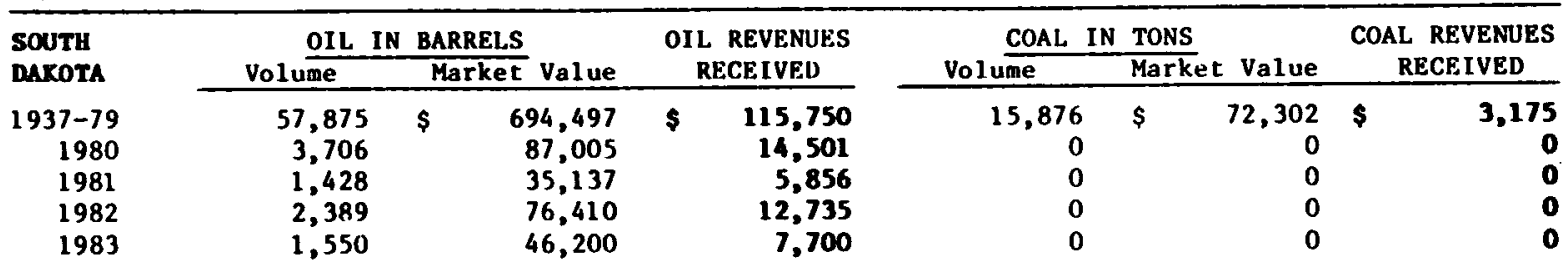

\begin{tabular}{|c|c|c|c|c|c|c|c|c|}
\hline \multirow[b]{2}{*}{$\begin{array}{r}1937-79 \\
1980 \\
1981 \\
1982 \\
1983\end{array}$} & \multirow{2}{*}{$\begin{array}{l}\text { OTHER } \\
\text { Volume } \\
\text { N/A } \\
\text { N/A } \\
\text { N/A } \\
\text { N/A } \\
\text { N/A }\end{array}$} & \multicolumn{2}{|c|}{$\frac{\text { PRODUCTION }}{\text { Market Value }}$} & \multicolumn{2}{|c|}{$\begin{array}{c}\text { OTHER REVENUES } \\
\text { RECEIVED } \\
\end{array}$} & \multicolumn{3}{|c|}{$\begin{array}{c}\text { TOTAL REVENUES } \\
\text { ALL MINERALS }\end{array}$} \\
\hline & & $\$$ & $\begin{array}{r}5,950,624 \\
0 \\
0 \\
0 \\
0\end{array}$ & $\$$ & $\begin{array}{r}474,609 \\
0 \\
0 \\
0 \\
0\end{array}$ & $\begin{array}{r}1937-79 \\
1980 \\
1981 \\
1982 \\
1983 \\
1937-83\end{array}$ & $\$$ & $\begin{array}{r}593,534 \\
14,501 \\
5,856 \\
12,735 \\
7,700 \\
634,326 \\
\end{array}$ \\
\hline
\end{tabular}


Table 14 (cont'd):

SURMARY OF VOLURR, VALUE, ROYALTIES AND OTHER REVEIUES FROM INDLAN GINERAL IRASES BY STATE AND COMODIT, 1937-83

\begin{tabular}{|c|c|c|c|c|c|c|c|}
\hline \multirow[t]{2}{*}{ UTAH } & \multicolumn{2}{|c|}{ OIL IN BARRELS } & OIL REVENUES & \multicolumn{2}{|c|}{ GAS IN MCF } & \multicolumn{2}{|c|}{ GAS REVENUES } \\
\hline & Volume & Market Value & RECEIVED & Volume & Market Value & & RECEIVED \\
\hline \multirow[t]{2}{*}{$\begin{array}{r}1937-79 \\
1980 \\
1981 \\
1982 \\
1983\end{array}$} & $\begin{array}{r}335,233,795 \\
9,597,679 \\
9,014,098 \\
8,274,857 \\
8,693,450\end{array}$ & $\begin{array}{r}\$ 1,375,753,961 \\
167,272,016 \\
308,136,794 \\
258,292,771 \\
274,283,900\end{array}$ & $\begin{array}{r}\$ 208,890,803 \\
26,573,970 \\
48,345,830 \\
41,436,094 \\
42,882,900\end{array}$ & $\begin{array}{r}229,391,406 \\
3,729,220 \\
7,847,505 \\
9,435,261 \\
6,753,000\end{array}$ & $\begin{array}{r}\$ 69,314,846 \\
18,243,023 \\
18,119,111 \\
27,793,618 \\
32,845,300\end{array}$ & $\$$ & $\begin{array}{r}10,626,408 \\
2,982,001 \\
2,965,454 \\
4,574,342 \\
5,467,500\end{array}$ \\
\hline & Volume & $\frac{\text { PRODUCTION }}{\text { Market Value }}$ & $\begin{array}{c}\text { OTHER REVENUES } \\
\text { RECEIVED } \\
\end{array}$ & $\begin{array}{c}\text { TOTAL } \\
\text { ALL } M \\
\end{array}$ & $\begin{array}{l}\text { LEVENUES } \\
\text { INERALS } \\
\end{array}$ & & \\
\hline $\begin{array}{r}1937-79 \\
1980 \\
1981 \\
1982 \\
1983\end{array}$ & $\begin{array}{l}N / A \\
N / A \\
N / A \\
N / A \\
N / A\end{array}$ & $\begin{array}{l}49,484,742 \\
18,370,253 \\
16,386,712 \\
44,484,658 \\
17,966,409\end{array}$ & $\begin{array}{r}\$ 2,607,153 \\
1,076,132 \\
895,026 \\
2,161,887 \\
996,595\end{array}$ & $\begin{array}{r}1937-79 \\
1980 \\
1981 \\
1982 \\
1983 \\
1937-83\end{array}$ & $\begin{array}{r}\$ 222,124,364 \\
30,632,103 \\
52,206,310 \\
48,172,323 \\
49,346,995 \\
\$ 402,482,095 \\
\end{array}$ & & \\
\hline
\end{tabular}

\begin{tabular}{|c|c|c|c|c|c|c|c|c|c|}
\hline \multirow[t]{2}{*}{ WASHINGTON } & \multicolumn{2}{|c|}{ OIL IN BARRELS } & & \multicolumn{2}{|c|}{ OIL REVENUES } & \multirow{2}{*}{ Volume } & PRODUCTION & \multirow{2}{*}{\multicolumn{2}{|c|}{$\begin{array}{c}\text { OTHER REVENUES } \\
\text { RECE IVED } \\
\end{array}$}} \\
\hline & Volume & Market & Value & & & & Market Value & & \\
\hline $\begin{array}{r}1937-79 \\
1980 \\
1981 \\
1982 \\
1983\end{array}$ & $\begin{array}{r}2,939 \\
0 \\
0 \\
0 \\
0\end{array}$ & $\$$ & $\begin{array}{r}1,170 \\
0 \\
0 \\
0 \\
0\end{array}$ & $\$$ & $\begin{array}{r}146 \\
0 \\
0 \\
0 \\
0\end{array}$ & $\begin{array}{l}N / A \\
N / A \\
N / A \\
N / A \\
N / A\end{array}$ & 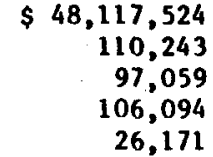 & $\$$ & $\begin{array}{r}4,917,858 \\
19,342 \\
19,613 \\
14,803 \\
16,500\end{array}$ \\
\hline
\end{tabular}

TOTAL REVENUES

\begin{tabular}{rr} 
ALL & MINERALS \\
\hline $1937-79$ & $\$ \quad 4,918,004$ \\
1980 & 19,342 \\
1981 & 19,613 \\
1982 & 14,803 \\
1983 & 16,500 \\
$1937-83$ & $\$ \quad 1,988,262$ \\
\hline
\end{tabular}

\begin{tabular}{|c|c|c|c|c|c|c|c|c|}
\hline \multirow{2}{*}{$\begin{array}{l}\text { UISCONSIN } \\
\begin{array}{r}1937-79 \\
1983\end{array}\end{array}$} & \multirow{2}{*}{$\frac{\text { OTHER }}{\text { Volume }}$} & $\begin{array}{c}\text { PRODUCTION } \\
\text { Market } \\
\end{array}$ & \multicolumn{3}{|c|}{$\begin{array}{cc}\mathrm{N} & \text { OTHER REVENUES } \\
\text { Val ue } & \text { RECEIVED } \\
\end{array}$} & \multicolumn{3}{|c|}{$\begin{array}{c}\text { TOTAL REVENUES } \\
\text { ALL MINERALS }\end{array}$} \\
\hline & & $\$$ & $\begin{array}{r}40,711 \\
0\end{array}$ & $\$$ & $\begin{array}{r}3,271 \\
0\end{array}$ & $\begin{array}{l}1937-79 \\
1980-83 \\
1937-83\end{array}$ & $\begin{array}{l}\$ \\
\$\end{array}$ & $\begin{array}{r}3,271 \\
0 \\
3,271 \\
\end{array}$ \\
\hline
\end{tabular}


Table 14 (cont'd):

SUNHARY OF VOLUK, VALUE, ROTALTIES AMD OTHER RBVENUES FROA IRDLAN MINERAL LEASES BT STATR AND COMODIT, 1937-83

\begin{tabular}{|c|c|c|c|c|c|c|c|}
\hline \multirow{3}{*}{$\begin{array}{r}\text { ryokIng } \\
1937-79 \\
1980 \\
1981 \\
1982 \\
1983\end{array}$} & \multicolumn{2}{|c|}{ OIL IN BARRELS } & OIL REVENUES & \multicolumn{2}{|c|}{ GAS IN MCF } & \multicolumn{2}{|c|}{ GAS REVENUES } \\
\hline & Volume & Market Value & RECE IVED & Volume & Market Value & & RECEIVED \\
\hline & $\begin{array}{r}214,541,430 \\
4,839,034 \\
5,744,444 \\
4,400,381 \\
4,498,000\end{array}$ & $\begin{array}{r}608,182,008 \\
80,592,737 \\
147,421,863 \\
123,715,600 \\
116,589,000\end{array}$ & $\begin{array}{r}\$ 81,221,735 \\
12,508,129 \\
21,289,668 \\
17,957,252 \\
16,911,000\end{array}$ & $\begin{array}{r}237,743,872 \\
12,885,366 \\
12,179,482 \\
15,378,204 \\
13,678,000\end{array}$ & $\begin{array}{r}59,949,366 \\
21,107,916 \\
23,144,537 \\
37,394,020 \\
35,275,000\end{array}$ & $\$$ & $\begin{array}{l}8,949,971 \\
3,385,668 \\
3,547,986 \\
5,544,353 \\
5,072,000\end{array}$ \\
\hline & OTHER & $\frac{\text { PRODUCTION }}{\text { Market Value }}$ & $\begin{array}{c}\text { OTHER REVENUES } \\
\text { RECEIVED }\end{array}$ & $\begin{array}{l}\text { TOTAL R } \\
\text { ALL MI }\end{array}$ & $\begin{array}{l}\text { VENUES } \\
\text { ERALS }\end{array}$ & & \\
\hline $\begin{array}{r}1937-79 \\
1980 \\
1981 \\
1982 \\
1983\end{array}$ & $\begin{array}{l}N / A \\
N / A \\
N / A \\
N / A \\
N / A\end{array}$ & $\begin{array}{r}3,444,167 \\
207,762 \\
545,441 \\
724,930 \\
1,017,000\end{array}$ & $\begin{array}{r}331,554 \\
24,018 \\
28,107 \\
30,818 \\
43,000\end{array}$ & $\begin{array}{r}1937-79 \\
1980 \\
1981 \\
1982 \\
1983 \\
1937-83\end{array}$ & $\begin{array}{r}\$ 90,413,622 \\
15,917,815 \\
24,865,761 \\
23,532,423 \\
22,026,000 \\
\$ 176,755,621 \\
\end{array}$ & & \\
\hline
\end{tabular}

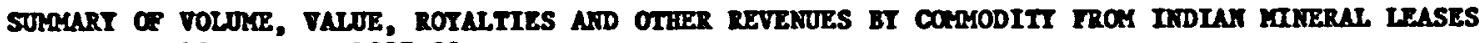
LOCATED IN 18 STATES, 1937-83

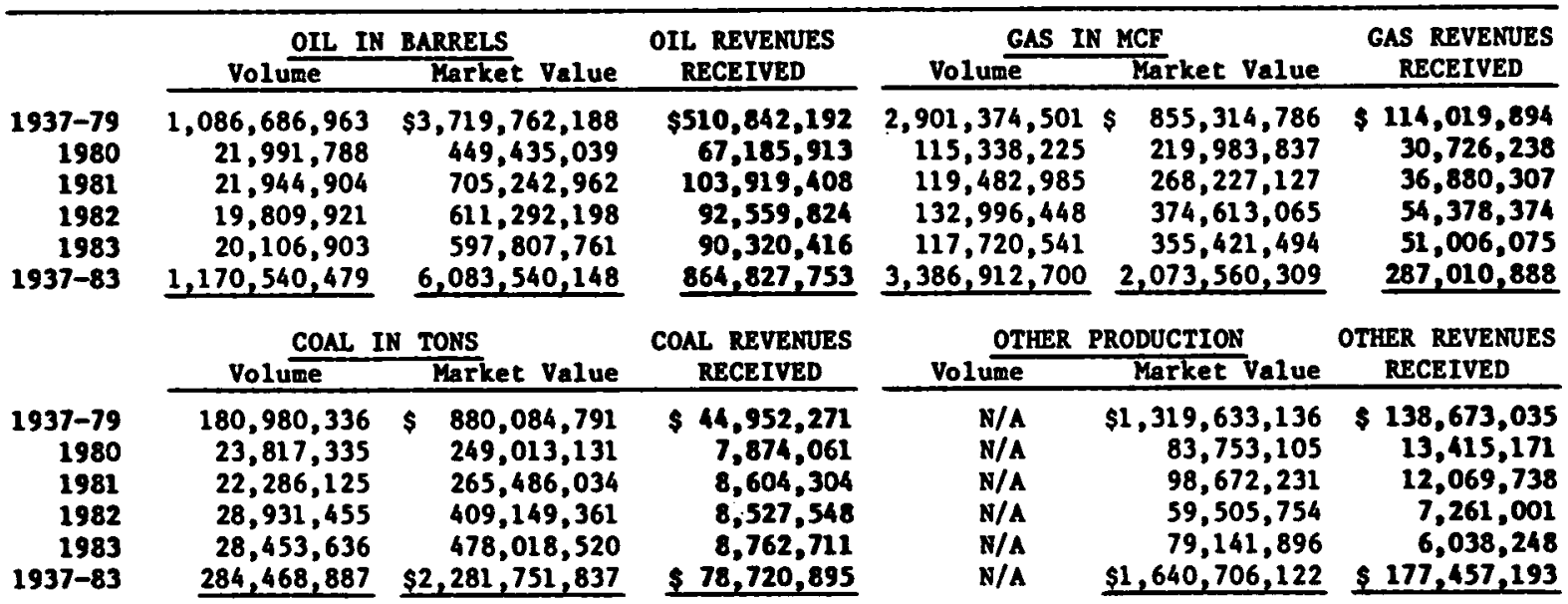

TOTAL REVENUES

ALL MINERALS

\begin{tabular}{rrr}
\hline $1937-79$ & $\$ 808,487,392$ \\
1980 & $119,201,383$ \\
1981 & $161,473,757$ \\
1982 & $162,726,747$ \\
1983 & $156,127,450$ \\
$1937-83$ & $\$ 1,408,016,729$ \\
\hline
\end{tabular}




\section{MIINERAL REVENUES}

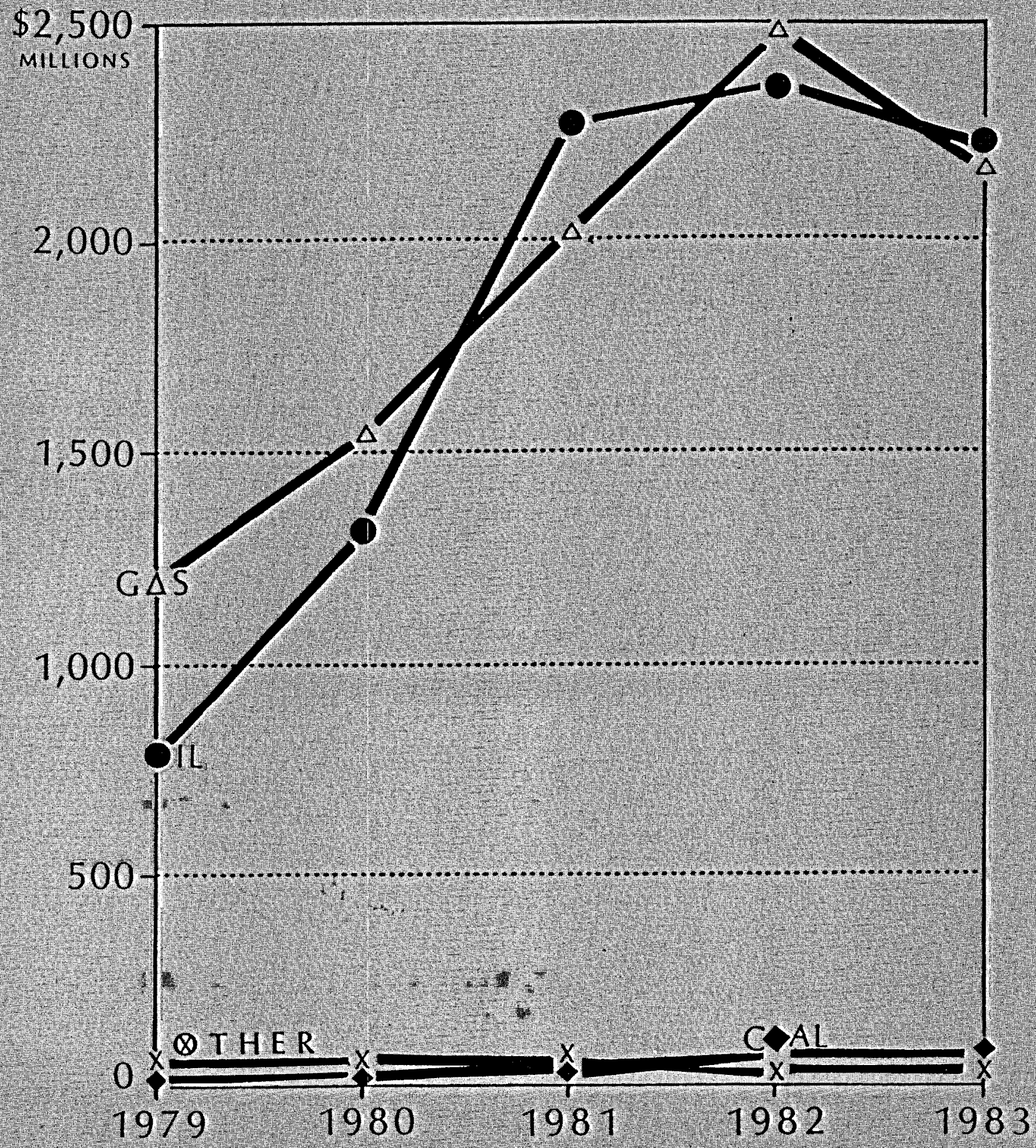




\section{DISTRIBUTION OF FEDERAL \& INDIAN MINERAL REVENUES}

All revenue collections from mineral leasing on Federal lands were distributed in 1983 by the Minerals Management Service and the Bureau of Land Management to the States, to the Federal Treasury and to its windfall profit tax account, and to various special-purpose accounts. All royalty revenues collected from Indian lands were distributed by the Bureau of Indian Affairs to the appropriate Indian Tribes and Allottees. The formulas for these disbursements are established by law.

Last year, 76.1 percent of all mineral revenues went to the U.S. Treasury in addition to the 5.4 percent paid as windfall profit tax on oil extracted from Federal leases (Table 1-b). Another 4.3 percent of the total 1983 revenues were distributed directly to States and 12.5 percent to earmarked funds created by law for specific purposes. All royalty revenues from Indian leases, representing 1.7 percent of all $1983 \mathrm{Federal}$ and Indian revenues, were distributed entirely to tribal governments and individual allotment owners.

Ftgure 8 :

1983 DISBURSEKENT OF HIKERAL REVENUES IN MILLIOHS TROH FEDERAL AMD IMDIAH MINERAL LEASES

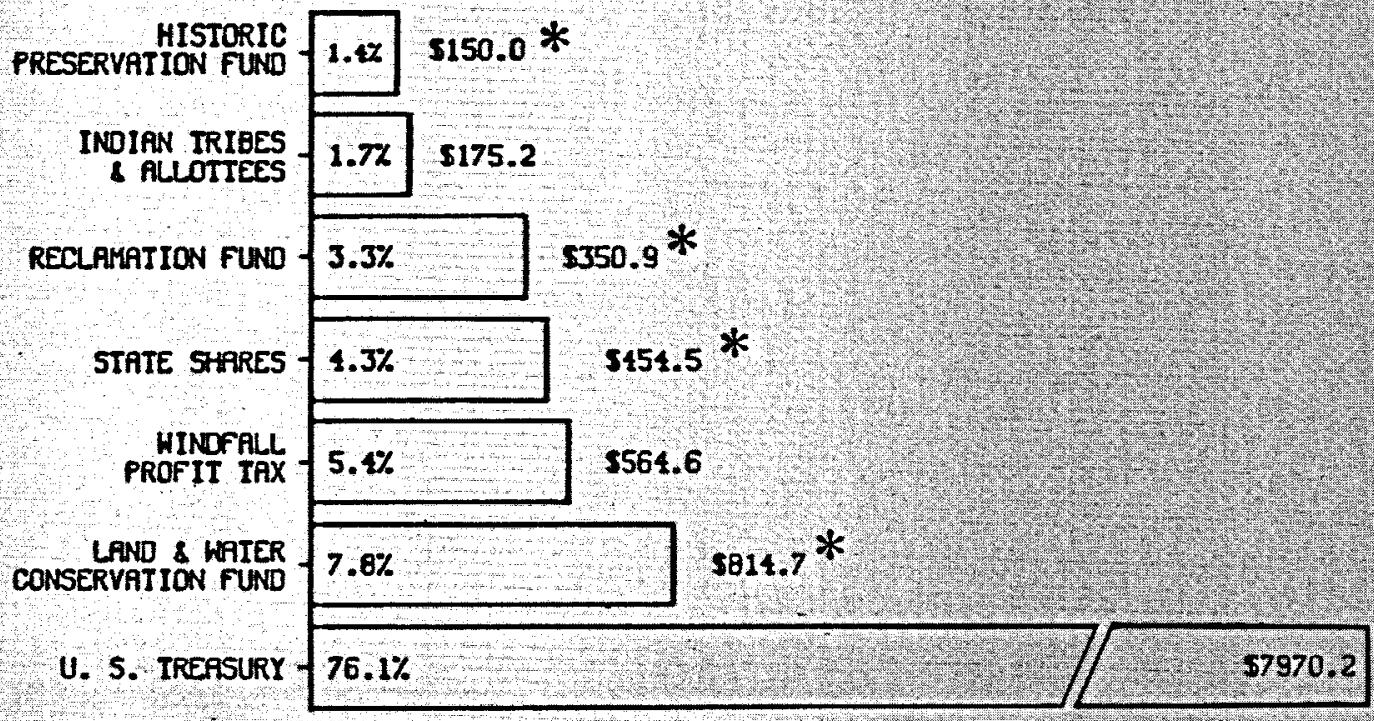

* Fiscal year payments; 11 others by calendar year. 
The Mineral Leasing Act of February 25, 1920 provides that States whose boundaries encompass Federal mineral leases will share the revenues with the Federal Government. Accordingly, in 1983, 26 States shared $\$ 454,359,341$ in disbursements of bonus, rental, and royalty revenues ( $T$ able 16). Of these, 5 mineral-producing States (Wyoming, New Mexico, Colorado, Utah, and California) received 84 percent of that money; 21 States received the other 16 percent. Wyoming's share was $\$ 168.2$ million or 37 percent of the total disbursed to the States. The market value of the oil, gas, coal, and other minerals produced from Federal leases in Wyoming was $\$ 3.8$ billion alone. New Mexico, the recipient of the second largest share, collected $\$ 119$ million or 26 percent of the $\$ 454.4$ million.

The periodic disbursement of bonuses, rents, and royalty income is governed by legislation and regulations. Historically, payments to State governments had been made twice each fiscal year, generally in May and November. However, those State shares of mineral revenues collected after October 1, 1983, are being disbursed on a monthly basis, as prescribed in the Federal Oil and Gas Royalty Management Act of 1982.

Tribal and allottee revenues are processed and passed through to the Bureau of Indian Affairs for disbursement to Indian accounts as rapidly as possible af ter receipt.

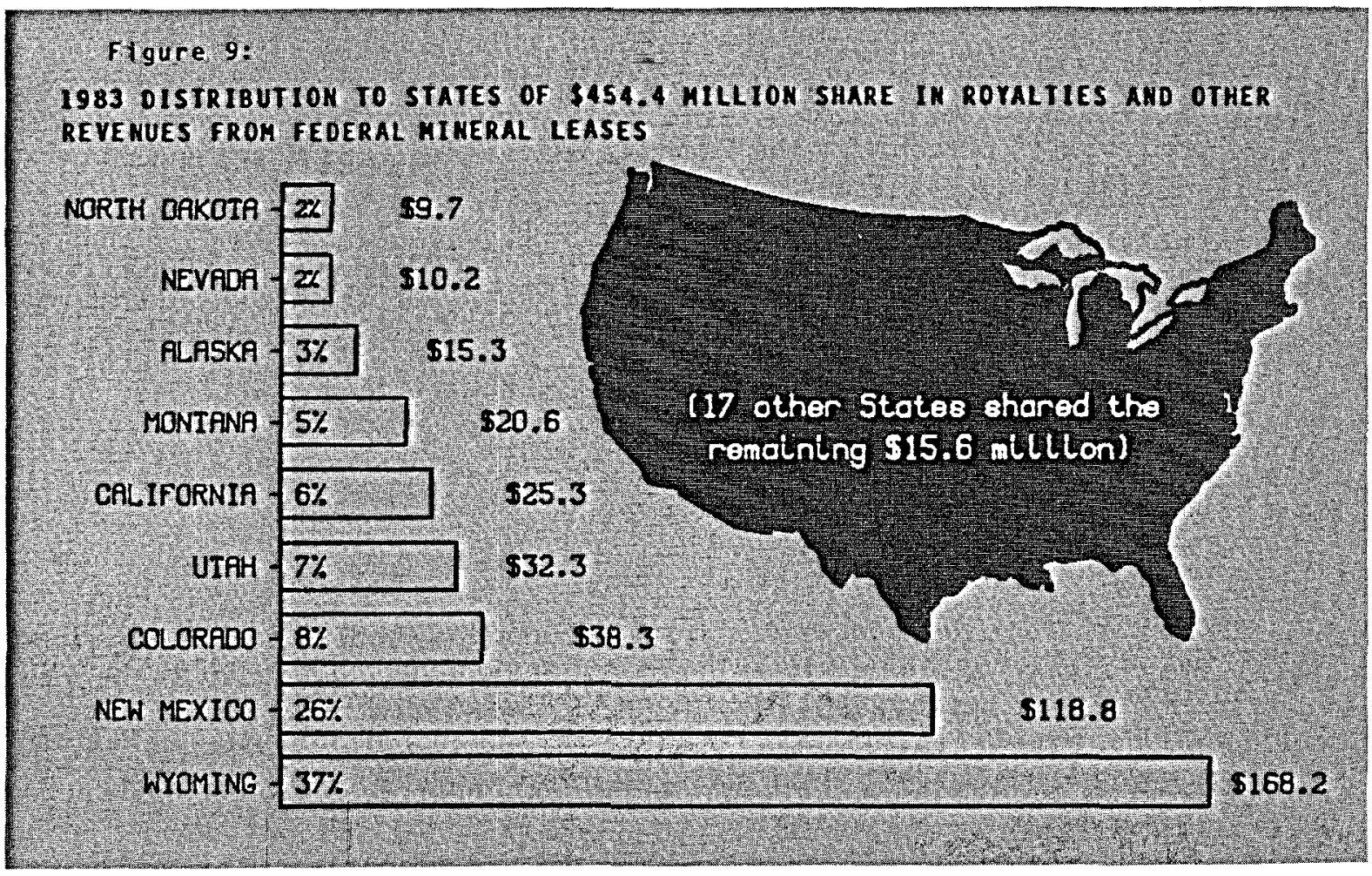




\section{Public Lands Leases}

The royalty revenues collected from public lands leases are distributed to the States, various funds, and the general fund of the Treasury.

All States, excepting Alaska, receive 50 percent of all rents, bonuses, and royalties collected from any public lands leases located within a State's boundaries. These revenues do not include the windfall profit tax on crude oil, which is deducted before any allocation is made. Of the remaining mineral revenues from public lands leases, 40 percent are deposited in the Reclamation Fund and 10 percent in the general fund of the Treasury.

Alaska receives 90 percent of all rents, bonuses, and royalties paid for mineral production on public lands leases after deduction of payments and escrows paid under the provisions of the Alaska Native Claims Settlement Act of 1971 and after deduction of windfall profit tax on oil when appropriate. The State also receives 50 percent of all rents, bonuses, and royalties collected from the National Petroleum Reserve of Alaska.

\section{Acquired Lands Leases}

All rents, royalties, and bonuses for leases issued under the 1947 Mineral Leasing Act for Acquired Lands are collected by MMS on behalf of the U.S. Forest Service, Department of Agriculture, and disbursed as mandated.

Royalty revenues from leases within a national forest are distributed as follows: 25 percent for the $\mathrm{State}$ in which the mineral resource is produced; 10 percent for the Forests, Roads, and Trails Fund; and 65 percent for the general Treasury fund.

Royalty revenues from leases within a national grassland are distributed as follows 25 percent for the county in which the mineral resource is produced; 10 percent for the Forests, Roads, and Trails Fund; and 65 percent for the general Treasury fund.

Receipts from other acquired lands are deposited in a general Treasury account. In some instances the funds revert to the agency that has administrative jurisdiction over the land.

\section{Indian Lands Leases}

Royalties and rentals from Indian tribal and allottee land leases are disbursed to the Bureau of Indian Affairs which then distributes them to the Indian Tribes, Indian Nations, and Allottees (individual Indians). revenues.

The Osage Nation manages its own leases and collects its own 


\section{Military Lands Leases}

All royalty revenues from military lands leases issued before 1981 are collected for deposit in the general fund of the Treasury. For leases issued since January 1,1981 , all royalty revenues collected are distributed in the same manner as revenues from public lands leases. The 1983 revenues from all military lands leases amounted to $\$ 4,578,452$.

\section{Outer Continental Shelf Lands Leases}

Royalty revenues from offshore leases in the Outer Continental Shelf (OCS) lands are distributed to two special funds and the general fund of the Treasury. In order to maintain the Land and Water Conservation Fund at $\$ 900$ million, transfers are made in each fiscal year from rents, bonuses, and royalties. These transfers amounted to $\$ 855$ million in $F Y$ 1982 and to $\$ 815$ million in FY 1983. Transfers to the Historic Preservation Fund from rents, bonuses, and royalties amounted to $\$ 150$ million in FY 1982 and to $\$ 150$ million in FY 1983.

The balance of offshore royalties go into the windfall profit tax account and the general fund of the Treasury.

Table 15:

1983 ROTALTIES, RENTS, AKD BONUSES FROM MTKRRAL IEASES IN FEDERAL AND INDIAN IANDS

\begin{tabular}{|c|c|c|c|c|c|}
\hline & \multicolumn{3}{|c|}{ FEDERAL LANDS } & \multirow{2}{*}{$\begin{array}{l}\text { INDIAN LANDS } \\
\text { In } 18 \text { states }\end{array}$} & \multirow{2}{*}{$\begin{array}{c}\text { TOTAL } \\
\text { REVENUES }\end{array}$} \\
\hline & Offshore & & Onshore & & \\
\hline Onl Royalties & $\$ 1,603,942,838$ & $\$$ & $522,661,131$ & $\$ 90,320,416$ & $\$ 2,216,924,385$ \\
\hline Gas Royalties & $1,771,745,327$ & & $348,616,847$ & $51,006,075$ & $2,171,368,249$ \\
\hline Coal Royalties & $N / A$ & & $56,666,428$ & $8,762,711$ & $65,429,139$ \\
\hline Other Rojalties & $\mathbf{N} / \mathbf{A}$ & & $39,906,641$ & $6,038,248$ & $45,944,889$ \\
\hline Winfmum Royalties & $4,463,658$ & & $1,446,721$ & $\mathbf{N} / \mathbf{A}$ & $5,910,379$ \\
\hline Rents & $32,267,343$ & & $128,065,207$ & $3,621,550$ & $163,954,100$ \\
\hline Bonuses & $5,749,016,369$ & & $62,108,205 *$ & $15,418,356$ & $5,826,542,930$ \\
\hline
\end{tabular}

T 0 T A L:

$\$ 9,161,435,53$

$\$ 1,159,471,180$

$\$ 175,167,356$

$\$ 10,496,074,071$

*Fiscal 1983 data In Public Land Stat1stics, 1983, Bureau of Land Management.

more: "Other Royalties" are collected for certain of the solld and fluid minerals footnoted In Table 2 .

Minimum royalties, by terms of the lease, may be collected at the discretion of the Secretary of the Interfor for an offshore Federal lease after the explration of the lease year that commences after the discovery of paying quantities. 
Table 16:

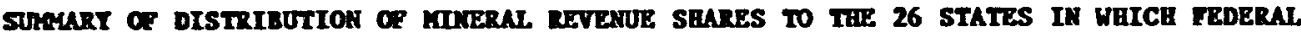
MTKERAT LEASES ARE LOCATED, 1920-83

\begin{tabular}{|c|c|c|c|c|c|c|c|}
\hline & \multicolumn{6}{|c|}{ STATE S } \\
\hline & & $1920-79$ & 1980 & 1981 & 1982 & 1983 & $1920-83$ \\
\hline $\begin{array}{l}1 . \\
2 . \\
3 . \\
4 . \\
5 . \\
6 . \\
7 . \\
8 . \\
9 . \\
10 . \\
11 . \\
12 . \\
13 . \\
14 . \\
15 . \\
16 . \\
17 . \\
18 . \\
19 . \\
20 . \\
21 . \\
22 . \\
23 . \\
24 . \\
25 . \\
26 .\end{array}$ & 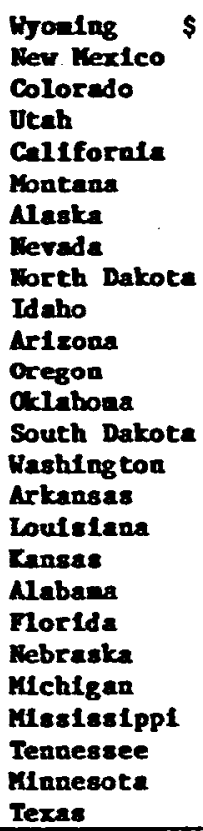 & $\begin{array}{r}659,558 \\
471,578 \\
238,292 \\
117,147 \\
158,492 \\
84,572 \\
128,198 \\
23,598 \\
8,599 \\
12,877 \\
6,153 \\
1,329 \\
9,083 \\
4,522 \\
258 \\
1,422 \\
6,565 \\
5,112 \\
275 \\
203 \\
377 \\
242 \\
239 \\
-- \\
-- \\
\end{array}$ & $\begin{array}{r}\$ 115,062 \\
105,081 \\
21,306 \\
17,028 \\
23,788 \\
9,954 \\
2,497 \\
7,187 \\
4,229 \\
2,895 \\
2,005 \\
306 \\
1,594 \\
738 \\
36 \\
236 \\
355 \\
891 \\
38 \\
27 \\
184 \\
32 \\
31 \\
- \\
- \\
\end{array}$ & $\begin{array}{r}\$ 119,956 \\
128,127 \\
24,939 \\
21,391 \\
24,634 \\
13,110 \\
18,865 \\
10,291 \\
4,094 \\
3,284 \\
4,802 \\
1,084 \\
2,238 \\
751 \\
48 \\
283 \\
612 \\
669 \\
36 \\
165 \\
148 \\
54 \\
39 \\
--- \\
--- \\
--- \\
\end{array}$ & $\begin{array}{r}\$ 172,096 \\
146,813 \\
42,807 \\
80,916 \\
35,440 \\
18,024 \\
44,380 \\
12,595 \\
7,007 \\
5,015 \\
4,872 \\
4,416 \\
2,211 \\
1,280 \\
583 \\
21,614 \\
617 \\
1,022 \\
223 \\
17 \\
220 \\
70 \\
7,422 \\
-\cdots \\
--- \\
\end{array}$ & 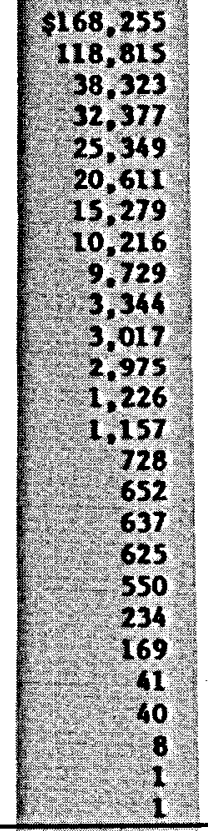 & $\begin{array}{r}\$ 1,234,927 \\
970,414 \\
365,667 \\
268,859 \\
267,703 \\
146,271 \\
209,219 \\
63,887 \\
33,658 \\
27,415 \\
20,849 \\
10,110 \\
16,352 \\
8,448 \\
1,653 \\
24,207 \\
8,786 \\
8,319 \\
1,122 \\
646 \\
1,098 \\
439 \\
7,771 \\
8 \star \\
1 \star \\
1 \star \\
\end{array}$ \\
\hline & O TAL: & 938,691 & $\$ 315,500$ & $\$ 379,620$ & $\$ 609,660$ & $\$ 454,359$ & $\$ 3,697,830$ \\
\hline
\end{tabular}

*Cumulative totals for these and certain other States do not necessarily include some very small revenue shares that may have been disbursed during the prevlous 62 years.

motz: Mandated distribution to the States is based on percentage shares in bonuses, rents, royalties, and other wineral revenue collections. The States are ranked here by their 1983 shares of Federal mineral revenue collectlons.

Included In the above 1983 State shares, from the Natlonal Petroleum Reserve: $\$ 1,649,475$ for Mlaska; from acquired military lands: $\$ 411,687$ for Artansas, $\$ 1,950$ for Callfornia, $\$ 14,870$ for Mortda, $\$ 957$ for Oregon, $\$ 15,601$ for Tennessee, $\$ 1,002$ for Texas; from jolnt Pedera1-State audits of delinquent royalty payors: $\$ 2,171,069$ for colorado, $\$ 290,228$ for Hontana, $\$ 35,853$ for Nevada, $\$ 198,095$ for Dorth Dakota, $\$ 1,233$ for vtah, $\$ 5,357,303$ for Hyontag.

SOURCE: Public Land Statist lcs, 1983, Bureau of Land Management. 
Table 17:

SUMAARY OF INDIAN ROTALTIES AND OTHER MINFRAL RECEIPTS BY YEAR AND COMMODITY, 1937-83

\begin{tabular}{|c|c|c|c|c|c|}
\hline & $\begin{array}{c}\text { OIL REVENUES } \\
\text { RECEIVED } \\
\end{array}$ & $\begin{array}{l}\text { GAS REVENUES } \\
\text { RECE IVED } \\
\end{array}$ & $\begin{array}{c}\text { COAL REVENUES } \\
\text { RECE IVED } \\
\end{array}$ & $\begin{array}{c}\text { OTHER REVENIIES } \\
\text { RECEIVED } \\
\end{array}$ & $\begin{array}{r}\text { TOTAL, RFVENIISS } \\
\text { ALI. MI NERALIS }\end{array}$ \\
\hline $\begin{array}{r}1937-79 \\
1980 \\
1981 \\
1982 \\
1983 \\
1937-83\end{array}$ & $\begin{array}{r}\$ 510,842,192 \\
67,185,913 \\
103,919,408 \\
92,559,824 \\
90,320,416 \\
\$ 864,827,753 \\
\end{array}$ & $\begin{array}{r}\$ 114,019,894 \\
30,726,238 \\
36,880,307 \\
54,378,374 \\
51,006,075 \\
\$ 287,010,888 \\
\end{array}$ & $\begin{array}{r}\$ 44,952,271 \\
7,874,061 \\
8,604,304 \\
8,527,548 \\
8,762,711 \\
\$ 78,720,895 \\
\end{array}$ & $\begin{array}{r}\$ 138,673,035 \\
13,415,171 \\
12,069,738 \\
7,261,001 \\
6,038,248 \\
\$ 177,457,193 \\
\end{array}$ & $\begin{array}{r}808,487,392 \\
119,201,383 \\
161,473,757 \\
162,726,747 \\
156,127,450 \\
\$ 1,408,016,729 \\
\end{array}$ \\
\hline
\end{tabular}

BOTR: "Other Revenues" are collected for certain of the solid and fluid minerals footnoted in Table 2.

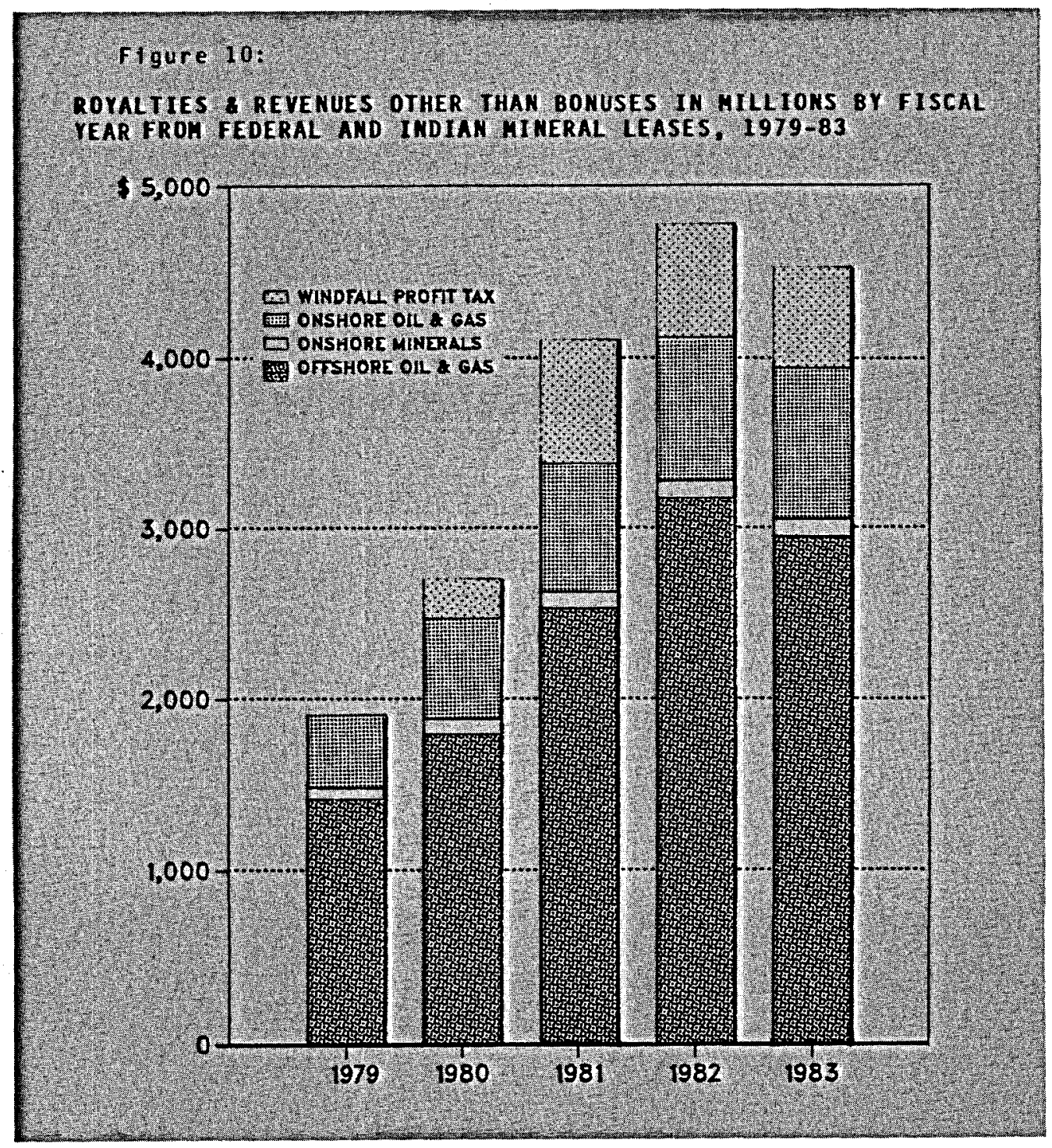




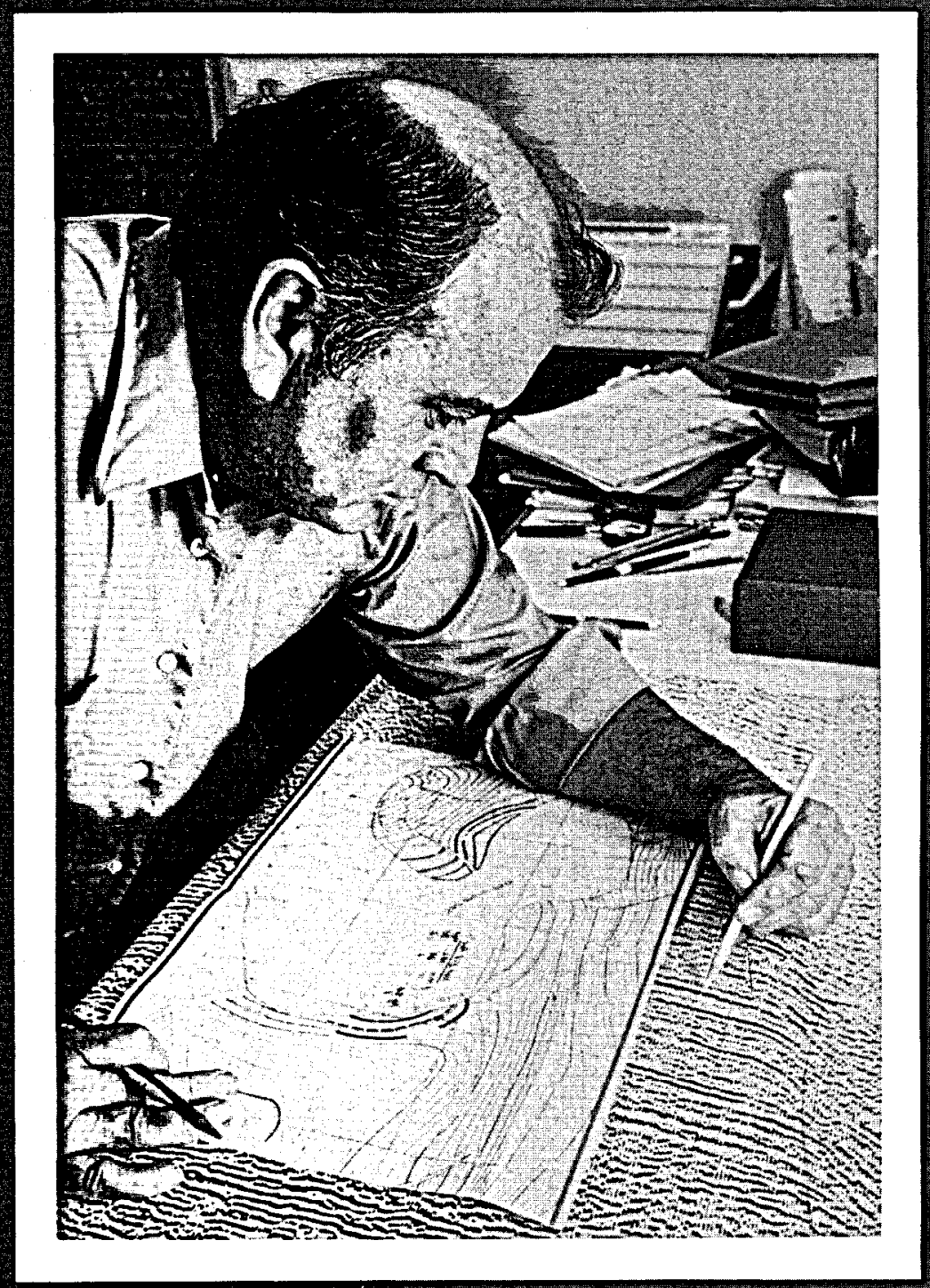

Resource evaluation, MS geologist. 


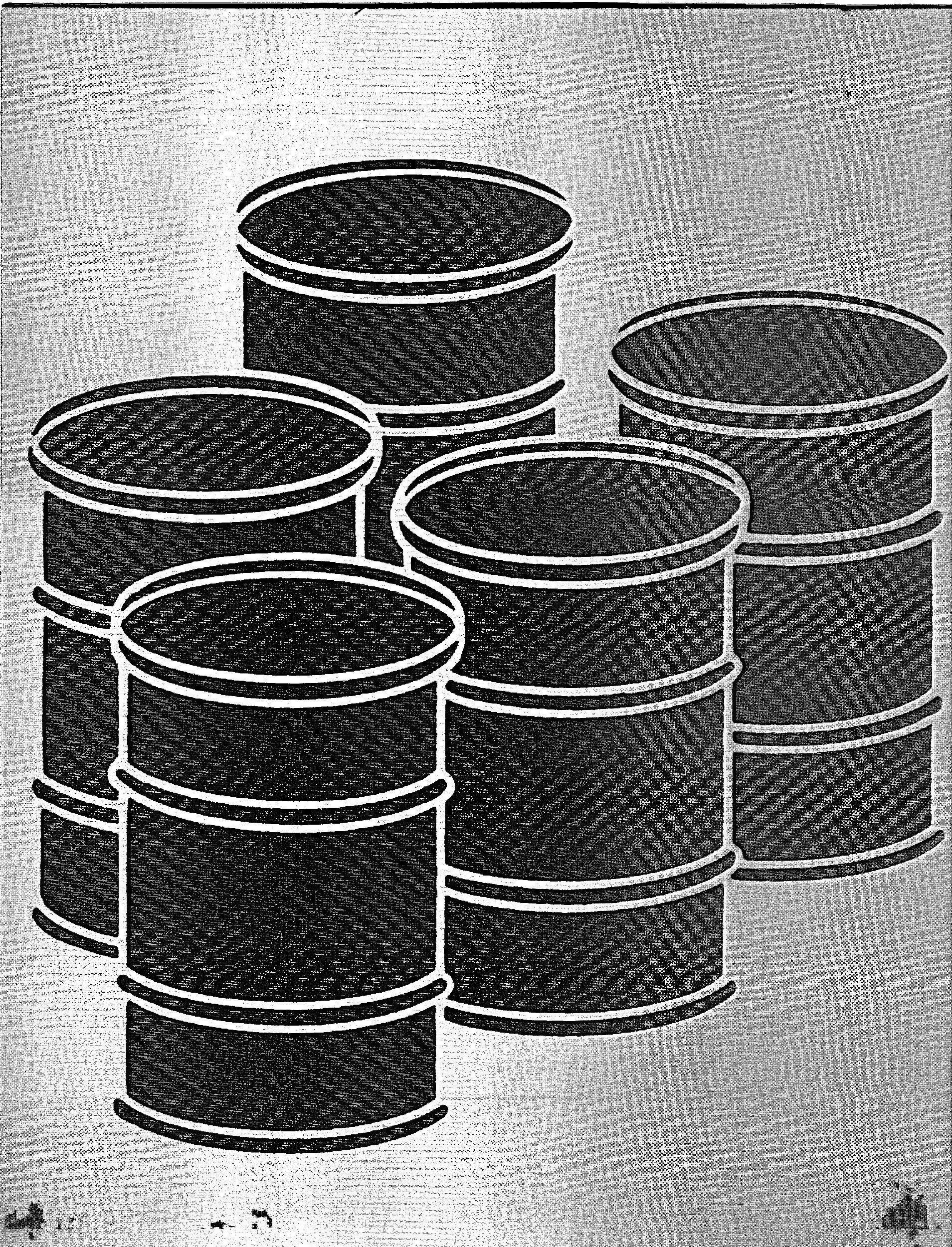


APPENDIX : 57

Table 18:

SUMAAT OR ROYALTY-IN-KIND OIL TAREN AS ROYAITY PATRENTS FOR FEDERAL LEASE PRODOCTION, 1976-83

\begin{tabular}{|c|c|c|c|c|c|c|}
\hline & \multicolumn{2}{|c|}{ ONSHORE RIK OIL } & \multicolumn{2}{|c|}{ OFFSHORE RIK OIL } & \multicolumn{2}{|c|}{ TOTAL RIK OIL } \\
\hline & Barrels Sold & Royalty Value & Barrels Sold & Royalty Value & Barrels Sold & Royalty Value \\
\hline $\begin{array}{r}1976-79 \\
1980 \\
1981 \\
1982 \\
1983 \\
1976-83\end{array}$ & $\begin{array}{r}45,009,893 \\
9,883,818 \\
7,498,508 \\
4,903,877 \\
6,912,354 \\
74,208,450 \\
\end{array}$ & $\begin{array}{r}371,148,985 \\
199,149,683 \\
240,535,887 \\
130,817,540 \\
191,886,947 \\
\$ 1,133,539,042 \\
\end{array}$ & $\begin{array}{r}109,443,406 \\
26,243,154 \\
22,086,347 \\
12,393,000 \\
6,582,569 \\
176,748,476 \\
\end{array}$ & $\begin{array}{r}\$ 1,000,428,731 \\
490,562,695 \\
761,461,463 \\
361,201,000 \\
185,891,749 \\
\$ 2,799,545,638 \\
\end{array}$ & $\begin{array}{r}154,453,299 \\
36,126,972 \\
29,584,855 \\
17,296,877 \\
13,494,923 \\
250,956,926 \\
\end{array}$ & $\begin{array}{r}\$ 1,371,577,716 \\
689,712,378 \\
1,001,997,350 \\
492,018,540 \\
377,778,696 \\
\$ 3,933,084,680 \\
\end{array}$ \\
\hline
\end{tabular}

HOTE: In mineral leases 1 ssued for Federal lands, the Federal Government reserves the right to take royalty-in-kind payment from lessees. RIK oll thus taken is sold periodically to crude-deficlent refiners legally eligible to purchase $1 t$.

Under both the Mineral Leasing Act of February 25, 1920 and the Outer Continental Shelf Lands Act of 1953, the Federal Government as lessor may take part or all of its oil and gas royalties "in kind." Historically, the Secretary of the Interior, exercising discretionary authority, has found that certain independent oil refiners are chronically crude deficient. Thus, Federal lessees can be directed to pay their royalty share in crude oil, rather than "in value" as cash. The Federal Government in turn sells this royalty-in-kind oil to eligible refiners. Since 1946, the Royalty-In-Kind (RIK) program has been highly successful in providing a steady, secure source of domestic crude to independent refiners who of ten have difficulty finding regular supplies of crude for their refining operations.

RIK crude is sold mainly to refiners with a refining capacity of less than 50,000 barrels per day. Contracts with these refiners are ordinarily made for a period of three years, and new ones were issued to 45 different refiners late in the year.

In 1983, 17 percent of all oil royalties were taken in kind by the Federal Government. Amounting to 13.5 million barrels, this royalty oil was worth $\$ 378$ million. Of crude oil taken in kind last year, 6.9 million barrels were produced onshore $(51$ percent) and the remaining 6.6 million (49 percent) were produced from the Outer Continental Shelf.

In 1983 the Department held 3 royalty oil sales: 2 of onshore and 1 of off shore royalty barrels. The resulting contracts, effective in 1984, will account for approximately $44 \%$ of all oil royalties to be paid to the Federal Government. 
Table 19:

PRODUCING AND PRODOCIBLE FEDERAL AND IRDIAN OIL \& GAS LEASES ONSBORE IN 26 STATES AND OFFSHORE IN 4 OCS AREAS, AS OF DECEMBER 31, 1983

\begin{tabular}{|c|c|c|c|c|c|c|}
\hline & \multicolumn{2}{|c|}{ INDIAN } & \multicolumn{2}{|c|}{ FEDERAL } & \multicolumn{2}{|c|}{ TOTAL ONSHORE } \\
\hline & No. & Acres & No. & Acres & No. & Acres \\
\hline Alabama & -- & -- & 10 & 1,196 & 10 & 1,196 \\
\hline Alaska & -- & -- & 35 & 63,481 & 35 & 63,481 \\
\hline Arizona & 13 & 22,595 & $-\infty$ & -- & 13 & 22,595 \\
\hline Arkansas & -- & -- & 103 & 55,670 & 103 & 55,670 \\
\hline California & -- & -- & 350 & 241,107 & 350 & 241,107 \\
\hline Colorado & 106 & 147,023 & 1,223 & 875,911 & 1,329 & $1,022,934$ \\
\hline I11ino1s & --- & -- & 2 & 20 & 2 & 20 \\
\hline Kansas & -- & --- & 181 & 54,419 & 181 & 54,419 \\
\hline Rentucky & --- & -- & 7 & 9,678 & 7 & 9,678 \\
\hline Louts lana & --- & --- & 100 & 55,212 & 100 & 55,212 \\
\hline Maryland & --- & -- & 3 & 3,763 & 3 & 3,763 \\
\hline Michigan & 1 & 18 & 17 & 9,040 & 18 & 9,058 \\
\hline Mississippi & -- & -- & 100 & 47,460 & 100 & 47,460 \\
\hline Montana & 438 & 109,580 & 1,246 & 757,737 & 1,684 & 867,317 \\
\hline Nebraska & --- & -- & 18 & 6,590 & 18 & 6,590 \\
\hline Nevada & -- & -- & 41 & 11,925 & 41 & 11,925 \\
\hline New Mexico & 472 & 491,610 & 5,103 & $3,045,470$ & 5,575 & $3,537,080$ \\
\hline North Dakota & 24 & 5,132 & 382 & 322,556 & 406 & 327,688 \\
\hline Ohio & --- & -- & 17 & 4,769 & 17 & 4,769 \\
\hline Oklahoma & 1,834 & 170,014 & 537 & 88,337 & 2,371 & 258,351 \\
\hline South Dakota & 3 & 739 & 40 & 94,031 & 43 & 94,770 \\
\hline Texas & -- & -- & 48 & 22,092 & 48 & 22,092 \\
\hline Utah & 869 & 232,950 & 856 & 897,747 & 1,725 & $1,130,697$ \\
\hline Virginia & --- & -- & 1 & 2,168 & 1 & 2,168 \\
\hline West Virginia & $-\infty$ & --- & 21 & 27,618 & 21 & 27,618 \\
\hline Wyoming & 105 & 56,901 & 5,030 & $2,750,367$ & 5,135 & $2,807,268$ \\
\hline SUBTOTAL: & 3,865 & $1,236,562$ & 15,471 & $9,448,364$ & 19,336 & $10,684,926$ \\
\hline
\end{tabular}

TOTAL OFFSHORE

\begin{tabular}{|c|c|c|c|c|}
\hline & & \\
\hline & No. & Acres & No. & Acres \\
\hline $\begin{array}{l}\text { Alaska OCS Area } \\
\text { California OCS Area } \\
\text { Louis lana OCS Area } \\
\text { Texas OCS Area }\end{array}$ & $\begin{array}{r}1 \\
35 \\
1,110 \\
235 \\
\end{array}$ & $\begin{array}{r}4,172 \\
176,776 \\
4,861,499 \\
1,236,906 \\
\end{array}$ & $\begin{array}{r}1 \\
35 \\
1,110 \\
235 \\
\end{array}$ & $\begin{array}{r}4,172 \\
176,776 \\
4,861,499 \\
1,236,906\end{array}$ \\
\hline SUBTOTAL: & 1,381 & $6,279,353$ & 1,381 & $6,279,353$ \\
\hline TOTAL: & 16,852 & $15,727,717$ & 20,717 & $16,964,279$ \\
\hline
\end{tabular}


Table 20:

PRODUCING FEDERAL AND ILDIAN COAL LEASES ONSHORE IN 10 STATES, AS OF DECEMBER 31, 1983

\begin{tabular}{|c|c|c|c|c|c|c|}
\hline & \multicolumn{2}{|c|}{ FEDERAL } & \multicolumn{2}{|c|}{ INDIAN } & \multicolumn{2}{|c|}{ TOTAL } \\
\hline & No. & Acres & No. & Acres & No. & Acres \\
\hline Alabama & 1 & 2,388 & - & -- & 1 & 2,388 \\
\hline Artzona & $-\infty$ & - & 2 & 64,858 & 2 & 64,858 \\
\hline Colorado & 26 & 23,927 & - & - & 26 & 23,927 \\
\hline Kontana & 10 & 23,535 & 1 & 14,746 & 11 & 38,281 \\
\hline New Mexico & 4 & 10,031 & 3 & 76,314 & 7 & 86,345 \\
\hline North Dakota & 3 & 2,601 & -- & - & 3 & 2,601 \\
\hline Oklahoma & 1 & 140 & - & - & 1 & 140 \\
\hline Utah & 30 & 34,911 & - & -- & 30 & 34,911 \\
\hline Washington & 1 & 241 & - & -- & 1 & 241 \\
\hline Wyouling & 27 & 76,584 & - & -- & 27 & 76,584 \\
\hline $\mathbf{T} O \mathbf{T A} \mathbf{L}$ & 103 & 174,358 & 6 & 155,918 & 109 & 330,276 \\
\hline
\end{tabular}


Table 21:

PRODOCING FEDERAL AND INDIAR LEASES OF OTHER MINERALS ONSHORE IN 18 STATES, AS OF DECEABER 31, 1983

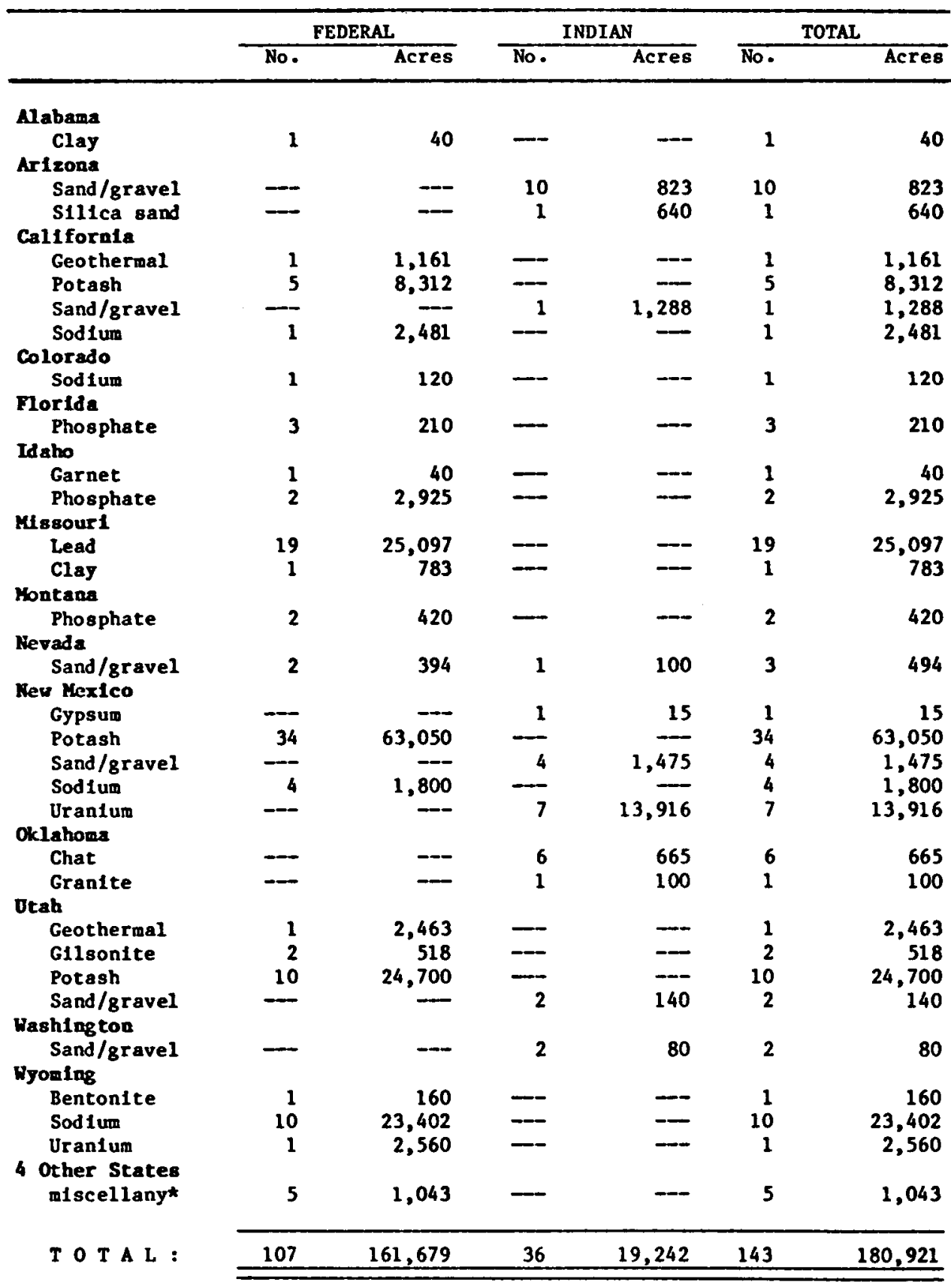

*Includes 2 leases for Arkanaas (quartz crystals) and 1 each for Ceorgia (feldspar), Minnesota (Iron ore), and Virginia (limestone). 
Table 22:

SUMAMARY OF OIL G GS AND OTHER MTHERAL IEASES, LICENSES, AND PERMITS UNDER THE SUPERVISION OP THE DEPARTMENT OP THE INTERIOR IN THE OFFSHORE FEDERAL LANDS, BY OCS AREA, AS OF DECEMBER 31, 1983

FEDERAL LEASES OFFSHORE

Location

offshore:

\section{Alabama}

Alaska

California

Florida

Louts lana

Texas

Atlantic States

T O T A L:

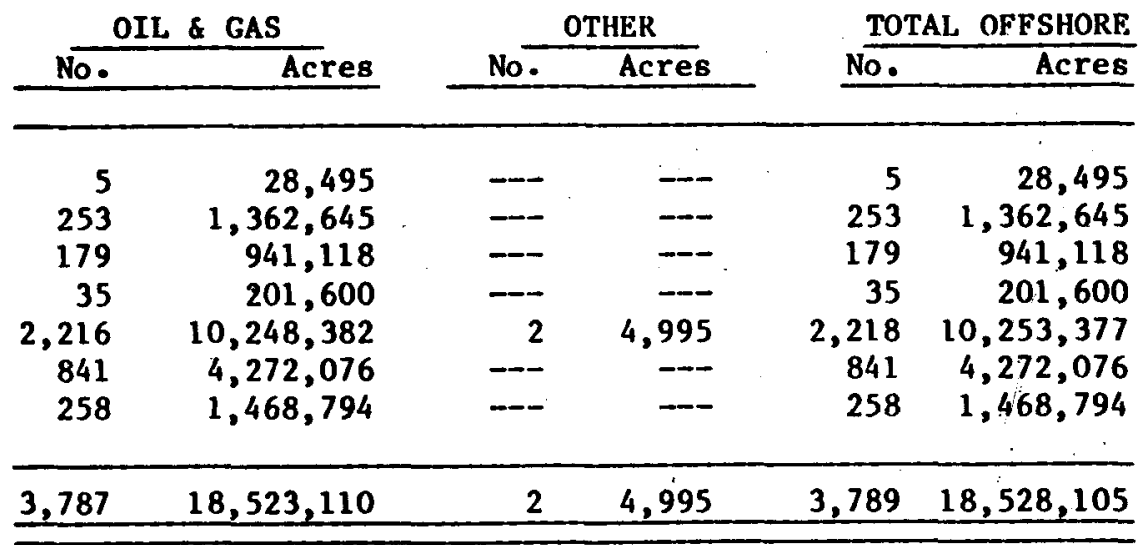


Table 23:

SUMRART OF OIL G GAS AND OTHER MINERAL LAASES, CONTRACTS, LICENSES, AND PERMITS UNDER THE SUPERVISION OF

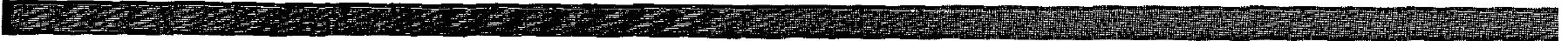

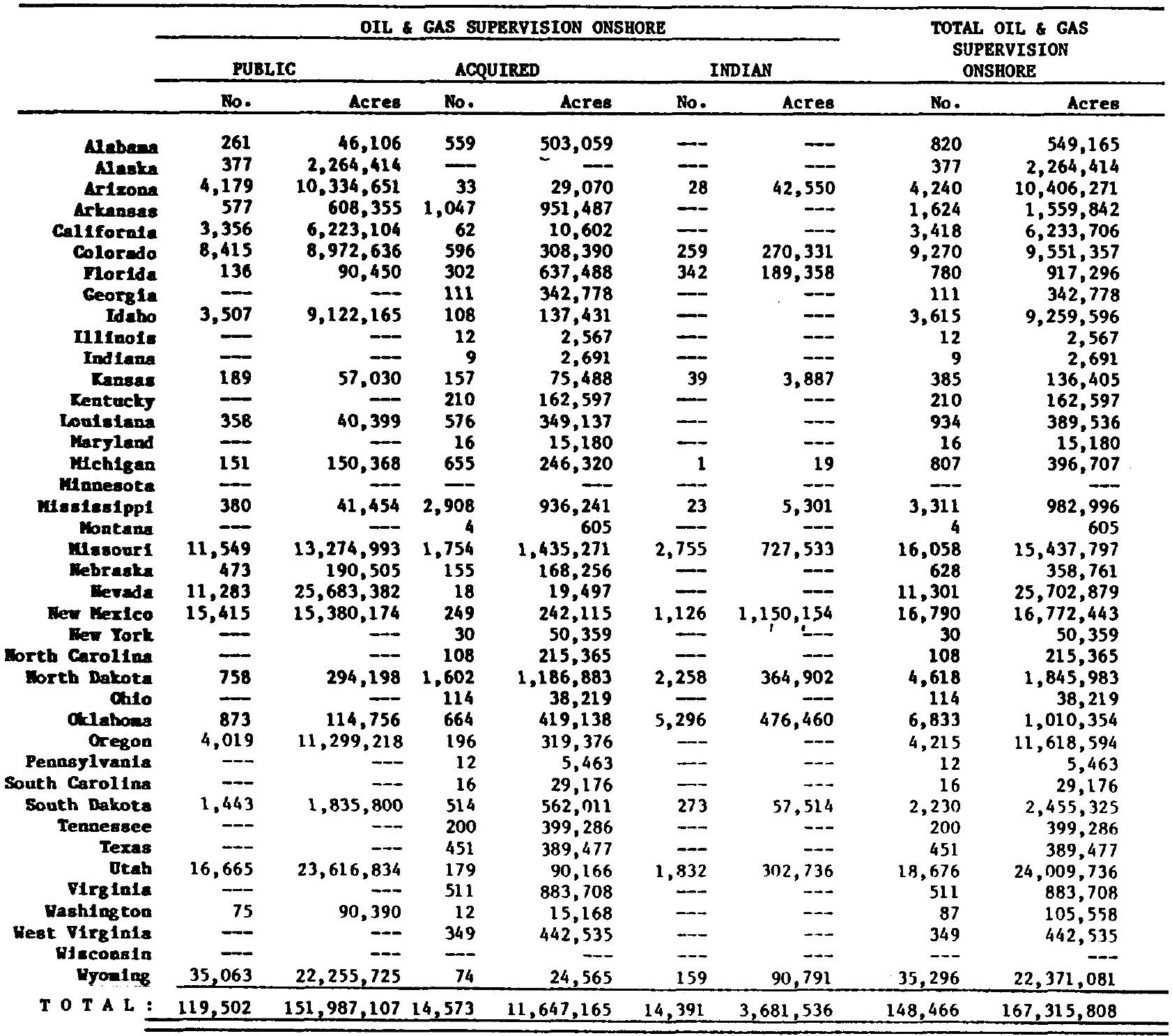

Sourcz: Data provided by the Bureau of Land Management. 
TE DEPARTMENT OF IHE IATERIOR IN FEDERAL AND IKDUAN OASHORE LANDS, AS OP DEcEGBER 31,1983

\begin{tabular}{|c|c|c|c|c|c|c|c|c|c|c|}
\hline \multicolumn{2}{|c|}{ PUBLIC } & \multicolumn{2}{|c|}{ ACQUIRED } & \multicolumn{2}{|c|}{ INDIAN } & \multicolumn{2}{|c|}{$\begin{array}{c}\text { TOTAL "OTHER" } \\
\text { SUPERVISION } \\
\text { ONSHORE }\end{array}$} & \multicolumn{2}{|c|}{$\begin{array}{l}\text { TOTAL FLUID \& SOLID } \\
\text { MINERALS UNDER } \\
\text { SUPERVISION ONSHORE } \\
\end{array}$} & \\
\hline No. & Acres & No. & Acres & No. & Acres & No. & Acres & No. & Acres & \\
\hline 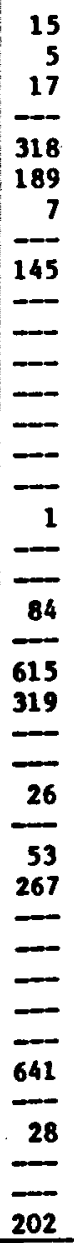 & 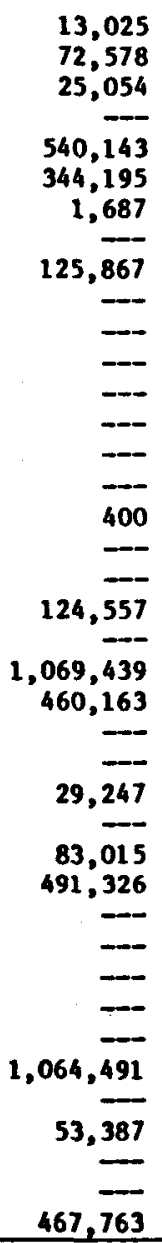 & 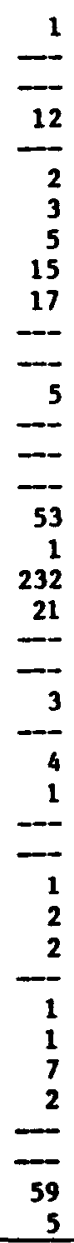 & 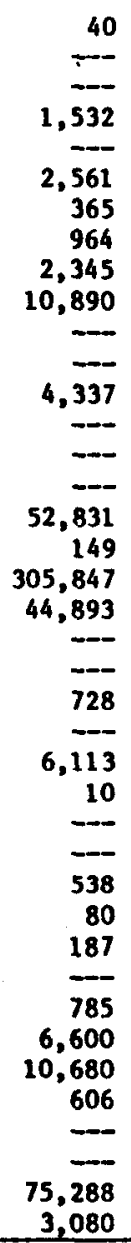 & 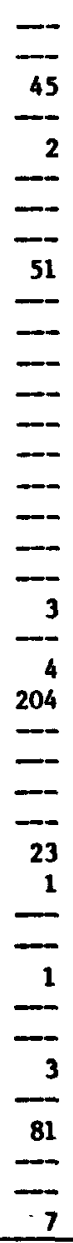 & 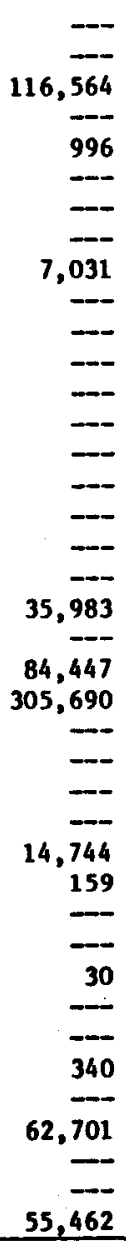 & 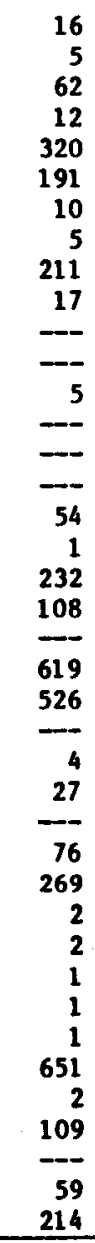 & $\begin{array}{r}13,065 \\
72,578 \\
141,618 \\
1,532 \\
541,139 \\
346,756 \\
2,052 \\
964 \\
135,243 \\
10,890 \\
- \\
- \\
4,337 \\
- \\
- \\
- \\
53,231 \\
149 \\
305,847 \\
205,433 \\
- \\
1,153,886 \\
766,581 \\
6,113 \\
29,257 \\
-79 \\
97,759 \\
492,023 \\
80 \\
187 \\
30 \\
7,685 \\
675,511 \\
606 \\
116,088 \\
75,288 \\
526,305 \\
\end{array}$ & $\begin{array}{r}836 \\
382 \\
4,302 \\
1,636 \\
3,738 \\
9,461 \\
790 \\
116 \\
3,826 \\
29 \\
9 \\
385 \\
215 \\
934 \\
16 \\
807 \\
54 \\
3,312 \\
236 \\
16,166 \\
628 \\
11,920 \\
17,316 \\
30 \\
112 \\
4,645 \\
114 \\
6,909 \\
4,484 \\
14 \\
18 \\
2,231 \\
201 \\
452 \\
19,327 \\
513 \\
196 \\
349 \\
59 \\
35,510 \\
\end{array}$ & $\begin{array}{r}562,230 \\
2,336,992 \\
10,547,889 \\
1,561,374 \\
6,774,845 \\
9,898,113 \\
919,348 \\
343,742 \\
9,394,839 \\
13,457 \\
2,691 \\
136,405 \\
166,934 \\
389,536 \\
15,180 \\
396,707 \\
53,231 \\
983,145 \\
306,452 \\
15,643,230 \\
358,761 \\
26,856,765 \\
17,539,024 \\
50,359 \\
221,478 \\
1,875,240 \\
38,219 \\
1,108,113 \\
12,110,617 \\
5,543 \\
29,363 \\
2,455,355 \\
400,071 \\
396,077 \\
25,085,247 \\
884,314 \\
221,646 \\
442,535 \\
75,288 \\
22,897,386 \\
\end{array}$ & 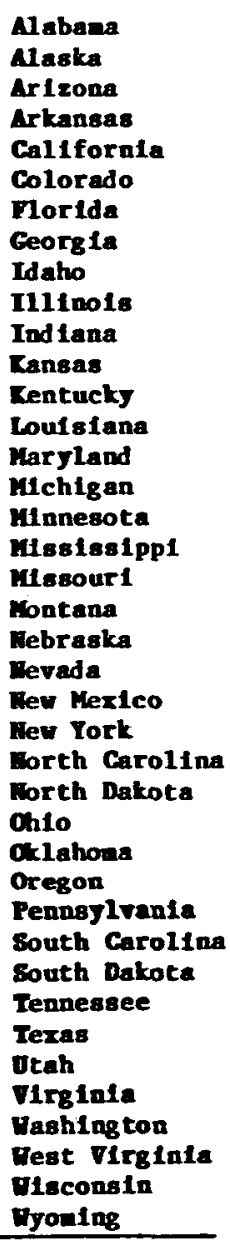 \\
\hline 932 & $4,966,337$ & 455 & 531,449 & 425 & 684,147 & 3,812 & $6,181,933$ & 152,278 & $173,497,741$ & \\
\hline
\end{tabular}


Table 24:

SUNALRT OF GBNERAL TERAS IN FEDERAL AND INDLAN MINERAL LEASES

\begin{tabular}{|c|c|c|c|c|c|}
\hline & \multicolumn{5}{|c|}{ OIL \& GAS LRASES / FEDERAL ONSHORE LAMDS } \\
\hline & $\begin{array}{l}\text { Customary } \\
\text { Royalty Rate }\end{array}$ & $\begin{array}{r}\text { Annual Rental } \\
\& \text { Other Fees } \\
\end{array}$ & $\begin{array}{l}\text { Duration } \\
\text { of Lease }\end{array}$ & $\begin{array}{l}\text { Size of } \\
\text { Lease } \\
\end{array}$ & $\begin{array}{l}\text { Bond ing } \\
\text { Requirements }\end{array}$ \\
\hline $\begin{array}{l}\text { Ton-Competitive } \\
\text { Lesses: }\end{array}$ & $121 / 2 \%$ on production & $\begin{array}{l}\$ 1 \text { per acre or } \\
\$ 2 \text { per acre lf known } \\
\text { geologic structure. } \\
\$ 3 \text { per acre for } 6 \text { to } 10 \\
\text { years for simultaneous } \\
\text { leasing; } \$ 1 \text { per acre } \\
\text { minimum royalty after } \\
\text { discovery; } \$ 75 \text { filing } \\
\text { fee per lease }\end{array}$ & $\begin{array}{l}10 \text { years \& as } \\
\text { long as capable } \\
\text { of producing } \\
\text { in paying } \\
\text { quantities, } \\
\text { etc. }\end{array}$ & $\begin{array}{l}10,240 \text { acres maximum } \\
\text { within } 6 \text {-mile square, } \\
\text { or } 640 \text { acres minimum }\end{array}$ & $\begin{array}{l}\text { Lease } \$ 10,000 \\
\text { State } \$ 25,000 \\
\text { Nat1onw1de } \$ 150,000\end{array}$ \\
\hline $\begin{array}{l}\text { Competitive } \\
\text { Leases: }\end{array}$ & $\begin{array}{l}\text { O11 } 121 / 2 \% \text { to } 25 \% \& \\
\text { Gas } 121 / 2 \% \text { or } 162 / 3 \% \text {, } \\
\text { depending on production } \\
\text { per well per day for } \\
\text { the calendar month }\end{array}$ & $\begin{array}{l}\$ 2 \text { per acre; } \$ 1 \text { per } \\
\text { acre minimum royalty } \\
\text { after discovery }\end{array}$ & $\begin{array}{l}5 \text { years as } \\
\text { long as capable } \\
\text { of producing } \\
\text { in paying } \\
\text { quantities, etc. }\end{array}$ & 640 acres or less & $\begin{array}{l}\text { Lease } \$ 10,000 \\
\text { State } \$ 25,000 \\
\text { Nationwide } \$ 150,000\end{array}$ \\
\hline \multirow[t]{3}{*}{ *IPRA/Alaska: } & $\begin{array}{l}\text { Not set by law--now } \\
162 / 3 \%\end{array}$ & $\$ 3$ per acre & $\begin{array}{l}10 \text { years (or } \\
\text { less) }\end{array}$ & Up to 60,000 acres & $\begin{array}{l}\text { Corporate Surety- } \\
\$ 100,000\end{array}$ \\
\hline & \multicolumn{5}{|c|}{ OIL \& CAS LEASES / FEDLRAL OFTSHORE LANIOS } \\
\hline & $\begin{array}{c}\text { Customary } \\
\text { Royelty Rate }\end{array}$ & $\begin{array}{r}\text { Annual Rental } \\
\& \text { Other Fees } \\
\end{array}$ & $\begin{array}{l}\text { Duration } \\
\text { of Lease } \\
\end{array}$ & $\begin{array}{l}\text { Stze of } \\
\text { Lease }\end{array}$ & $\begin{array}{c}\text { Bond ing } \\
\text { Requirements } \\
\end{array}$ \\
\hline OCS Leases: & $\begin{array}{l}162 / 3 \% \text { on production, } \\
\text { net profit sharing, } \\
\text { s11ding scale from } \\
121 / 2 \% \text { to } 65 \%, 8 \\
\text { flat rates of } 331 / 3 \% \\
\text { \& above, } 20 \% \text { set-aside } \\
\text { to be offered to small } \\
\text { lndependent refiners }\end{array}$ & $\begin{array}{l}\$ 3 \text { per acre; } \$ 3 \text { per } \\
\text { acre minimum royalty } \\
\text { after discovery; } \\
\$ 10 \text { per acre for } \\
\text { drainage sales; } \\
\$ 10 \text { per acre minimum } \\
\text { royalty for drainage } \\
\text { sales }\end{array}$ & $\begin{array}{l}5 \text { years (not to } \\
\text { exceed } 10 \text { years) } \\
\text { \& as long as } \\
\text { producing in } \\
\text { paying quantities, } \\
\text { etc. }\end{array}$ & $\begin{array}{l}5,760 \text { acres unless } \\
\text { a larger area } 1 \mathrm{~s} \\
\text { needed for a } \\
\text { production untt }\end{array}$ & $\begin{array}{l}\text { Lease } \$ 50,000 \\
\text { Area } \$ 300,000\end{array}$ \\
\hline
\end{tabular}


Table 24 (cont'd):

SUMAART OF GENERAL TERMS IN FEDERAL AND IMDIAN MIMERAL LRASES

OIL \& GAS LEASES / INDIAN LANDS

\begin{tabular}{|c|c|c|c|c|c|}
\hline & $\begin{array}{c}\text { Customary } \\
\text { Royalty Rate } \\
\end{array}$ & $\begin{array}{r}\text { Annual Rental } \\
\& \text { Other Fees } \\
\end{array}$ & $\begin{array}{l}\text { Duration } \\
\text { of Lease } \\
\end{array}$ & $\begin{array}{c}\text { S1ze of } \\
\text { Lease }\end{array}$ & $\begin{array}{c}\text { Bond ing } \\
\text { Requirements }\end{array}$ \\
\hline Ind Ian Leases: & $\begin{array}{l}121 / 2 \% \text { on production } \\
\text { or higher; some provide } \\
162 / 3 \% \text { on production } \\
\text { (current average } 14 \% \text { ) }\end{array}$ & $\begin{array}{l}\$ 1.25 \text { per acre } \\
\text { credited against } \\
\text { royalty payments for } \\
\text { the lease year; } \$ 10 \\
\text { filing fee for each } \\
\text { lease or each transfer } \\
\text { or assignment of a lease }\end{array}$ & $\begin{array}{l}10 . \text { years \& as } \\
\text { long as still } \\
\text { producing in } \\
\text { paying quantities, } \\
\text { etc. }\end{array}$ & 2,560 acres or less & $\begin{array}{l}\text { Lease } \$ 500 \text { to } \$ 2,000 \text {, } \\
\text { depending on acreage } \\
\text { Nationwide } \$ 75,000\end{array}$ \\
\hline
\end{tabular}

COAL IRASES / TEDERAL LANDS

\begin{tabular}{|c|c|c|c|c|c|}
\hline & $\begin{array}{c}\text { Customary } \\
\text { Royalty Rate }\end{array}$ & $\begin{array}{c}\text { Annual Rental } \\
\text { \& Other Fees }\end{array}$ & $\begin{array}{l}\text { Duration } \\
\text { of Lease }\end{array}$ & $\begin{array}{c}\text { Size of } \\
\text { Lease }\end{array}$ & $\begin{array}{c}\text { Bond Ing } \\
\text { Requirements } \\
\end{array}$ \\
\hline $\begin{array}{l}\text { Jeases sold } \\
\text { before } \\
\text { August 1976: }\end{array}$ & $\begin{array}{l}151 / 2 \& \text { ton under- } \\
\text { ground; } 171 / 2 \& \text { ton } \\
\text { surface mines }\end{array}$ & $\begin{array}{l}\$ 1 \text { per acre credited } \\
\text { against royalty pay- } \\
\text { ments for the lease } \\
\text { year }\end{array}$ & $\begin{array}{l}20 \text { years \& } \\
\text { continued if } \\
\text { producing }\end{array}$ & $\begin{array}{l}\text { Not more than } 46,080 \\
\text { acres in one State } \\
\& \text { no more than } \\
100,000 \text { acres in } \\
\text { United States for } \\
\text { one or more leases }\end{array}$ & $\begin{array}{l}\text { Nonproducing Leases: } \\
\$ 5,000 \text { or amount } \\
\text { equal to annual rent } \\
\text { rounded to } \$ 1,000, \\
\text { whichever } 18 \text { greater } \\
\text { Producing Leases: } \\
3 \text { months production } \\
\text { royalty }\end{array}$ \\
\hline $\begin{array}{l}\text { Leases sold } \\
\text { after } \\
\text { August 1976: }\end{array}$ & $\begin{array}{l}\text { Re-ad justed \& new } \\
\text { leases: } 8 \% \text { of value } \\
\text { underground; } 121 / 2 \% \\
\text { of value surface } \\
\text { (current average } 61 \$ \\
\text { ton or } 4.1 \% \text { of value) }\end{array}$ & $\begin{array}{l}\$ 3 \text { per acre not credited } \\
\text { agalnst royalty payments; } \\
\text { advance royalty payments; } \\
\$ 250 \text { lease flling fee, } \\
\$ 50 \text { transfer fee }\end{array}$ & (same as above) & (same as above) & (same as above) \\
\hline
\end{tabular}


Table 24 (cont'd):

SUMART OR GBNERAL TERMS IN FEDERAI AND IMDIAN MINERAL IRASES

\begin{tabular}{|c|c|c|c|c|c|}
\hline & \multicolumn{5}{|c|}{ COAL LFASES / IMDLAN LATDS } \\
\hline & $\begin{array}{c}\text { Customary } \\
\text { Royalty Rate }\end{array}$ & $\begin{array}{r}\text { Annual Rental } \\
\& \text { Other Fees } \\
\end{array}$ & $\begin{array}{l}\text { Duration } \\
\text { of Lease }\end{array}$ & $\begin{array}{c}\text { S1ze of } \\
\text { Lease }\end{array}$ & $\begin{array}{c}\text { Bond 1ng } \\
\text { Requitrements } \\
\end{array}$ \\
\hline Ind Lan Leases: & $\begin{array}{l}\text { Not less than } 10 \notin \text { ton } \\
\text { (current average } 29 \$ \\
\text { ton) }\end{array}$ & $\begin{array}{l}\$ 1 \text { per acre } \$ 10 \text { per } \\
\text { acre development } \\
\text { expenditure }\end{array}$ & $\begin{array}{l}10 \text { years \& } \\
\text { as long as } \\
\text { producing in } \\
\text { paying quanti- } \\
\text { tes, etc. }\end{array}$ & $\begin{array}{l}2,560 \text { acres or more } \\
\text { 1f authorized by } \\
\text { Commissioner }\end{array}$ & $\begin{array}{l}\text { Lease } \$ 500 \text { to } \$ 2,000 \\
\text { or State } \$ 75,000-- \\
\text { depending on acreage; } \\
\text { Nationwide } \$ 75,000 \text {, } \\
\text { or as determined by } \\
\text { the Secretary }\end{array}$ \\
\hline
\end{tabular}

OTHER MINERAL LRASES / FEDERAL LAKDS

\begin{tabular}{|c|c|c|c|c|c|}
\hline & $\begin{array}{c}\text { Customary } \\
\text { Royalty Rate }\end{array}$ & $\begin{array}{r}\text { Annual Rental } \\
\& \text { Other Fees } \\
\end{array}$ & $\begin{array}{l}\text { Duration } \\
\text { of Lease }\end{array}$ & $\begin{array}{l}\text { S1ze of } \\
\text { Lease }\end{array}$ & $\begin{array}{c}\text { Bond Ing } \\
\text { Requitrements } \\
\end{array}$ \\
\hline $\begin{array}{l}\text { Potaseiun, } \\
\text { Sodiun : } \\
\text { Fhosphate } \\
\text { Leases: }\end{array}$ & Average $5 \%$ of value & $\begin{array}{l}25 \$ \text { first year; } \\
50 \$ \text { second year } \\
\$ 1 \text { per acre there- } \\
\text { after, credited } \\
\text { against royalties; } \\
\$ 2 \text { per acre min1mum } \\
\text { royalty indexed } \\
\text { annually with BLS } \\
\text { commodity Index }\end{array}$ & $\begin{array}{l}20 \text { years, } \\
\text { sub ject to } \\
\text { read jus tment }\end{array}$ & 2,560 acres & $\begin{array}{l}\text { Not less than } \$ 5,000 \\
\text { State } \$ 25,000 \\
\text { Nationwide } \$ 75,000\end{array}$ \\
\hline $\begin{array}{l}\text { Sulfur } \\
\text { Leases: }\end{array}$ & $\begin{array}{l}\text { Not less than } 5 \% \text { of } \\
\text { value }\end{array}$ & $\begin{array}{l}50 \text { per acre credited } \\
\text { against royalties; } \\
\$ 2 \text { per acre minimum } \\
\text { royalty indexed } \\
\text { annually with BLs } \\
\text { commodity index }\end{array}$ & 20 years & 640 acres & Not less than $\$ 5,000$ \\
\hline $\begin{array}{l}\text { Asphalt } \\
\text { Leases: }\end{array}$ & Per lease terms & $50 \&$ per acre & 10 years & 2,560 acres & As determined \\
\hline $\begin{array}{l}\text { lead, zinc, } \\
\text { Iron \& other } \\
\text { colid intineral } \\
\text { Leases: }\end{array}$ & Average $5 \%$ of value & $\begin{array}{l}\$ 1 \text { per acre credited } \\
\text { against royalties } \\
\text { (up to } \$ 10 \text { if develop- } \\
\text { ment contract) }\end{array}$ & $\begin{array}{l}20 \text { years } \\
\text { (renewals } 10 \\
\text { years) }\end{array}$ & 2,560 acres & Not less than $\$ 5,000$ \\
\hline
\end{tabular}


Table 24 (cont'd):

SUMMARY OF GENERAL TERMS IN FEDERAL ATD IMDIAN MINERAL LEASES

\begin{tabular}{|c|c|c|c|c|c|}
\hline $\begin{array}{l}\text { O11 shale } \\
\text { G1lsonite } \\
\text { Leases: }\end{array}$ & $\begin{array}{l}\text { Per lease terms } \\
\text { (o11 shale--average } \\
12 \phi \text { ton; gilsonite } \\
12.5 \% \text { of value) }\end{array}$ & Per lease terms & Per lease & Per lease & Per lease \\
\hline $\begin{array}{l}\text { Ceothernal } \\
\text { Leases: }\end{array}$ & $\begin{array}{l}10 \% \text { to } 15 \% \text { of value } \\
\text { of steam \& not less } \\
\text { than } 5 \% \text { of value of } \\
\text { demineralized water; } \\
\text { "By-products" royalty } \\
\text { rate* determined by } \\
\text { particular mineral } \\
\text { (generally } \dot{5} \%)\end{array}$ & $\begin{array}{l}\text { \$1 per acre; } \$ 2 \text { per } \\
\text { acre for KGRA }\end{array}$ & $\begin{array}{l}10 \text { years \& as } \\
\text { long as capable } \\
\text { of producing } \\
\text { in paying } \\
\text { quantities }\end{array}$ & $\begin{array}{l}2,560 \text { acres \& not } \\
\text { less } 640 \text { acres--can } \\
\text { be less than } 640 \\
\text { for nonelectrical } \\
\text { uses }\end{array}$ & $\begin{array}{l}\text { Lease } \$ 10,000 \\
\text { State } \$ 50,000 \\
\text { Nationw1de } \$ 150,000 \\
\text { Protect1on of } \\
\text { surface owner-- } \\
\$ 5,000, \text { minimum }\end{array}$ \\
\hline
\end{tabular}

OTHER MINERAL IEASES / IEDIAT LANDS

\begin{tabular}{|c|c|c|c|c|c|}
\hline & $\begin{array}{c}\text { Customary } \\
\text { Royalty Rate }\end{array}$ & $\begin{array}{r}\text { Annual Rental } \\
\& \text { Other Fees } \\
\end{array}$ & $\begin{array}{l}\text { Duration } \\
\text { of Lease } \\
\end{array}$ & $\begin{array}{c}\text { Size of } \\
\text { Lease }\end{array}$ & $\begin{array}{c}\text { Bond 1ng } \\
\text { Requitrements } \\
\end{array}$ \\
\hline $\begin{array}{l}\text { Copper, Lead, } \\
\text { Zinc, } \\
\text { Tungaten } \\
\text { Leases: }\end{array}$ & $\begin{array}{l}\text { Not less than } 10 \% \\
\text { of value }\end{array}$ & $\begin{array}{l}\$ 1 \text { per acre \& } \$ 10 \text { per } \\
\text { acre developuent } \\
\text { expend } 1 \text { ture (varles } \\
\text { greatly) }\end{array}$ & $\begin{array}{l}10 \text { years a as } \\
\text { long thereafter } \\
\text { as producing in } \\
\text { paying quantities, } \\
\text { etc. }\end{array}$ & 2,560 acres & $\begin{array}{l}\text { Lease-as determined } \\
\text { or State } \$ 15,000 \\
\text { Nat1onwide } \$ 75,000\end{array}$ \\
\hline $\begin{array}{l}\text { Gold silver } \\
\text { Leases: }\end{array}$ & $\begin{array}{l}\text { Not less than } 10 \% \text { of } \\
\text { value of bullion per } \\
\text { mint returns }\end{array}$ & (same as above) & 10 years & 2,560 acres & (same as above) \\
\hline $\begin{array}{l}\text { Leases for } \\
\text { other ores } \\
\text { metals: }\end{array}$ & $\begin{array}{l}\text { Not less than } 10 \% \text { of } \\
\text { value }\end{array}$ & (same as above) & 10 years & 2,560 acres & (same as above) \\
\hline
\end{tabular}

Note: Lease terms may be extended for various reasons as authorized by the regulations.

A $\$ 10$ fee 18 separately required for flling, transferring, or assigning an Indian

lease; the same fee is also required for certain other Federal lease commodities. 
Table 25:

SUMMARY OF OIL, CAS, AND COAL PRODOCTION FROM FEDERAL AND INDIAN LEASES AS A PERCENTAGe OF TOTAL U.S. PRODUCTION OF OIL, GAS, AND COAL, 1950-83

\begin{tabular}{|c|c|c|c|}
\hline & $\begin{array}{c}\text { orl In } \\
\text { Total } \\
\text { U.s. Volume }\end{array}$ & $\begin{array}{l}\text { LRRELS } \\
\text { Federal } \\
\& \text { Indian }\end{array}$ & $\begin{array}{c}\text { PERCENTAGE } \\
\text { of } \\
\text { U.S. TOTAL } \\
\end{array}$ \\
\hline \multirow[t]{2}{*}{$\begin{array}{r}1950-79 \\
1980 \\
1981 \\
1982 \\
1983 \\
1950-83\end{array}$} & $\begin{array}{r}85,453 \\
3,138 \\
3,125 \\
3,157 \\
3,159 \\
98,032 \\
\end{array}$ & $\begin{array}{r}11,018 \\
449 \\
467 \\
491 \\
511 \\
12,936 \\
\end{array}$ & $\begin{array}{l}12.9 x \\
14.3 \\
14.9 \\
15.6 \\
16.2 \\
13.2 \\
\end{array}$ \\
\hline & $\begin{array}{c}\text { Cas } \\
\text { Total } \\
\text { U.S Volume } \\
\end{array}$ & $\begin{array}{l}\text { HCF } \\
\text { Federal } \\
\& \text { Indian }\end{array}$ & $\begin{array}{c}\text { PERCENTAGE } \\
\text { of } \\
\text { U.S. TOTAL }\end{array}$ \\
\hline \multirow[t]{2}{*}{$\begin{array}{r}1950-79 \\
1980 \\
1981 \\
1982 \\
1983 \\
1950-83\end{array}$} & $\begin{array}{r}464,904 \\
20,180 \\
19,956 \\
18,520 \\
16,474 \\
540,034 \\
\end{array}$ & $\begin{array}{r}68,410 \\
5,788 \\
6,052 \\
5,974 \\
5,044 \\
91,268 \\
\end{array}$ & $\begin{array}{l}14.77 \\
28.7 \\
30.3 \\
32.2 \\
30.6 \\
16.9 \\
\end{array}$ \\
\hline & $\begin{array}{c}\text { COAL } \\
\text { Total } \\
\text { U.S. Volume }\end{array}$ & $\begin{array}{l}\text { TOxS } \\
\text { Federal } \\
\text { \& Ind Ian }\end{array}$ & $\begin{array}{c}\text { PERCENTAGE } \\
\text { of } \\
\text { U.S. TOTAL }\end{array}$ \\
\hline $\begin{array}{r}1950-79 \\
1980 \\
1981 \\
1982 \\
1983 \\
1950-83\end{array}$ & $\begin{array}{r}14,036 \\
830 \\
824 \\
838 \\
785 \\
17,313 \\
\end{array}$ & $\begin{array}{r}556 \\
93 \\
139 \\
130 \\
134 \\
1,052 \\
\end{array}$ & $\begin{array}{c}4.07 \\
11.2 \\
16.9 \\
15.5 \\
17.1 \\
6.1 \\
\end{array}$ \\
\hline
\end{tabular}

HOTE: 011 volume in millions of barrels (42 U.S. gallons) includes crude oll and lease condensate; natural gas in millions of Mcf (thousand cublc feet); and coal In millions of short tons ( $* 2,000)$ includes anthracite, bituminous coal, and IIgnite. Data by calendar year are rounded.

SOURCE: Monthly Energy Revlew, Department of Energy, for U.S. totals; certaln other data are estimated in part by MS. 